\title{
Room-temperature Formal Aza-Wacker Cyclization through Synergistic Copper/TEMPO-catalyzed Radical Relay
}

Dong Yang, ${ }^{\dagger}$ Jiaming Chen,$^{\dagger}$ Yanping Huang, ${ }^{\dagger},+$ Huiquan Pan,${ }^{\dagger}$ Jingqi Shi, ${ }^{\dagger}$ Yingyue Zhang, ${ }^{\dagger}$ Fang Wang, ${ }^{\dagger}$ and Ziyuan $\mathrm{Li}^{*}{ }^{\dagger}$

${ }^{\dagger}$ Department of Pharmaceutical and Biological Engineering, School of Chemical Engineering, Sichuan University, No.24 South Section 1, Yihuan Road, Chengdu 610065, China

${ }^{*}$ Engineering Experimental Teaching Centre, School of Chemical Engineering, Sichuan University, No.24 South Section 1, Yihuan Road, Chengdu 610065, China

E-mail: liziyuan@scu.edu.cn; Fax: (+86)28-85405221

Table of Contents

\begin{tabular}{|c|c|}
\hline General Remarks & S2 \\
\hline Preparation of Substrates & S3-S9 \\
\hline Detailed Results of Reaction Condition Screening & S10-S11 \\
\hline Experimental Procedure and Characterization Data & S12-S19 \\
\hline Kinetic Studies & S20-S23 \\
\hline Control Experiments & S24-S26 \\
\hline Gram-scale Synthesis & S27 \\
\hline References & S28 \\
\hline${ }^{1} \mathrm{H}$ NMR and ${ }^{13} \mathrm{C}$ NMR Spectra of Products & S29-S78 \\
\hline
\end{tabular}




\section{General Remarks}

All commercially available compounds were purchased from Sigma-Aldrich, Alfa-Aesar, Acros, J\&K Chemicals, Adamas-beta, Accela ChemBio and Aladdin Chemicals. $\mathrm{CuCl}$ was purchased from Acros (99.99\% extra pure, CAS No. 7758-89-6). TEMPO (98\%, CAS No. 2564-83-2), NFSI (97\%, CAS No. 133745-75-2) and $\mathrm{MeCN}$ (99.9\%, SafeDry, water $<50 \mathrm{ppm}$ ) were purchased from Adamas. Bromotrichloromethane (97\%, CAS No. 75-62-7) were purchased from TCI. Unless otherwise noted, materials obtained from commercial suppliers were used without further purification. Carbamate substrates were prepared via condensation between corresponding isocyanates and alcohols according to literature reported methods. ${ }^{[1]}$ Amide substrates were preprared through literature reported routes starting from 2-carboxybenzaldehyde, ${ }^{[1]}$ or from 4-bromobutyric Acid. Products were purified by flash chromatography on silica gel using petroleum ether, ethyl acetate and dichloromethane as the eluents. ${ }^{1} \mathrm{H}-\mathrm{NMR}$ spectra were recorded on Bruker AVANCE III-400 or JNM-ECZ400S/L1 spectrometers. Chemical shifts (in ppm) were referenced with TMS in $\mathrm{CDCl}_{3}(0 \mathrm{ppm}) .{ }^{13} \mathrm{C}-\mathrm{NMR}$ spectra were obtained by using the same NMR spectrometers and were calibrated with $\mathrm{CDCl}_{3}(\delta=77.00 \mathrm{ppm})$ or DMSO- $d_{6}(\delta=39.50 \mathrm{ppm})$. High resolution mass spectra were obtained from an Agilent 6520B Q-TOF mass spectrometer with electron spray ionization (ESI) as the ion source. 


\section{Preparation of Substrates}

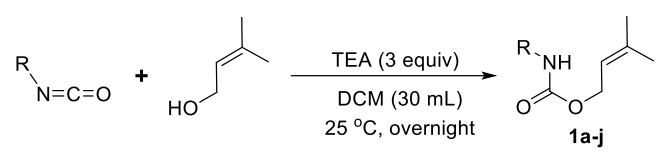

Typical Procedure: To a $100 \mathrm{~mL}$ flask charged with a solution of corresponding isocyanate $(10 \mathrm{mmol})$ and triethylamine $(30 \mathrm{mmol})$ in dichloromethane $(20 \mathrm{~mL})$ was added dropwise a solution of 3-methyl-2-buten-1-ol $(10 \mathrm{mmol})$ in dichloromethane $(10 \mathrm{~mL})$. The reaction mixture was stirred at $25{ }^{\circ} \mathrm{C}$ overnight. After quenched with saturated $\mathrm{NH}_{4} \mathrm{Cl}$ (aq.), the mixture was washed with water, dried over $\mathrm{Na}_{2} \mathrm{SO}_{4}$, and concentrated in vacuo to give dark residue, which was then purified by flash chromatography using petroleum ether and ethyl acetate $(10: 1, v / v)$ as the eluent on silical gel to afford corresponding carbamate substrates 1a-j.

\section{3-Methylbut-2-en-1-yl ethylcarbamate (1a)}

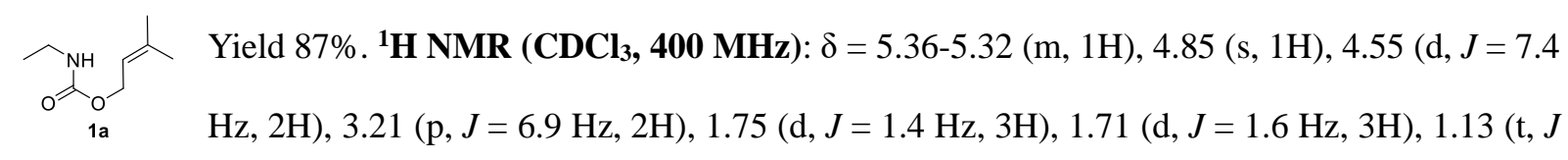
$=7.2 \mathrm{~Hz}, 1 \mathrm{H}) \mathrm{ppm}$.

\section{3-Methylbut-2-en-1-yl $p$-tolylcarbamate (1b)}

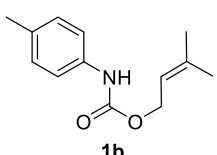

Yield 77\%. ${ }^{1} \mathbf{H}$ NMR $\left(\mathbf{C D C l}_{3}, \mathbf{4 0 0} \mathbf{M H z}\right): \delta=7.25(\mathrm{~d}, J=8.0 \mathrm{~Hz}, 2 \mathrm{H}), 7.09(\mathrm{~d}, J=7.9 \mathrm{~Hz}$, 2H), $6.61(\mathrm{~s}, 1 \mathrm{H}), 5.42-5.36(\mathrm{~m}, 1 \mathrm{H}), 4.65(\mathrm{~d}, J=7.0 \mathrm{~Hz}, 2 \mathrm{H}), 2.29(\mathrm{~s}, 1 \mathrm{H}), 1.77(\mathrm{~d}, J=$ $1.3 \mathrm{~Hz}, 3 \mathrm{H}), 1.74(\mathrm{~d}, J=1.4 \mathrm{~Hz}, 3 \mathrm{H}) \mathrm{ppm}$.

\section{3-Methylbut-2-en-1-yl (4-chlorophenyl)carbamate (1c)}

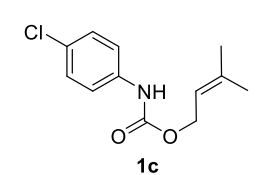

Yield 81\%. ${ }^{\mathbf{1}} \mathbf{H}$ NMR $\left(\mathbf{C D C l}_{3}, \mathbf{4 0 0} \mathbf{M H z}\right): \delta=7.32(\mathrm{~d}, J=8.6 \mathrm{~Hz}, 2 \mathrm{H}), 7.24(\mathrm{~d}, J=8.8$ $\mathrm{Hz}, 2 \mathrm{H}), 6.69(\mathrm{~s}, 1 \mathrm{H}), 5.40-5.36(\mathrm{~m}, 1 \mathrm{H}), 4.66(\mathrm{~d}, J=7.2 \mathrm{~Hz}, 2 \mathrm{H}), 1.77(\mathrm{~d}, J=1.5 \mathrm{~Hz}$, 3H), $1.74(\mathrm{~d}, J=1.4 \mathrm{~Hz}, 3 \mathrm{H}) \mathrm{ppm}$.

\section{3-Methylbut-2-en-1-yl (4-bromophenyl)carbamate (1d)}

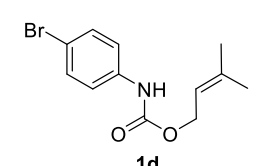

Yield 76\%. ${ }^{1} \mathbf{H}$ NMR $\left(\mathbf{C D C l}_{3}, \mathbf{4 0 0} \mathbf{M H z}\right): \delta=7.40(\mathrm{~d}, J=8.4 \mathrm{~Hz}, 2 \mathrm{H}), 7.27(\mathrm{~d}, J=8.2$ $\mathrm{Hz}, 2 \mathrm{H}), 6.60$ (s, 1H), 5.41-5.36 (m, 1H), 4.66 (d, $J=7.3 \mathrm{~Hz}, 2 \mathrm{H}), 1.77$ (s, 3H), 1.74 (d, $J$ $=1.4 \mathrm{~Hz}, 3 \mathrm{H}) \mathrm{ppm}$. 


\section{3-Methylbut-2-en-1-yl (4-fluorophenyl)carbamate (1e)}

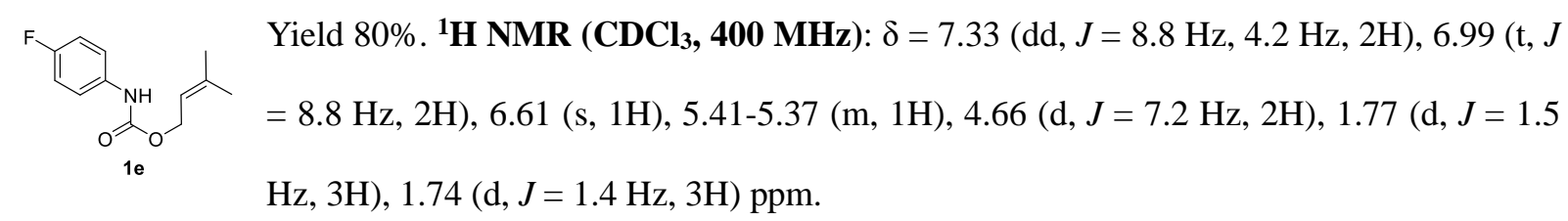

\section{3-Methylbut-2-en-1-yl (4-(trifluoromethyl)phenyl)carbamate (1f)}

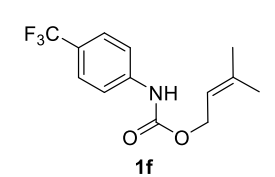

Yield 75\%. ${ }^{1} \mathbf{H}$ NMR $\left(\mathbf{C D C l}_{3}, \mathbf{4 0 0} \mathbf{M H z}\right): \delta=7.55(\mathrm{~d}, J=8.6 \mathrm{~Hz}, 2 \mathrm{H}), 7.49(\mathrm{t}, J=8.6$ Hz, 2H), 6.77 (s, 1H), 5.42-5.37 (m, 1H), 4.68 (d, $J=7.3 \mathrm{~Hz}, 2 \mathrm{H}), 1.78$ (d, $J=1.4 \mathrm{~Hz}$, $3 \mathrm{H}), 1.75(\mathrm{~d}, J=1.4 \mathrm{~Hz}, 3 \mathrm{H}) \mathrm{ppm}$.

3-Methylbut-2-en-1-yl $m$-tolylcarbamate (1g)

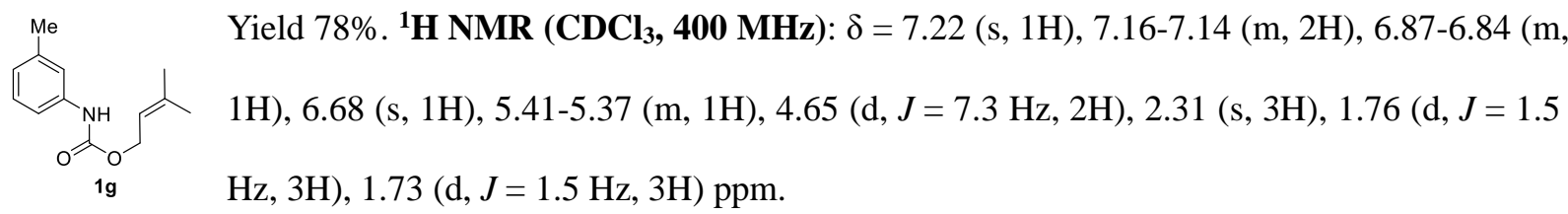

\section{3-Methylbut-2-en-1-yl (3,5-dimethylphenyl)carbamate (1h)}

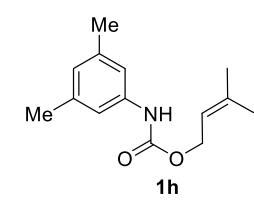

Yield 80\%. ${ }^{1} \mathbf{H}$ NMR $\left(\mathbf{C D C l}_{3}, \mathbf{4 0 0} \mathbf{M H z}\right): \delta=7.00(\mathrm{~s}, 2 \mathrm{H}), 6.68(\mathrm{~d}, J=2.0 \mathrm{~Hz}, 1 \mathrm{H}), 6.63$ (s, 1H), 5.41-5.37 (m, 1H), $4.65(\mathrm{~d}, J=7.2 \mathrm{~Hz}, 2 \mathrm{H}), 2.26(\mathrm{~d}, J=0.4 \mathrm{~Hz}, 6 \mathrm{H}), 1.76(\mathrm{~d}, J=$ $1.5 \mathrm{~Hz}, 3 \mathrm{H}), 1.73(\mathrm{~d}, J=1.5 \mathrm{~Hz}, 3 \mathrm{H}) \mathrm{ppm}$.

\section{3-Methylbut-2-en-1-yl (4-isopropylphenyl)carbamate (1i)}

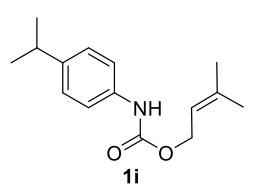

Yield 85\%. ${ }^{1} \mathbf{H}$ NMR $\left(\mathbf{C D C l}_{3}, \mathbf{4 0 0} \mathbf{M H z}\right): \delta=7.28(\mathrm{~d}, J=8.3 \mathrm{~Hz}, 2 \mathrm{H}), 7.13(\mathrm{~d}, J=8.6$ Hz, 1H), 6.76 (s, 1H), 5.41-5.36 (m, 1H), 4.65 (d, $J=7.2 \mathrm{~Hz}, 2 \mathrm{H}), 2.85$ (sept, $J=6.9 \mathrm{~Hz}$, $1 \mathrm{H}), 1.75(\mathrm{~d}, J=1.6 \mathrm{~Hz}, 3 \mathrm{H}), 1.72(\mathrm{~d}, J=1.5 \mathrm{~Hz}, 3 \mathrm{H}), 1.21(\mathrm{~d}, J=6.9 \mathrm{~Hz}, 6 \mathrm{H}) \mathrm{ppm}$.

3-Methylbut-2-en-1-yl (2-methoxyphenyl)carbamate (1j)

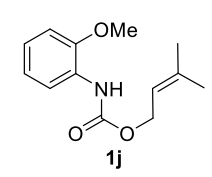

Yield 79\%. ${ }^{1} \mathrm{H}$ NMR $\left(\mathbf{C D C l}_{3}, \mathbf{4 0 0} \mathbf{M H z}\right): \delta=8.09(\mathrm{~d}, J=6.4 \mathrm{~Hz}, 1 \mathrm{H}), 7.25(\mathrm{~s}, 1 \mathrm{H})$, 6.99-6.92 (m, 2H), 6.84-6.82 (m, 1H), 5.43-5.39 (m, 1H), $4.66(\mathrm{~d}, J=7.3 \mathrm{~Hz}, 2 \mathrm{H}), 3.82(\mathrm{~s}$, $3 \mathrm{H}), 1.77(\mathrm{~d}, J=1.4 \mathrm{~Hz}, 3 \mathrm{H}), 1.74(\mathrm{~d}, J=1.5 \mathrm{~Hz}, 3 \mathrm{H}) \mathrm{ppm}$.

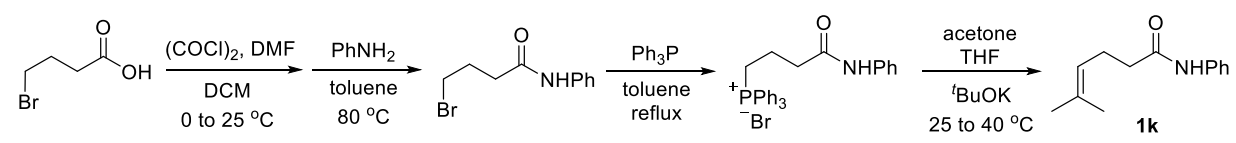

Typical Procedure: To a $100 \mathrm{~mL}$ flask charged with a solution of 4-bromobutyric acid (30 mmol) in dichloromethane $(30 \mathrm{~mL})$ was added dropwise oxalyl chloride $(60 \mathrm{mmol})$ and $2 \sim 3$ drops of DMF at $0{ }^{\circ} \mathrm{C}$. The reaction mixture was stirred at $25{ }^{\circ} \mathrm{C}$ for four hours and concentrated in vacuo to give brown liquid 
residue, which was then added dropwise to another $100 \mathrm{~mL}$ flask charged with a solution of aniline (60 mmol) in toluene $(40 \mathrm{~mL})$ at $0{ }^{\circ} \mathrm{C}$. The reaction mixture was stirred at $0{ }^{\circ} \mathrm{C}$ for 5 minutes and then stirred at $80{ }^{\circ} \mathrm{C}$ overnight. After quenched with $1 \mathrm{M} \mathrm{HCl}$ (aq.), the mixture was washed with water, dried over $\mathrm{Na}_{2} \mathrm{SO}_{4}$, and concentrated in vacuo to give dark residue, which was then purified by flash chromatography using petroleum ether and ethyl acetate $(10: 1, v / v)$ as the eluent on silical gel to afford 4-bromo- $N$-phenylbutanamide in $81 \%$ yield.

Subsequently, to a $100 \mathrm{~mL}$ flash charged with a solution of 4-bromo- $N$-phenylbutanamide $(22 \mathrm{mmol})$ in toluene $(40 \mathrm{~mL})$ was added triphenylphosphine $(44 \mathrm{mmol})$ at $25^{\circ} \mathrm{C}$. The reaction mixture was stirred at 120 ${ }^{\circ} \mathrm{C}$ overnight and cooled to room temperature. The white precipitate was filtered, washed with tert-butyl methyl ether, and dried at $65{ }^{\circ} \mathrm{C}$ in an oven to give the phosphonium in $52 \%$ yield.

Finally, to a $50 \mathrm{~mL}$ flash charged with a solution of the above phosphonium $(6 \mathrm{mmol})$ in THF $(12 \mathrm{~mL})$ was slowly added potassium tert-butoxide $(6 \mathrm{mmol})$. The mixture was stirred at $25{ }^{\circ} \mathrm{C}$ for 2 hours, and a solution of acetone $(24 \mathrm{mmol})$ in THF $(1 \mathrm{~mL})$ was added. Then the reaction mixture was stirred at $40{ }^{\circ} \mathrm{C}$ overnight. After quenched with saturated $\mathrm{NH}_{4} \mathrm{Cl}$ (aq.) and diluted with ethyl acetate, the aqueous layer was extracted with ethyl acetate twice. The combined organic phase was dried over $\mathrm{Na}_{2} \mathrm{SO}_{4}$, and concentrated in vacuo to give dark residue, which was then purified by flash chromatography using petroleum ether and ethyl acetate $(10: 1, v / v)$ as the eluent on silical gel to afford the amide substrate $1 \mathbf{k}$ in $32 \%$ yield.

\section{5-Methyl- $N$-phenylhex-4-enamide (1k)}

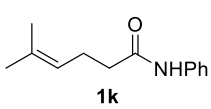

$1 \mathrm{k}$

(s, 3H) ppm.

${ }^{1} \mathrm{H}$ NMR $\left(\mathrm{CDCl}_{3}, 400 \mathrm{MHz}\right): \delta=7.50(\mathrm{~d}, J=7.8 \mathrm{~Hz}, 2 \mathrm{H}), 7.37(\mathrm{~s}, 1 \mathrm{H}), 7.31(\mathrm{t}, J=7.8$ $\mathrm{Hz}, 2 \mathrm{H}), 7.11-7.08(\mathrm{~m}, 1 \mathrm{H}), 5.17(\mathrm{t}, J=6.6 \mathrm{~Hz}, 1 \mathrm{H}), 2.44-2.37(\mathrm{~m}, 4 \mathrm{H}), 1.71(\mathrm{~s}, 3 \mathrm{H}), 1.65$

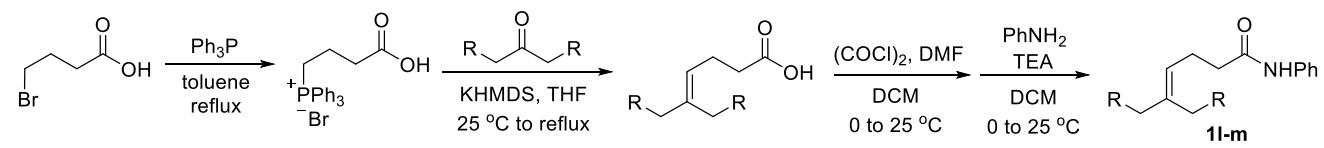

Typical Procedure: To a $100 \mathrm{~mL}$ flask charged with a solution of 4-bromobutyric acid (30 mmol) in toluene $(50 \mathrm{~mL})$ was added triphenylphosphine $(60 \mathrm{mmol})$ at $25^{\circ} \mathrm{C}$. The reaction mixture was stirred at 120 ${ }^{\circ} \mathrm{C}$ overnight and cooled to room temperature. The white precipitate was filtered, washed with tert-butyl methyl ether, and dried at $65{ }^{\circ} \mathrm{C}$ in an oven to give the phosphonium butanoic acid in $79 \%$ yield.

Subsequently, to a $50 \mathrm{~mL}$ flash charged with a solution of the above phosphonium butanoic acid (6 mmol) in THF ( $8 \mathrm{~mL}$ ) was slowly added KHMDS $(12 \mathrm{mmol})$. The mixture was stirred at $25{ }^{\circ} \mathrm{C}$ for 30 minutes, and 
a solution of 3-pentanone or 4-heptanone $(3 \mathrm{mmol})$ in THF $(0.5 \mathrm{~mL})$ was added. Then the reaction mixture was stirred at $70{ }^{\circ} \mathrm{C}$ overnight. After quenched with iced water, $1 \mathrm{M} \mathrm{HCl}$ (aq.) was used to adjust the $\mathrm{pH}$ value to 1.0, and then the mixture was extracted with ethyl acetate for three times. The combined organic phase was dried over $\mathrm{Na}_{2} \mathrm{SO}_{4}$, and concentrated in vacuo to give crude unsaturated acid, which was directly employed in the next step without further purification.

Finally, to a $50 \mathrm{~mL}$ flask charged with a solution of the above unsaturated acid $(3 \mathrm{mmol})$ in dichloromethane $(15 \mathrm{~mL})$ was added dropwise oxalyl chloride $(6 \mathrm{mmol})$ and one drop of DMF at $0{ }^{\circ} \mathrm{C}$. The reaction mixture was stirred at $25^{\circ} \mathrm{C}$ for four hours and concentrated in vacuo to give brown liquid residue, which was then added dropwise to another $50 \mathrm{~mL}$ flask charged with a solution of aniline $(3 \mathrm{mmol})$ and TEA $(6 \mathrm{mmol})$ in dichloromethane $(20 \mathrm{~mL})$ at $0{ }^{\circ} \mathrm{C}$. The reaction mixture was stirred at $0{ }^{\circ} \mathrm{C}$ for 5 minutes and then stirred at $25{ }^{\circ} \mathrm{C}$ overnight. After quenched with $1 \mathrm{M} \mathrm{HCl}$ (aq.), the mixture was diluted with dichloromethane, washed with water, dried over $\mathrm{Na}_{2} \mathrm{SO}_{4}$, and concentrated in vacuo to give dark residue, which was then purified by flash chromatography using petroleum ether and ethyl acetate $(10: 1, v / v)$ as the eluent on silical gel to afford amide $\mathbf{1 l}$ (28\% total yield for 3 steps) or $\mathbf{1 m}$ (23\% total yield for 3 steps) from 3-pentanone or 4-heptanone, respectively.

\section{5-Ethyl- $N$-phenylhept-4-enamide (11)}

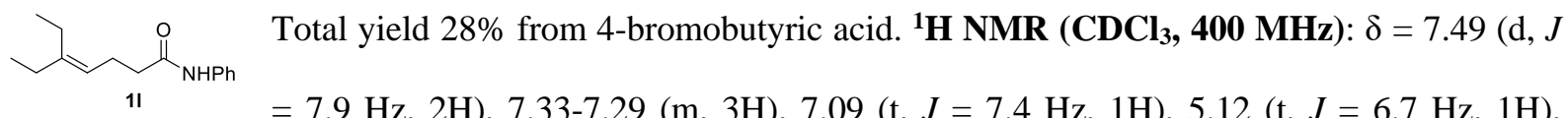

2.45-2.37 (m, 4H), 2.11-2.00 (m, 4H), 1.01-0.95 (m, 6H) ppm.

\section{5-Propyl- $N$-phenyloct-4-enamide (1m)}

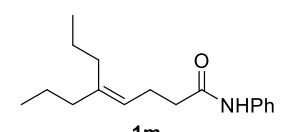

Total yield 23\% from 4-bromobutyric acid. ${ }^{1} \mathrm{H}$ NMR $\left(\mathbf{C D C l}_{3}, 400 \mathrm{MHz}\right): \delta=$

7.52-7.48 (m, 2H), $7.38(\mathrm{~s}, 1 \mathrm{H}), 7.32-7.28(\mathrm{~m}, 2 \mathrm{H}), 7.11-7.07(\mathrm{~m}, 1 \mathrm{H}), 5.16(\mathrm{t}, J=6.7$ $\mathrm{Hz}, 1 \mathrm{H}), 2.45-2.30(\mathrm{~m}, 4 \mathrm{H}), 2.05-1.92(\mathrm{~m}, 4 \mathrm{H}), 1.42-1.35(\mathrm{~m}, 4 \mathrm{H}), 0.89$ (t, $J=7.3 \mathrm{~Hz}, 3 \mathrm{H}), 0.86$ (t, $J=7.4$ $\mathrm{Hz}, 3 \mathrm{H}) \mathrm{ppm}$.

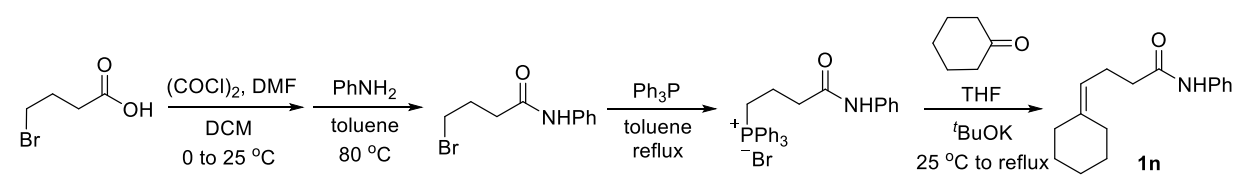

Typical Procedure: The first two steps are identical to the preparation of amide $\mathbf{1 k}$.

The final step: To a $50 \mathrm{~mL}$ flash charged with a solution of the above phosphonium amide (6 mmol) in THF 
$(12 \mathrm{~mL})$ was slowly added potassium tert-butoxide $(6 \mathrm{mmol})$. The mixture was stirred at $25{ }^{\circ} \mathrm{C}$ for 2 hours, and a solution of cyclohexanone $(3 \mathrm{mmol})$ in THF $(1 \mathrm{~mL})$ was added. Then the reaction mixture was stirred at $70{ }^{\circ} \mathrm{C}$ overnight. After quenched with saturated $\mathrm{NH}_{4} \mathrm{Cl}$ (aq.) and diluted with ethyl acetate, the aqueous layer was extracted with ethyl acetate twice. The combined organic phase was dried over $\mathrm{Na}_{2} \mathrm{SO}_{4}$, and concentrated in vасио to give dark residue, which was then purified by flash chromatography using petroleum ether and ethyl acetate $(10: 1, v / v)$ as the eluent on silical gel to afford the amide substrate $1 \mathbf{n}$ in $49 \%$ yield.

\section{4-Cyclohexylidene- $N$-phenylbutanamide (1n)}

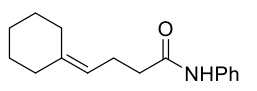

1n

${ }^{1} \mathbf{H}$ NMR $\left(\mathbf{C D C l}_{3}, 400 \mathrm{MHz}\right): \delta=7.60(\mathrm{~s}, 1 \mathrm{H}), 7.50(\mathrm{~d}, J=8.0 \mathrm{~Hz}, 2 \mathrm{H}), 7.29(\mathrm{t}, J=7.8$ $\mathrm{Hz}, 2 \mathrm{H}), 7.08(\mathrm{t}, J=7.4 \mathrm{~Hz}, 1 \mathrm{H}), 5.10(\mathrm{t}, J=6.7 \mathrm{~Hz}, 1 \mathrm{H}), 2.42-2.35(\mathrm{~m}, 4 \mathrm{H}), 2.15-2.05$ (m, 4H), 1.53-1.46 (m, 6H) ppm.

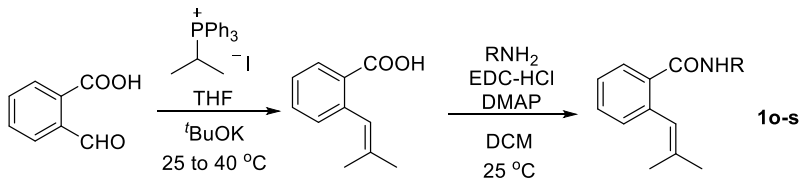

Typical Procedure: To a $100 \mathrm{~mL}$ flask charged with a solution of $i$-propyl triphenylphosphonium iodide (20 mmol) in THF $(20 \mathrm{~mL})$ was added potassium tert-butoxide $(20 \mathrm{mmol})$. The mixture was stirred at $25^{\circ} \mathrm{C}$ for 2 hours, and a solution of 2-carboxybenzaldehyde $(10 \mathrm{mmol})$ in THF $(2 \mathrm{~mL})$ was added. Then the reaction mixture was stirred at $40{ }^{\circ} \mathrm{C}$ overnight. After quenched with $1 \mathrm{M} \mathrm{HCl}$ (aq.) and diluted with ethyl acetate, the aqueous layer was extracted with ethyl acetate twice. The combined organic phase was dried over $\mathrm{Na}_{2} \mathrm{SO}_{4}$, and concentrated in vacuo to give dark residue, which was then purified by flash chromatography using petroleum ether and ethyl acetate $(8: 1, v / v)$ as the eluent on silical gel to afford the 2-alkenylbenzoic acid in $45 \%$ yield.

To a $50 \mathrm{~mL}$ flask charged with a solution of the above 2-alkenylbenzoic acid (3 mmol) and DMAP (7.5 mmol) in DCM $(20 \mathrm{~mL})$ was slowly added $\mathrm{EDC}-\mathrm{HCl}(4.5 \mathrm{mmol})$ at $0{ }^{\circ} \mathrm{C}$. After stirring for 15 minutes, corresponding amines $(4.5 \mathrm{mmol})$ were added, and then the reaction mixture was stirred at $25^{\circ} \mathrm{C}$ overnight. After diluted with DCM, the mixture was washed with saturated $\mathrm{NaHCO}_{3}$ (aq.), $1 \mathrm{M} \mathrm{HCl}$ (aq.), water and brine, dried over $\mathrm{Na}_{2} \mathrm{SO}_{4}$, and concentrated in vacuo to give dark residue, which was then purified by flash chromatography using petroleum ether and ethyl acetate $(10: 1, v / v)$ as the eluent on silical gel to afford corresponding 2-alkenylbenzamide substrates 1o-s. 
2-(2-Methylprop-1-en-1-yl)- $N$-phenylbenzamide (1o)

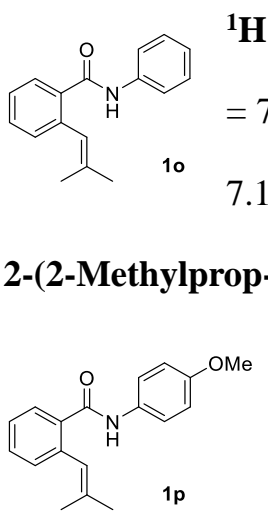

${ }^{1} \mathrm{H}$ NMR $\left(\mathbf{C D C l}_{3}, 400 \mathrm{MHz}\right): \delta=8.04(\mathrm{~s}, 1 \mathrm{H}), 7.90(\mathrm{dd}, J=7.8 \mathrm{~Hz}, 1.5 \mathrm{~Hz}, 1 \mathrm{H}), 7.58(\mathrm{~d}, J$ $=7.9 \mathrm{~Hz}, 2 \mathrm{H}), 7.44(\mathrm{td}, J=7.5 \mathrm{~Hz}, 1.5 \mathrm{~Hz}, 1 \mathrm{H}), 7.38-7.34(\mathrm{~m}, 3 \mathrm{H}), 7.23(\mathrm{~d}, J=7.6 \mathrm{~Hz}, 1 \mathrm{H})$ 7.15-7.11 (m, 1H), $6.53(\mathrm{~s}, 1 \mathrm{H}), 1.99$ (d, $J=1.4 \mathrm{~Hz}, 3 \mathrm{H}), 1.80(\mathrm{~d}, J=1.4 \mathrm{~Hz}, 3 \mathrm{H}) \mathrm{ppm}$.

2-(2-Methylprop-1-en-1-yl)- $N$-(4-methoxyphenyl)benzamide (1p)

${ }^{1} \mathrm{H}_{\mathrm{NMR}}\left(\mathrm{CDCl}_{3}, \mathbf{4 0 0} \mathrm{MHz}\right): \delta=7.95(\mathrm{~s}, 1 \mathrm{H}), 7.87(\mathrm{dd}, J=7.8 \mathrm{~Hz}, 1.6 \mathrm{~Hz}, 1 \mathrm{H}), 7.48$ $(\mathrm{d}, J=9.0 \mathrm{~Hz}, 2 \mathrm{H}), 7.42(\mathrm{td}, J=7.4 \mathrm{~Hz}, 1.5 \mathrm{~Hz}, 1 \mathrm{H}), 7.32(\mathrm{td}, J=7.6 \mathrm{~Hz}, 1.4 \mathrm{~Hz}, 1 \mathrm{H})$, $7.21(\mathrm{~d}, J=7.6 \mathrm{~Hz}, 1 \mathrm{H}), 6.89(\mathrm{~d}, J=9.0 \mathrm{~Hz}, 2 \mathrm{H}), 6.51(\mathrm{~s}, 1 \mathrm{H}), 1.97(\mathrm{~d}, J=1.6 \mathrm{~Hz}, 3 \mathrm{H})$, $1.79(\mathrm{~d}, J=1.4 \mathrm{~Hz}, 3 \mathrm{H}) \mathrm{ppm}$.

\section{2-(2-Methylprop-1-en-1-yl)-N-(4-(tert-butyl)phenyl)benzamide (1q)}

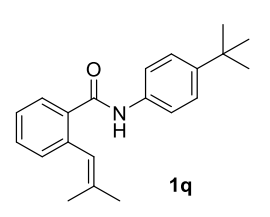

${ }^{1} \mathrm{H}$ NMR $\left(\mathbf{C D C l}_{3}, \mathbf{4 0 0} \mathrm{MHz}\right): \delta=8.09(\mathrm{~s}, 1 \mathrm{H}), 7.82(\mathrm{dd}, J=7.8 \mathrm{~Hz}, 1.5 \mathrm{~Hz}, 1 \mathrm{H}), 7.50$ $(\mathrm{d}, J=8.6 \mathrm{~Hz}, 2 \mathrm{H}), 7.42-7.35(\mathrm{~m}, 3 \mathrm{H}), 7.29(\mathrm{td}, J=7.6 \mathrm{~Hz}, 1.4 \mathrm{~Hz}, 1 \mathrm{H}), 7.20(\mathrm{~d}, J=7.7$ Hz, 1H), $6.49(\mathrm{~s}, 1 \mathrm{H}), 1.96(\mathrm{~d}, J=1.6 \mathrm{~Hz}, 3 \mathrm{H}), 1.77(\mathrm{~d}, J=1.5 \mathrm{~Hz}, 3 \mathrm{H}), 1.31(\mathrm{~s}, 9 \mathrm{H})$ ppm.

\section{2-(2-Methylprop-1-en-1-yl)- $N$-(4-fluorophenyl)benzamide (1r)}

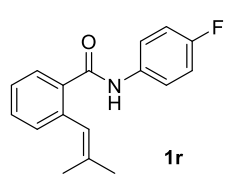

${ }^{1} \mathbf{H}$ NMR $\left(\mathbf{C D C l}_{3}, 400 \mathrm{MHz}\right): \delta=8.02(\mathrm{~s}, 1 \mathrm{H}), 7.89(\mathrm{dd}, J=7.8 \mathrm{~Hz}, 1.5 \mathrm{~Hz}, 1 \mathrm{H}), 7.54$ $(\mathrm{dd}, J=8.9 \mathrm{~Hz}, 4.2 \mathrm{~Hz}, 2 \mathrm{H}), 7.44(\mathrm{td}, J=7.6 \mathrm{~Hz}, 1.5 \mathrm{~Hz}, 1 \mathrm{H}), 7.33(\mathrm{td}, J=7.7 \mathrm{~Hz}, 1.4 \mathrm{~Hz}$, 1H), $7.23(\mathrm{~d}, J=7.6 \mathrm{~Hz}, 1 \mathrm{H}), 7.05(\mathrm{t}, J=8.7 \mathrm{~Hz}, 2 \mathrm{H}), 6.51(\mathrm{~s}, 1 \mathrm{H}), 1.99(\mathrm{~d}, J=1.5 \mathrm{~Hz}$, $3 \mathrm{H}), 1.79(\mathrm{~d}, J=1.5 \mathrm{~Hz}, 3 \mathrm{H}) \mathrm{ppm}$.

2-(2-Methylprop-1-en-1-yl)- $N$-(benzyloxy)benzamide (1s)

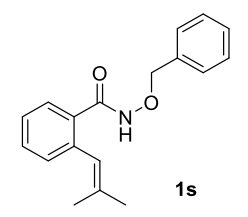

${ }^{1} \mathrm{H}$ NMR (CDCl, $\left.400 \mathrm{MHz}\right): \delta=8.68(\mathrm{~s}, 1 \mathrm{H}), 7.66(\mathrm{~d}, J=7.8 \mathrm{~Hz}, 1 \mathrm{H}), 7.43-7.34(\mathrm{~m}$, 6H), $7.24(\mathrm{td}, J=7.6 \mathrm{~Hz}, 1.5 \mathrm{~Hz}, 1 \mathrm{H}), 7.14(\mathrm{~d}, J=7.7 \mathrm{~Hz}, 1 \mathrm{H}), 6.27$ (s, 1H), 5.00 (s, 2H), $1.77(\mathrm{~d}, J=1.6 \mathrm{~Hz}, 3 \mathrm{H}), 1.63(\mathrm{~d}, J=1.4 \mathrm{~Hz}, 3 \mathrm{H}) \mathrm{ppm}$.

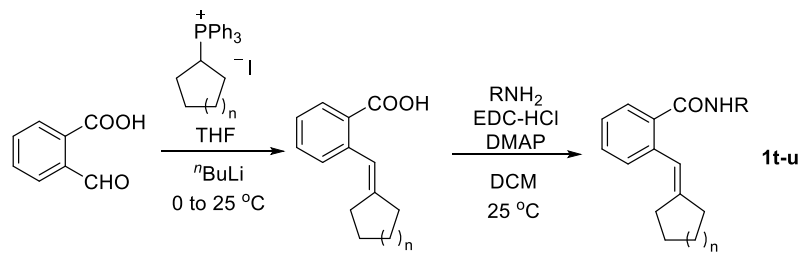

Typical Procedure: To a Schlenk tube charged with cyclohexyl or cyclopentyl triphenylphosphonium iodide $(0.65 \mathrm{mmol})$ was added anhydrous THF $(2 \mathrm{~mL})$ at $0{ }^{\circ} \mathrm{C}$ under argon. Then $n$-butyllithium $(0.6 \mathrm{mmol}$, 
$1 \mathrm{M}$ solution in cyclohexane) was added slowly. After stirring at $0{ }^{\circ} \mathrm{C}$ for 1 hour, a solution of 2-carboxybenzaldehyde $(0.5 \mathrm{mmol})$ in anhydrous THF $(0.5 \mathrm{~mL})$ was added, and the reaction mixture was stirred at $25{ }^{\circ} \mathrm{C}$ overnight. After quenched with saturated $\mathrm{NH}_{4} \mathrm{Cl}$ (aq.) and diluted with ethyl acetate, the aqueous layer was extracted with ethyl acetate twice. The combined organic phase was dried over $\mathrm{Na}_{2} \mathrm{SO}_{4}$, and concentrated in vacuo to give dark residue, which was then purified by flash chromatography using petroleum ether and ethyl acetate $(10: 1, v / v)$ as the eluent on silical gel to afford the 2-alkenylbenzoic acids shown in the above scheme in $25 \%$ yield from cyclohexyl triphenylphosphonium iodide and in $40 \%$ yield from cyclopentyl triphenylphosphonium iodide.

The second step was identical to the preparation of 10-s.

\section{2-(Cyclohexylidenemethyl)-N-phenylbenzamide (1t)}

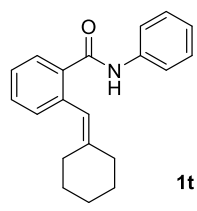

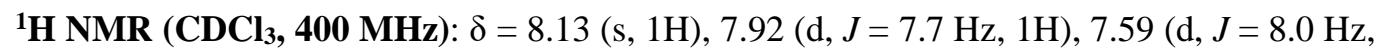
2H), 7.44-7.32 (m, 4H), 7.19 (d, $J=7.6 \mathrm{~Hz}, 1 \mathrm{H}), 7.13(\mathrm{t}, J=7.4 \mathrm{~Hz}, 1 \mathrm{H}), 6.45(\mathrm{~s}, 1 \mathrm{H}), 2.34$ 1t $(\mathrm{t}, J=6.0 \mathrm{~Hz}, 2 \mathrm{H}), 2.27(\mathrm{t}, J=5.9 \mathrm{~Hz}, 2 \mathrm{H}), 1.67-1.55(\mathrm{~m}, 6 \mathrm{H}) \mathrm{ppm}$.

\section{2-(Cyclopentylidenemethyl)-N-phenylbenzamide (1u)}

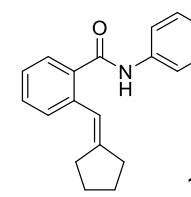

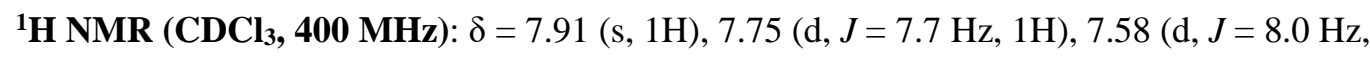
2H), 7.44-7.33 (m, 4H), 7.30-7.25 (m, 1H), $7.15(\mathrm{t}, J=7.4 \mathrm{~Hz}, 1 \mathrm{H}), 6.63(\mathrm{~s}, 1 \mathrm{H}), 2.51(\mathrm{t}, J$ 1u $=6.9 \mathrm{~Hz}, 2 \mathrm{H}), 2.42(\mathrm{t}, J=7.2 \mathrm{~Hz}, 2 \mathrm{H}), 1.75-1.69(\mathrm{~m}, 4 \mathrm{H}) \mathrm{ppm}$. 


\section{Detailed Results of Reaction Condition Screening}

Table S1. Detailed Results of Reaction Condition Screening ${ }^{[a]}$

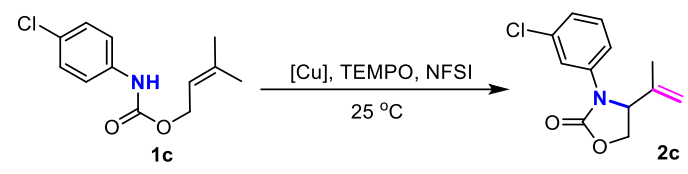

\begin{tabular}{lllllll}
\hline Entry & $\begin{array}{l}{[\mathbf{C u}]} \\
(\mathbf{m o l} \%)\end{array}$ & $\begin{array}{l}\text { TEMPO } \\
(\mathbf{m o l} \%)\end{array}$ & $\begin{array}{l}\text { NFSI } \\
(\text { equiv })\end{array}$ & $\begin{array}{l}\text { Solvent } \\
(\mathbf{2} \mathbf{~ m L})\end{array}$ & $\begin{array}{l}\text { Time } \\
(\mathbf{h})\end{array}$ & $\begin{array}{l}\text { Yield } \\
(\boldsymbol{\%})^{[\mathbf{b}]}\end{array}$ \\
\hline $\mathbf{1}$ & $\mathrm{CuCl}(10)$ & 20 & 2 & $\mathrm{MeCN}$ & 8 & 87 \\
$\mathbf{2}$ & - & 0 & 2 & $\mathrm{MeCN}$ & 8 & 0 \\
$\mathbf{3}$ & - & 20 & 0 & $\mathrm{MeCN}$ & 8 & 0 \\
$\mathbf{4}$ & $\mathrm{CuCl}(5)$ & 0 & 0 & $\mathrm{MeCN}$ & 8 & 0 \\
$\mathbf{5}$ & 0 & 20 & 2 & $\mathrm{MeCN}$ & 8 & 13 \\
$\mathbf{6}$ & $\mathrm{CuCl}(5)$ & 0 & 2 & $\mathrm{MeCN}$ & 8 & 40 \\
$\mathbf{7}$ & $\mathrm{CuCl}(5)$ & 20 & 0 & $\mathrm{MeCN}$ & 8 & 0 \\
$\mathbf{8}$ & $\mathrm{CuCl}(5)$ & 20 & 1.5 & $\mathrm{MeCN}$ & 8 & 78 \\
$\mathbf{9}$ & $\mathrm{CuCl}(5)$ & 20 & 1 & $\mathrm{MeCN}$ & 8 & 60 \\
$\mathbf{1 0}$ & $\mathrm{CuCl}(5)$ & 20 & 1.5 & $\mathrm{MeCN}$ & 12 & 89 \\
$\mathbf{1 1}$ & $\mathrm{CuCl}(5)$ & 20 & 1.5 & $\mathrm{MeCN}$ & 24 & 93 \\
$\mathbf{1 2}$ & $\mathrm{CuCl}(\mathbf{5})$ & $\mathbf{1 0}$ & $\mathbf{1 . 5}$ & $\mathrm{MeCN}$ & $\mathbf{1 2}$ & $\mathbf{8 9}$ \\
$\mathbf{1 3}$ & $\mathrm{CuCl}(5)$ & 10 & 1 & $\mathrm{MeCN}$ & 12 & 75 \\
$\mathbf{1 4}$ & - & 10 & 1.5 & $\mathrm{MeCN}$ & 12 & 11 \\
$\mathbf{1 5}$ & $\mathrm{CuCl}(5)$ & 0 & 1.5 & $\mathrm{MeCN}$ & 12 & 37 \\
$\mathbf{1 6}$ & $\mathrm{CuCl}(5)$ & 10 & 0 & $\mathrm{MeCN}$ & 12 & 0 \\
$\mathbf{1 0}$ & - & 0 & 1.5 & $\mathrm{MeCN}$ & 12 & 0 \\
\hline $\mathbf{1 9}$ & $\mathrm{CuCl}(5)$ & 10 & 1.5 & $\mathrm{EtOAc}$ & 12 & trace \\
\hline
\end{tabular}




\begin{tabular}{lllllll}
\hline $\mathbf{2 1}$ & $\mathrm{CuCl}(5)$ & 10 & 1.5 & $\mathrm{DMF}$ & 12 & 0 \\
$\mathbf{2 2}$ & $\mathrm{CuCl}(5)$ & 10 & 1.5 & $\mathrm{DCM}$ & 12 & trace \\
$\mathbf{2 3}$ & $\mathrm{CuCl}(5)$ & 10 & 1.5 & toluene & 12 & trace \\
$\mathbf{2 4}$ & $\mathrm{FeCl}_{2}(5)$ & 10 & 1.5 & $\mathrm{MeCN}$ & 12 & 15 \\
$\mathbf{2 5}$ & $\mathrm{CuI}(5)$ & 10 & 1.5 & $\mathrm{MeCN}$ & 12 & 64 \\
$\mathbf{2 6}$ & $\mathrm{CuCl}(5)$ & 10 & 1.5 & $\mathrm{MeCN}$ & 12 & 49 \\
$\mathbf{2 7}$ & $\mathrm{Cu}(\mathrm{OAc})_{2}(5)$ & 10 & 1.5 & $\mathrm{MeCN}$ & 12 & 53 \\
\hline
\end{tabular}

[a] Reaction conditions: carbamate $1 \mathbf{c}(0.3 \mathrm{mmol})$, NFSI, copper catalyst and TEMPO in solvent $(2 \mathrm{~mL})$ at $25{ }^{\circ} \mathrm{C}$ under argon $(1 \mathrm{~atm})$. See page S12 for the detailed experimental procedure. ${ }^{[\mathbf{b}]}$ Isolated yields of $\mathbf{2 c}$ on silica gel. 


\section{$\underline{\text { Experimental Procedure and Characterization Data }}$}

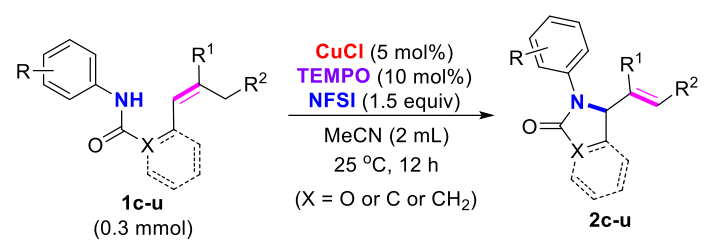

Typical Procedure: To a reaction tube charged with $\mathrm{CuCl}(1.5 \mathrm{mg}, 0.015 \mathrm{mmol})$ and NFSI (141.9 mg, 0.45 mmol) was added a solution of substrate $(\mathbf{1 c}-\mathbf{u}, 0.3 \mathrm{mmol})$ and TEMPO $(4.7 \mathrm{mg}, 0.03 \mathrm{mmol})$ in anhydrous $\mathrm{MeCN}(2 \mathrm{~mL})$ via a syringe under argon $(1 \mathrm{~atm})$. The reaction mixture was stirred at $25{ }^{\circ} \mathrm{C}$ for 12 hours (for 1c-n) or 30 minutes (for 1o-u). After quenched with $\mathrm{Na}_{2} \mathrm{CO}_{3}$ (aq.), the mixture was extracted with ethyl acetate. The combined organic phase was washed with water and brine, dried over $\mathrm{Na}_{2} \mathrm{SO}_{4}$, and concentrated in vacuo to give dark residue, which was then purified by flash chromatography using petroleum ether and ethyl acetate as the eluent on silical gel to afford formal aza-Wacker cyclization product 2c-u.

\section{3-(4-Chlorophenyl)-4-(prop-1-en-2-yl)oxazolidin-2-one (2c):}

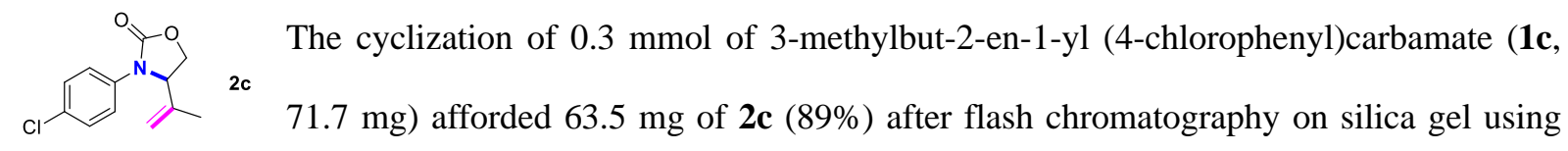
petroleum ether and ethyl acetate $(6: 1, v / v)$ as the eluent.

Colorless oil. ${ }^{1} \mathbf{H}$ NMR $\left(\mathbf{C D C l}_{3}, \mathbf{4 0 0} \mathbf{M H z}\right): \delta=7.46-7.42(\mathrm{~m}, 2 \mathrm{H}), 7.32-7.29(\mathrm{~m}, 2 \mathrm{H}), 5.09(\mathrm{~s}, 1 \mathrm{H}), 5.05$ (s, $1 \mathrm{H}), 4.85(\mathrm{dd}, J=9.0 \mathrm{~Hz}, 5.5 \mathrm{~Hz}, 1 \mathrm{H}), 4.56(\mathrm{t}, J=9.0 \mathrm{~Hz}, 1 \mathrm{H}), 4.11(\mathrm{dd}, J=8.8 \mathrm{~Hz}, 5.5 \mathrm{~Hz}, 1 \mathrm{H}), 1.67$ (s, 3H) ppm. ${ }^{13} \mathbf{C}$ NMR (CDCl 3,100 MHz): $\delta=155.41,140.94,135.86,129.86,128.96,121.35,116.51$, 66.12, 61.96, 16.37 ppm. HRMS $m / z$ (ESI) calcd for $\left[\mathrm{C}_{12} \mathrm{H}_{12} \mathrm{ClNO}_{2}+\mathrm{Na}\right]^{+} 260.0449$, found 260.0445 .

\section{3-(4-Bromophenyl)-4-(prop-1-en-2-yl)oxazolidin-2-one (2d):}

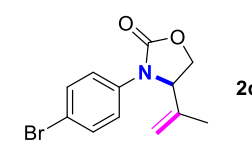

The cyclization of $0.3 \mathrm{mmol}$ of 3-methylbut-2-en-1-yl (4-bromophenyl)carbamate (1d, 2d

petroleum ether and ethyl acetate $(6: 1, v / v)$ as the eluent. 
Light yellow soild, m.p. $46.8-47.3{ }^{\circ} \mathrm{C} .{ }^{1} \mathbf{H}$ NMR $\left(\mathbf{C D C l}_{3}, \mathbf{4 0 0} \mathbf{M H z}\right): \delta=7.45(\mathrm{~d}, J=9.0 \mathrm{~Hz}, 2 \mathrm{H}), 7.39$ (d, $J=9.0 \mathrm{~Hz}, 2 \mathrm{H}), 5.09(\mathrm{~s}, 1 \mathrm{H}), 5.05(\mathrm{~s}, 1 \mathrm{H}), 4.86(\mathrm{dd}, J=9.0 \mathrm{~Hz}, 5.5 \mathrm{~Hz}, 1 \mathrm{H}), 4.57(\mathrm{t}, J=9.0 \mathrm{~Hz}, 1 \mathrm{H}), 4.12$ $(\mathrm{dd}, J=8.8 \mathrm{~Hz}, 5.5 \mathrm{~Hz}, 1 \mathrm{H}), 1.67$ (s, 3H) ppm. ${ }^{13} \mathbf{C} \mathbf{~ N M R}\left(\mathbf{C D C l}_{3}, \mathbf{1 0 0} \mathbf{M H z}\right): \delta=155.33,140.80,136.31$, $131.86,121.59,117.49,116.55,66.08,61.80,16.36 \mathrm{ppm}$. HRMS $\boldsymbol{m} / \boldsymbol{z}(\mathbf{E S I})$ calcd for $\left[\mathrm{C}_{12} \mathrm{H}_{12} \mathrm{BrNO}_{2}+\mathrm{Na}\right]^{+}$ 303.9944, found 303.9950.

\section{3-(4-Fluorophenyl)-4-(prop-1-en-2-yl)oxazolidin-2-one (2e):}

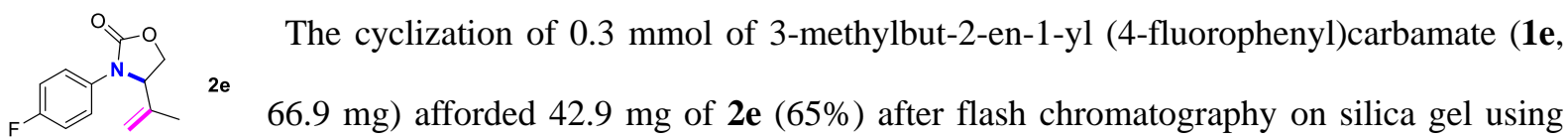
petroleum ether and ethyl acetate $(6: 1, v / v)$ as the eluent.

Light yellow oil. ${ }^{1} \mathbf{H}$ NMR $\left(\mathbf{C D C l}_{3}, \mathbf{4 0 0} \mathbf{M H z}\right): \delta=7.44(\mathrm{dd}, J=9.1 \mathrm{~Hz}, 4.7 \mathrm{~Hz}, 2 \mathrm{H}), 7.04(\mathrm{t}, J=8.7 \mathrm{~Hz}$, 2H), $5.09(\mathrm{~s}, 1 \mathrm{H}), 5.04(\mathrm{~s}, 1 \mathrm{H}), 4.86(\mathrm{dd}, J=9.1 \mathrm{~Hz}, 5.7 \mathrm{~Hz}, 1 \mathrm{H}), 4.57(\mathrm{t}, J=8.9 \mathrm{~Hz}, 1 \mathrm{H}), 4.13(\mathrm{dd}, J=8.8$ $\mathrm{Hz}, 5.7 \mathrm{~Hz}, 1 \mathrm{H}), 1.68(\mathrm{~s}, 3 \mathrm{H}) \mathrm{ppm} .{ }^{13} \mathbf{C} \mathbf{N M R}\left(\mathbf{C D C l}_{3}, \mathbf{1 0 0} \mathbf{M H z}\right): \delta=159.58(\mathrm{~J}=243.0 \mathrm{~Hz}), 155.75$, 140.92, $133.13(J=2.8 \mathrm{~Hz}), 122.25(J=32.0 \mathrm{~Hz}), 116.70,115.67(J=22.6 \mathrm{~Hz}), 66.00,62.32,16.28 \mathrm{ppm}$. HRMS $m / z$ (ESI) calcd for $\left[\mathrm{C}_{12} \mathrm{H}_{12} \mathrm{FNO}_{2}+\mathrm{Na}\right]^{+} 244.0744$, found 244.0749 .

\section{3-(4-(Trifluoromethyl)phenyl)-4-(prop-1-en-2-yl)oxazolidin-2-one (2f):}

OYo The cyclization of $0.3 \mathrm{mmol}$ of 3-methylbut-2-en-1-yl (4-(trifluoromethyl)phenyl) $2 f$ carbamate (1f, $81.9 \mathrm{mg})$ afforded $49.8 \mathrm{mg}$ of $\mathbf{2 f}(61 \%)$ after flash chromatography on silica gel using petroleum ether and ethyl acetate $(6: 1, v / v)$ as the eluent.

Light yellow soild, m.p. $46.6-47.1{ }^{\circ} \mathrm{C} .{ }^{1} \mathbf{H}$ NMR $\left(\mathbf{C D C l}_{3}, \mathbf{4 0 0} \mathbf{M H z}\right): \delta=7.65(\mathrm{~d}, J=8.9 \mathrm{~Hz}, 2 \mathrm{H}), 7.60(\mathrm{~d}$, $J=8.9 \mathrm{~Hz}, 2 \mathrm{H}), 5.13(\mathrm{~s}, 1 \mathrm{H}), 5.09(\mathrm{~s}, 1 \mathrm{H}), 4.91(\mathrm{dd}, J=9.1 \mathrm{~Hz}, 5.2 \mathrm{~Hz}, 1 \mathrm{H}), 4.60(\mathrm{t}, J=9.0 \mathrm{~Hz}, 1 \mathrm{H}), 4.15$ $(\mathrm{dd}, J=8.8 \mathrm{~Hz}, 5.2 \mathrm{~Hz}, 1 \mathrm{H}), 1.68(\mathrm{~s}, 3 \mathrm{H}) \mathrm{ppm} .{ }^{13} \mathbf{C} \mathbf{~ N M R}\left(\mathbf{C D C l}_{3}, \mathbf{1 0 0} \mathbf{M H z}\right): \delta=155.18,140.73,140.39$, $128.01,126.59,126.27,126.13,126.09,126.05,126.01,125.94,125.62,125.31,122.61,119.91,116.50$, 66.27, 61.64, $16.45 \mathrm{ppm}$. HRMS $\boldsymbol{m} / \boldsymbol{z}$ (ESI) calcd for $\left[\mathrm{C}_{13} \mathrm{H}_{12} \mathrm{~F}_{3} \mathrm{NO}_{2}+\mathrm{Na}\right]^{+} 294.0712$, found 294.0715.

3-(m-Tolyl)-4-(prop-1-en-2-yl)oxazolidin-2-one (2g):

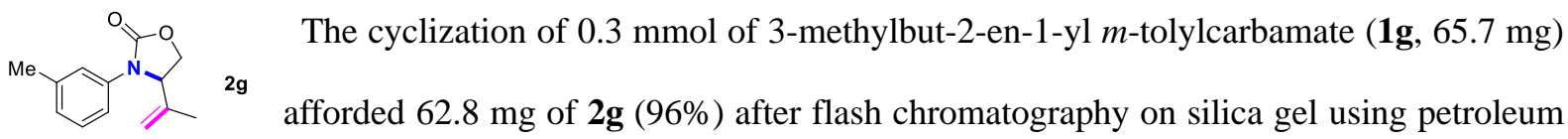
ether and ethyl acetate $(6: 1, v / v)$ as the eluent. 
Colorless oil. ${ }^{1} \mathbf{H}$ NMR $\left(\mathbf{C D C l}_{3}, \mathbf{4 0 0} \mathbf{M H z}\right): \delta=7.35(\mathrm{~s}, 1 \mathrm{H})$, 7.24-7.19 (m, 2H), 6.96-6.95 (m, 1H), 5.08 (s, 1H), $5.02(\mathrm{~s}, 1 \mathrm{H}), 4.88(d d, \mathrm{~J}=9.1 \mathrm{~Hz}, 5.5 \mathrm{~Hz}, 1 \mathrm{H}), 4.55(\mathrm{t}, J=9.0 \mathrm{~Hz}, 1 \mathrm{H}), 4.10(\mathrm{dd}, J=8.8 \mathrm{~Hz}, 5.5 \mathrm{~Hz}$, 1H), 2.35 (s, 3H), 1.68 (s, 3H) ppm. ${ }^{13} \mathbf{C}$ NMR ( $\left.\mathbf{C D C l}_{3}, \mathbf{1 0 0} \mathbf{~ M H z}\right): \delta=155.69,141.32,138.80,137.06$, 128.63, 125.54, 121.22, $117.40116 .13,66.09,62.07,21.55,16.44 \mathrm{ppm}$. HRMS $\mathbf{m} / \mathbf{z}$ (ESI) calcd for $\left[\mathrm{C}_{13} \mathrm{H}_{15} \mathrm{NO}_{2}+\mathrm{Na}\right]^{+} 240.0995$, found 240.1004 .

\section{3-(3,5-Dimethylphenyl)-4-(prop-1-en-2-yl)oxazolidin-2-one (2h):}

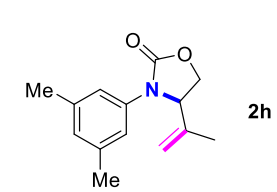

The cyclization of $0.3 \mathrm{mmol}$ of 3-methylbut-2-en-1-yl (3,5-dimethylphenyl)carbamate 2h $(\mathbf{1 h}, 69.9 \mathrm{mg})$ afforded $65.0 \mathrm{mg}$ of $\mathbf{2 h}(94 \%)$ after flash chromatography on silica gel using petroleum ether and ethyl acetate $(6: 1, v / v)$ as the eluent.

Light yellow oil. ${ }^{1} \mathbf{H}$ NMR (CDCl, 400 MHz): $\delta=7.08$ (s, 2H), 6.78 (s, 1H), 5.07 (s, 1H), 5.00 (s, 1H), $4.86(\mathrm{dd}, J=9.1 \mathrm{~Hz}, 5.5 \mathrm{~Hz}, 1 \mathrm{H}), 4.53(\mathrm{t}, J=8.9 \mathrm{~Hz}, 1 \mathrm{H}), 4.09$ (dd, $J=8.8 \mathrm{~Hz}, 5.5 \mathrm{~Hz}, 1 \mathrm{H}), 2.29(\mathrm{~s}, 6 \mathrm{H})$, $1.68(\mathrm{~s}, 3 \mathrm{H}) \mathrm{ppm} .{ }^{13} \mathbf{C} \mathbf{~ N M R}\left(\mathbf{C D C l}_{3}, \mathbf{1 0 0} \mathbf{M H z}\right): \delta=155.69,141.35,138.45,136.92,126.53,118.33$, 115.97, 66.04, 62.08, 21.39, $16.42 \mathrm{ppm}$. HRMS $\boldsymbol{m} / z$ (ESI) calcd for $\left[\mathrm{C}_{14} \mathrm{H}_{17} \mathrm{NO}_{2}+\mathrm{Na}\right]^{+} 254.1151$, found 254.1156 .

\section{3-(4-Isopropylphenyl)-4-(prop-1-en-2-yl)oxazolidin-2-one (2i):}

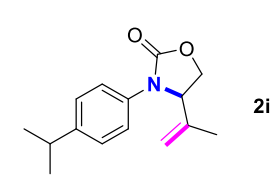

The cyclization of $0.3 \mathrm{mmol}$ of 3-methylbut-2-en-1-yl (4-isopropylphenyl)carbamate 2i (1i, $74.1 \mathrm{mg}$ ) afforded $60.8 \mathrm{mg}$ of $\mathbf{2 i}(83 \%)$ after flash chromatography on silica gel using petroleum ether and ethyl acetate $(6: 1, v / v)$ as the eluent.

Light yellow soild, m.p. 81.1-81.6 ${ }^{\circ} \mathrm{C} .{ }^{1} \mathbf{H}$ NMR $\left(\mathbf{C D C l}_{3}, \mathbf{4 0 0} \mathbf{M H z}\right): \delta=7.36(\mathrm{~d}, \mathrm{~J}=8.6 \mathrm{~Hz}, 2 \mathrm{H}), 7.17(\mathrm{~d}, \mathrm{~J}$ $=8.6 \mathrm{~Hz}, 2 \mathrm{H}), 5.06(\mathrm{~d}, J=0.9 \mathrm{~Hz}, 1 \mathrm{H}), 4.99(\mathrm{t}, J=1.4 \mathrm{~Hz}, 1 \mathrm{H}), 4.84(\mathrm{dd}, J=9.0 \mathrm{~Hz}, 5.6 \mathrm{~Hz}, 1 \mathrm{H}), 4.52(\mathrm{t}$, $J=8.9 \mathrm{~Hz}, 1 \mathrm{H}), 4.08(\mathrm{dd}, J=8.8 \mathrm{~Hz}, 5.6 \mathrm{~Hz}, 1 \mathrm{H}), 2.85$ (hept, $J=6.9 \mathrm{~Hz}, 1 \mathrm{H}), 1.67$ (s, 3H), $1.20(\mathrm{~d}, J=6.9$ $\mathrm{Hz}, 6 \mathrm{H}) \mathrm{ppm} .{ }^{13} \mathbf{C}$ NMR $\left(\mathbf{C D C l}_{\mathbf{3}}, \mathbf{1 0 0} \mathbf{M H z}\right): \delta=155.82,145.27,141.42,134.79,126.81,120.43,116.15$, 66.09, 62.15, 33.45, 23.87, $16.41 \mathrm{ppm}$. HRMS $\mathrm{m} / \mathrm{z}$ (ESI) calcd for $\left[\mathrm{C}_{15} \mathrm{H}_{19} \mathrm{NO}_{2}+\mathrm{Na}\right]^{+} 268.1308$, found 268.1308 .

\section{3-(2-Methoxyphenyl)-4-(prop-1-en-2-yl)oxazolidin-2-one (2j):}

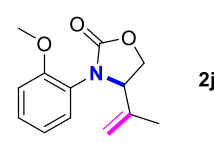

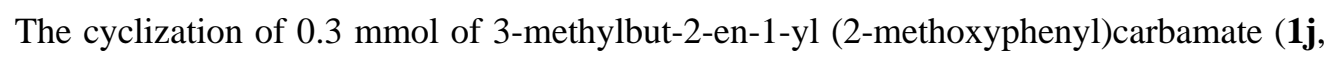


$70.5 \mathrm{mg}$ ) afforded $61.4 \mathrm{mg}$ of $\mathbf{2 j}$ ( $88 \%$ ) after flash chromatography on silica gel using petroleum ether and ethyl acetate $(6: 1, v / v)$ as the eluent.

Light yellow oil. ${ }^{1} \mathbf{H}$ NMR (DMSO-d $\left.6,400 \mathrm{MHz}\right): \delta=7.27(\mathrm{t}, J=8.2 \mathrm{~Hz}, 1 \mathrm{H}), 7.11(\mathrm{t}, J=2.2 \mathrm{~Hz}, 1 \mathrm{H}$ ), $7.06(\mathrm{dd}, J=8.1 \mathrm{~Hz}, 1.8 \mathrm{~Hz}, 1 \mathrm{H}), 6.72(\mathrm{dd}, J=8.2 \mathrm{~Hz}, 2.2 \mathrm{~Hz}, 1 \mathrm{H}), 5.13(\mathrm{dd}, J=9.0 \mathrm{~Hz}, 4.8 \mathrm{~Hz}, 1 \mathrm{H}), 5.06$ (s, 1H), $4.98(\mathrm{~s}, 1 \mathrm{H}), 4.57(\mathrm{t}, J=8.9 \mathrm{~Hz}, 1 \mathrm{H}), 4.14(\mathrm{dd}, J=8.7 \mathrm{~Hz}, 4.8 \mathrm{~Hz}, 1 \mathrm{H}), 3.73(\mathrm{~s}, 3 \mathrm{H}), 1.62(\mathrm{~s}, 3 \mathrm{H})$ ppm. ${ }^{13}$ C NMR (DMSO-d $\mathbf{6}, 100$ MHz): $\delta=159.47,154.98,141.52,138.49,129.58,115.46,112.45$, 109.31, 106.48, 65.72, 60.65, 55.10, $16.42 \mathrm{ppm}$. HRMS $\boldsymbol{m} / \boldsymbol{z}$ (ESI) calcd for $\left[\mathrm{C}_{13} \mathrm{H}_{15} \mathrm{NO}_{3}+\mathrm{Na}\right]^{+} 256.0944$, found 256.0944 .

\section{1-Phenyl-5-(prop-1-en-2-yl)pyrrolidin-2-one (2k):}

The cyclization of $0.3 \mathrm{mmol}$ of $N$-phenyl-5-methylhex-4-enamide (1k, $60.9 \mathrm{mg}$ ) afforded $\mathbf{2 k}$ $54.7 \mathrm{mg}$ of $\mathbf{2 k}(91 \%)$ after flash chromatography on silica gel using petroleum ether and ethyl acetate $(6: 1, v / v)$ as the eluent.

White soild, m.p. $105.3-105.8^{\circ} \mathrm{C} .{ }^{1} \mathbf{H}$ NMR $\left(\mathbf{C D C l}_{3}, \mathbf{4 0 0} \mathbf{M H z}\right): \delta=7.49-7.47(\mathrm{~m}, 2 \mathrm{H}), 7.37-7.33(\mathrm{~m}, 2 \mathrm{H})$, $7.16(\mathrm{t}, J=7.4 \mathrm{~Hz}, 1 \mathrm{H}), 4.94(\mathrm{~d}, J=0.6 \mathrm{~Hz}, 1 \mathrm{H}), 4.91-4.89(\mathrm{~m}, 1 \mathrm{H}), 4.69$ (dd, $J=8.2 \mathrm{~Hz}, 4.6 \mathrm{~Hz}, 1 \mathrm{H})$, 2.74-2.65 (m, 1H), 2.61-2.53 (m, 1H), 2.41-2.31 (m, 1H), 1.99-1.91 (m, 1H), $1.67(\mathrm{~s}, 3 \mathrm{H}) \mathrm{ppm} .{ }^{13} \mathbf{C}$ NMR $\left(\mathbf{C D C l}_{3}, \mathbf{1 0 0} \mathbf{M H z}\right): \delta=174.60,143.15,138.08,128.57,124.95,122.12,113.55,65.14,31.23,24.08,17.56$ ppm. HRMS $m / z$ (ESI) calcd for $\left[\mathrm{C}_{13} \mathrm{H}_{15} \mathrm{NO}+\mathrm{Na}\right]^{+} 224.1046$, found 224.1048 .

\section{(E)-1-Phenyl-5-(pent-2-en-3-yl)pyrrolidin-2-one (2l):}

The cyclization of $0.3 \mathrm{mmol}$ of $N$-phenyl-5-ethylhept-4-enamide (11, $69.3 \mathrm{mg}$ ) afforded 21 $40.4 \mathrm{mg}$ of $2 \mathbf{1}(59 \%)$ after flash chromatography on silica gel using petroleum ether and ethyl acetate $(6: 1, v / v)$ as the eluent.

Light yellow oil. ${ }^{1} \mathbf{H}$ NMR $\left(\mathbf{C D C l}_{\mathbf{3}}, \mathbf{4 0 0} \mathbf{M H z}\right): \delta=7.48(\mathrm{~d}, J=7.8 \mathrm{~Hz}, 2 \mathrm{H}), 7.32(\mathrm{t}, J=7.6 \mathrm{~Hz}, 2 \mathrm{H}), 7.11$ (t, $J=7.4 \mathrm{~Hz}, 1 \mathrm{H}), 5.36(\mathrm{q}, J=6.8 \mathrm{~Hz}, 1 \mathrm{H}), 4.62(\mathrm{dd}, J=8.2 \mathrm{~Hz}, 4.3 \mathrm{~Hz}, 1 \mathrm{H}), 2.69-2.60(\mathrm{~m}, 1 \mathrm{H})$, 2.53-2.45 (m, 1H), 2.35-2.25 (m, 1H), 2.17-2.08 (m, 1H), 1.98-1.86 (m, 2H), 1.57 (d, J=6.8 Hz, 3H), 0.96 (t, $J=7.6 \mathrm{~Hz}, 3 \mathrm{H})$ ppm. ${ }^{13} \mathbf{C} \mathbf{N M R}\left(\mathbf{C D C l}_{3}, \mathbf{1 0 0} \mathbf{M H z}\right): \delta=174.93,138.71,138.48,128.48,124.62,121.93$, 121.51, 65.14, 31.09, 25.03, 20.53, 13.06, $13.01 \mathrm{ppm}$. HRMS $\mathbf{m} / \mathbf{z}(\mathbf{E S I})$ calcd for $\left[\mathrm{C}_{15} \mathrm{H}_{19} \mathrm{NO}+\mathrm{Na}\right]^{+}$ 252.1359 , found 252.1362 . 
(E)-1-Phenyl-5-(hept-3-en-4-yl)pyrrolidin-2-one (2m):

The cyclization of $0.3 \mathrm{mmol}$ of $N$-phenyl-5-propyloct-4-enamide $(\mathbf{1 m}, 77.8 \mathrm{mg})$ 2m afforded $46.4 \mathrm{mg}$ of $\mathbf{2 m}(60 \%)$ after flash chromatography on silica gel using petroleum ether and ethyl acetate $(6: 1, v / v)$ as the eluent.

Light yellow oil. ${ }^{1} \mathbf{H}$ NMR $\left(\mathbf{C D C l}_{3}, \mathbf{4 0 0} \mathbf{M H z}\right): \delta=7.46(\mathrm{~d}, J=7.8 \mathrm{~Hz}, 2 \mathrm{H}), 7.31(\mathrm{t}, J=7.9 \mathrm{~Hz}, 2 \mathrm{H}), 7.11$ (t, $J=7.4 \mathrm{~Hz}, 1 \mathrm{H}), 5.27(\mathrm{t}, \mathrm{J}=7.3 \mathrm{~Hz}, 1 \mathrm{H}), 4.58(\mathrm{dd}, J=8.2 \mathrm{~Hz}, 4.4 \mathrm{~Hz}, 1 \mathrm{H}), 2.70-2.62(\mathrm{~m}, 1 \mathrm{H}), 2.54-2.46$ (m, 1H), 2.36-2.29 (m, 1H), 2.09-1.82 (m, 6H), 1.45-1.28 (m, 2H), $0.89(\mathrm{t}, J=7.3 \mathrm{~Hz}, 3 \mathrm{H}), 0.84(\mathrm{t}, J=7.5$ $\mathrm{Hz}, 3 \mathrm{H})$ ppm. ${ }^{13} \mathbf{C}$ NMR $\left(\mathbf{C D C l}_{3}, \mathbf{1 0 0} \mathbf{~ M H z}\right): \delta=175.02,138.45,135.68,129.72,128.44,124.72,122.18$, 65.18, 31.04, 30.30, 25.24, 22.36, 20.93, 14.48, $14.07 \mathrm{ppm}$. HRMS $\boldsymbol{m} / \boldsymbol{z}(\mathbf{E S I})$ calcd for $\left[\mathrm{C}_{17} \mathrm{H}_{23} \mathrm{NO}+\mathrm{Na}\right]^{+}$ 280.1672 , found 280.1671 .

\section{5-(Cyclohex-1-en-1-yl)-1-phenylpyrrolidin-2-one (2n):}

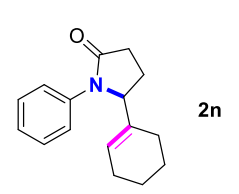

The cyclization of $0.3 \mathrm{mmol}$ of 4-cyclohexylidene- $N$-phenylbutanamide (1n, $72.9 \mathrm{mg}$ ) 2n afforded $58.3 \mathrm{mg}$ of $\mathbf{2 n}(81 \%)$ after flash chromatography on silica gel using petroleum ether and ethyl acetate $(6: 1, v / v)$ as the eluent.

White soild, m.p. $78.5-80.0{ }^{\circ} \mathrm{C} .{ }^{1} \mathbf{H}$ NMR $\left(\mathbf{C D C l}_{3}, \mathbf{4 0 0} \mathbf{M H z}\right): \delta=7.43(\mathrm{~d}, J=7.7 \mathrm{~Hz}, 2 \mathrm{H}), 7.34-7.30(\mathrm{~m}$, 2H), $7.13(\mathrm{t}, J=7.4 \mathrm{~Hz}, 1 \mathrm{H}), 5.62(\mathrm{~s}, 1 \mathrm{H}), 4.56(\mathrm{dd}, J=8.2 \mathrm{~Hz}, 3.4 \mathrm{~Hz}, 1 \mathrm{H}), 2.69-2.48(\mathrm{~m}, 2 \mathrm{H}), 2.33-2.24$ $(\mathrm{m}, 1 \mathrm{H}), 1.97-1.88(\mathrm{~m}, 4 \mathrm{H}), 1.78-1.75(\mathrm{~m}, 1 \mathrm{H}), 1.63-1.56(\mathrm{~m}, 1 \mathrm{H}), 1.51-1.42(\mathrm{~m}, 3 \mathrm{H}) \mathrm{ppm} .{ }^{13} \mathbf{C}$ NMR $\left(\mathbf{C D C l}_{3}, \mathbf{1 0 0} \mathrm{MHz}\right): \delta=174.69,138.20,135.78,128.46,125.24,124.85,122.46,65.90,31.46,24.83,24.29$, 23.49, 22.31, $22.23 \mathrm{ppm}$. HRMS $\boldsymbol{m} / \boldsymbol{z}$ (ESI) calcd for $\left[\mathrm{C}_{16} \mathrm{H}_{19} \mathrm{NO}+\mathrm{Na}\right]^{+} 264.1359$, found 264.1356.

\section{2-Phenyl-3-(prop-1-en-2-yl)isoindolin-1-one (20):}

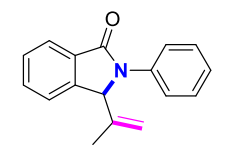

The cyclization of $0.3 \mathrm{mmol}$ of 2-(2-methylprop-1-en-1-yl)- $N$-phenylbenzamide (1o, $75.3 \mathrm{mg}$ ) afforded $67.4 \mathrm{mg}$ of $\mathbf{2 0}(90 \%)$ after flash chromatography on silica gel using petroleum ether and ethyl acetate $(10: 1, v / v)$ as the eluent.

White soild, m.p. 177.8-178.3 ${ }^{\circ} \mathrm{C} .{ }^{1} \mathbf{H}$ NMR $\left(\mathbf{C D C l}_{3}, \mathbf{4 0 0} \mathbf{M H z}\right): \delta=7.93(\mathrm{~d}, J=7.5 \mathrm{~Hz}, 1 \mathrm{H})$, 7.68-7.66 (m, 2H), $7.60(\mathrm{t}, J=7.5 \mathrm{~Hz}, 1 \mathrm{H}), 7.52(\mathrm{t}, J=7.3 \mathrm{~Hz}, 1 \mathrm{H}), 7.43-7.38(\mathrm{~m}, 3 \mathrm{H}), 7.19(\mathrm{t}, J=7.4 \mathrm{~Hz}, 1 \mathrm{H}), 5.62(\mathrm{~s}$, 1H), $5.38(\mathrm{~s}, 1 \mathrm{H}), 5.13(\mathrm{t}, J=1.4 \mathrm{~Hz}, 1 \mathrm{H}), 1.21(\mathrm{~s}, 3 \mathrm{H}) \mathrm{ppm} .{ }^{13} \mathbf{C} \mathbf{N M R}\left(\mathbf{C D C l}_{\mathbf{3}}, \mathbf{1 0 0} \mathbf{M H z}\right): \delta=167.59$, $143.20,141.78,137.63,132.29,132.22,128.82,128.78,125.01,123.93,122.41,122.34,117.28,67.83$, $15.44 \mathrm{ppm}$. HRMS $\boldsymbol{m} / \boldsymbol{z}$ (ESI) calcd for $\left[\mathrm{C}_{17} \mathrm{H}_{15} \mathrm{NO}+\mathrm{Na}\right]^{+} 272.1046$, found 272.1046. 


\section{2-(4-Methoxyphenyl)-3-(prop-1-en-2-yl)isoindolin-1-one (2p):}

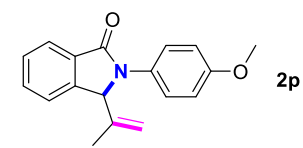

The cyclization of $0.3 \mathrm{mmol}$ of $N$-(4-methoxyphenyl)-2-(2-methylprop-1-en-1-yl) benzamide (1p, $84.3 \mathrm{mg}$ ) afforded $65.1 \mathrm{mg}$ of $\mathbf{2 p}(78 \%)$ after flash chromatography on silica gel using petroleum ether and ethyl acetate $(6: 1, v / v)$ as the eluent.

White soild, m.p. $121.4-121.9^{\circ} \mathrm{C} .{ }^{1} \mathbf{H}$ NMR $\left(\mathbf{C D C l}_{3}, \mathbf{4 0 0} \mathbf{M H z}\right): \delta=7.94(\mathrm{~d}, J=7.5 \mathrm{~Hz}, 1 \mathrm{H}), 7.62-7.51(\mathrm{~m}$, 4H), $7.42(\mathrm{dd}, J=7.5 \mathrm{~Hz}, 0.8 \mathrm{~Hz}, 1 \mathrm{H}), 7.96(\mathrm{~d}, J=9.1 \mathrm{~Hz}, 2 \mathrm{H}), 5.55$ (s, 1H), 5.36 (s, 1H), 5.13 (t, $J=1.4$ $\mathrm{Hz}, 1 \mathrm{H}), 3.83$ (s, 3H), 1.23 (s, 3H) ppm. ${ }^{13} \mathbf{C} \mathbf{~ N M R}\left(\mathbf{C D C l}_{3}, \mathbf{1 0 0} \mathbf{M H z}\right): \delta=167.45,156.93,143.13,141.65$, $132.27,132.05,130.45,128.68,124.18,123.76,122.34,117.41,114.06,68.22,55.33,15.38$ ppm. HRMS $\boldsymbol{m} / \boldsymbol{z}(\mathbf{E S I})$ calcd for $\left[\mathrm{C}_{18} \mathrm{H}_{17} \mathrm{NO}_{2}+\mathrm{Na}\right]^{+} 302.1151$, found 302.1149 .

\section{2-(4-(tert-Butyl)phenyl)-3-(prop-1-en-2-yl)isoindolin-1-one (2q):}

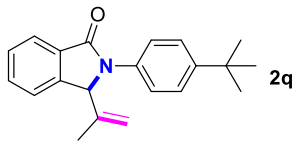

The cyclization of $0.3 \mathrm{mmol}$ of $N$-(4-(tert-butyl)phenyl)-2-(2-methylprop-1-en-1-yl) benzamide (1q, $92.2 \mathrm{mg}$ ) afforded $61.0 \mathrm{mg}$ of $\mathbf{2 q}(67 \%)$ after flash chromatography on silica gel using petroleum ether and ethyl acetate $(10: 1, v / v)$ as the eluent.

White soild, m.p. $185.9-186.4{ }^{\circ} \mathrm{C} .{ }^{1} \mathbf{H}$ NMR $\left(\mathbf{C D C l}_{3}, \mathbf{4 0 0} \mathbf{M H z}\right): \delta=7.93(\mathrm{~d}, J=7.5 \mathrm{~Hz}, 1 \mathrm{H}), 7.60-7.57(\mathrm{~m}$, 3H), $7.51(\mathrm{t}, J=7.4 \mathrm{~Hz}, 1 \mathrm{H}), 7.42-7.40(\mathrm{~m}, 3 \mathrm{H}), 5.59(\mathrm{~s}, 1 \mathrm{H}), 5.38(\mathrm{~s}, 1 \mathrm{H}), 5.13(\mathrm{~s}, 1 \mathrm{H}), 1.33(\mathrm{~s}, 9 \mathrm{H}), 1.23$ $(\mathrm{s}, 3 \mathrm{H}) \mathrm{ppm} .{ }^{13} \mathbf{C}$ NMR $\left(\mathbf{C D C l}_{3}, \mathbf{1 0 0} \mathbf{M H z}\right): \delta=167.56,147.74,143.21,141.94,134.93,132.29,132.14$, 128.69, 125.69, 123.83, 122.35, 121.82, 117.12, 67.81, 34.38, 31.29, $15.48 \mathrm{ppm}$. HRMS $\mathbf{m} / \mathbf{z}$ (ESI) calcd for $\left[\mathrm{C}_{21} \mathrm{H}_{23} \mathrm{NO}+\mathrm{H}\right]^{+}$306.1852, found 306.1858 .

\section{2-(4-Fluorophenyl)-3-(prop-1-en-2-yl)isoindolin-1-one (2r):}

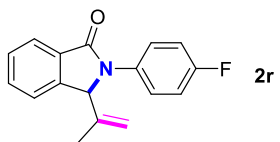

The cyclization of $0.3 \mathrm{mmol}$ of $N$-(4-fluorophenyl)-2-(2-methylprop-1-en-1-yl) benzamide (1r, $80.7 \mathrm{mg})$ afforded $51.9 \mathrm{mg}$ of $\mathbf{2 r}(65 \%)$ after flash chromatography on silica gel using petroleum ether and ethyl acetate $(6: 1, v / v)$ as the eluent.

White soild, m.p. $145.8-146.3{ }^{\circ} \mathrm{C} .{ }^{1} \mathbf{H}$ NMR ( $\left.\mathrm{CDCl}_{3}, 400 \mathrm{MHz}\right): \delta=7.95(\mathrm{~d}, J=7.5 \mathrm{~Hz}, 1 \mathrm{H}), 7.65-7.60(\mathrm{~m}$, 3H), $7.54(\mathrm{t}, J=7.3 \mathrm{~Hz}, 1 \mathrm{H}), 7.44(\mathrm{dd}, J=7.5 \mathrm{~Hz}, 0.8 \mathrm{~Hz}, 1 \mathrm{H}), 7.14-7.09(\mathrm{~m}, 2 \mathrm{H}), 5.57(\mathrm{~s}, 1 \mathrm{H}), 5.39(\mathrm{~s}$, 1H), $5.16(\mathrm{t}, J=1.4 \mathrm{~Hz}, 1 \mathrm{H}), 1.22(\mathrm{~s}, 3 \mathrm{H}) \mathrm{ppm} .{ }^{13} \mathbf{C} \mathbf{N M R}\left(\mathbf{C D C l}_{\mathbf{3}}, \mathbf{1 0 0} \mathbf{M H z}\right): \delta=167.54,159.85(\mathrm{~d}, J=$ $243.4 \mathrm{~Hz}), 142.23$ (d, $J=158.1 \mathrm{~Hz}), 133.56$ (d, $J=2.8 \mathrm{~Hz}), 132.38,131.95,128.84,124.05,124.02$ (d, $J=$ $22.0 \mathrm{~Hz}), 122.41,117.62,115.73,115.50,68.07,15.35 \mathrm{ppm}$. HRMS $\boldsymbol{m} / \boldsymbol{z}(\mathbf{E S I})$ calcd for $\left[\mathrm{C}_{17} \mathrm{H}_{14} \mathrm{FNO}+\mathrm{Na}\right]^{+}$ 


\section{2-Benzyloxy-3-(prop-1-en-2-yl)isoindolin-1-one (2s):}

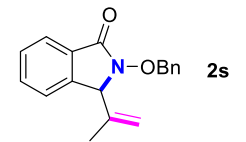

The cyclization of $0.3 \mathrm{mmol}$ of $N$-benzyloxy-2-(2-methylprop-1-en-1-yl)benzamide (1s, $84.3 \mathrm{mg}$ ) afforded $49.1 \mathrm{mg}$ of $2 \mathrm{~s}(59 \%)$ after flash chromatography on silica gel using petroleum ether and ethyl acetate $(6: 1, v / v)$ as the eluent.

Light yellow soild, m.p. 94.3-94.8 ${ }^{\circ} \mathrm{C} .{ }^{1} \mathbf{H}$ NMR $\left(\mathbf{C D C l}_{3}, 400 \mathbf{M H z}\right): \delta=7.86(\mathrm{~d}, J=7.4 \mathrm{~Hz}, 1 \mathrm{H})$, 7.55-7.45 (m, 4H), 7.37-7.36 (m, 3H), 7.21 (d, J=7.5 Hz, 1H), 5.19-5.09 (m, 4H), $4.73(\mathrm{~s}, 1 \mathrm{H}), 1.32(\mathrm{~s}, 3 \mathrm{H})$ ppm. ${ }^{13} \mathbf{C}$ NMR $\left(\mathbf{C D C l}_{3}, \mathbf{1 0 0} \mathbf{~ M H z}\right): \delta=165.50,141.08,140.60,135.26,132.22,129.95,129.53,128.80$, $128.61,128.42,123.59,122.42,118.02,78.06,67.92,15.61 \mathrm{ppm}$. HRMS $\mathbf{m} / \mathbf{z}$ (ESI) calcd for $\left[\mathrm{C}_{18} \mathrm{H}_{17} \mathrm{NO}+\mathrm{Na}\right]^{+} 302.1151$, found 302.1156 .

\section{3-(Cyclohex-1-en-1-yl)-2-phenylisoindolin-1-one (2t):}

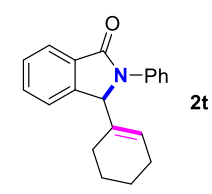

The cyclization of $0.3 \mathrm{mmol}$ of 2-(cyclohexylidenemethyl)- $N$-phenylbenzamide (1t, 87.3 $\mathrm{mg}$ ) afforded $64.8 \mathrm{mg}$ of $\mathbf{2 t}$ ( $75 \%$ ) after flash chromatography on silica gel using petroleum ether and ethyl acetate $(10: 1, v / v)$ as the eluent.

White soild, m.p. 166.3-166.9 ${ }^{\circ} \mathrm{C} .{ }^{1} \mathbf{H}$ NMR $\left(\mathbf{C D C l}_{3}, 400 \mathrm{MHz}\right): \delta=7.91(\mathrm{~d}, J=7.4 \mathrm{~Hz}, 1 \mathrm{H}), 7.64(\mathrm{~d}, J=$ $7.8 \mathrm{~Hz}, 2 \mathrm{H}), 7.58(\mathrm{t}, J=7.4 \mathrm{~Hz}, 1 \mathrm{H}), 7.49(\mathrm{t}, J=7.4 \mathrm{~Hz}, 1 \mathrm{H}), 7.41-7.37(\mathrm{~m}, 3 \mathrm{H}), 7.18(\mathrm{t}, J=7.4 \mathrm{~Hz}, 1 \mathrm{H})$, $6.07(\mathrm{~s}, 1 \mathrm{H}), 5.49(\mathrm{~s}, 1 \mathrm{H}), 2.07-2.05(\mathrm{~m}, 2 \mathrm{H}), 1.49-1.18(\mathrm{~m}, 6 \mathrm{H}) \mathrm{ppm} .{ }^{13} \mathbf{C} \mathbf{N M R}\left(\mathbf{C D C l}_{3}, \mathbf{1 0 0} \mathbf{M H z}\right): \delta=$ $167.66,143.96,137.69,134.25,132.20,132.11,129.21,128.65,128.46,124.83,123.71,122.53,122.35$, 68.36, 25.24, 22.05, 22.01, $21.59 \mathrm{ppm}$. HRMS $\mathbf{m} / z$ (ESI) calcd for $\left[\mathrm{C}_{20} \mathrm{H}_{19} \mathrm{NO}+\mathrm{Na}\right]^{+} 312.1359$, found 312.1356.

\section{3-(Cyclopent-1-en-1-yl)-2-phenylisoindolin-1-one (2u):}

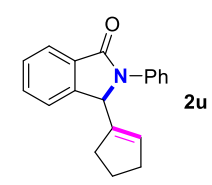

The cyclization of $0.3 \mathrm{mmol}$ of 2-(cyclopentylidenemethyl)- $N$-phenylbenzamide (1u, 83.1 $\mathrm{mg}$ ) afforded $35.4 \mathrm{mg}$ of $\mathbf{2 u}(43 \%)$ after flash chromatography on silica gel using petroleum ether and ethyl acetate $(10: 1, v / v)$ as the eluent.

White soild, m.p. $180.4-180.9^{\circ} \mathrm{C} .{ }^{1} \mathbf{H}$ NMR $\left(\mathbf{C D C l}_{3}, \mathbf{4 0 0} \mathbf{M H z}\right): \delta=7.93(\mathrm{~d}, J=7.4 \mathrm{~Hz}, 1 \mathrm{H}), 7.66(\mathrm{~d}, J=$ $8.4 \mathrm{~Hz}, 2 \mathrm{H}), 7.59(\mathrm{t}, J=7.4 \mathrm{~Hz}, 1 \mathrm{H}), 7.51(\mathrm{t}, J=7.4 \mathrm{~Hz}, 1 \mathrm{H}), 7.41-7.38(\mathrm{~m}, 3 \mathrm{H}), 7.18(\mathrm{t}, J=7.4 \mathrm{~Hz}, 1 \mathrm{H})$, $6.01(\mathrm{~s}, 1 \mathrm{H}), 5.86(\mathrm{~s}, 1 \mathrm{H}), 2.32-2.30(\mathrm{~m}, 2 \mathrm{H}), 1.88-1.66(\mathrm{~m}, 4 \mathrm{H}) \mathrm{ppm} .{ }^{13} \mathbf{C} \mathbf{N M R}\left(\mathbf{C D C l}_{3}, \mathbf{1 0 0} \mathbf{M H z}\right): \delta=$ 
$167.47,143.55,140.48,137.79,132.15,132.07,131.67,128.76,128.53,124.88,123.92,122.46,122.28$, 62.40, 32.34, 29.19, 22.85 ppm. HRMS $\boldsymbol{m} / \boldsymbol{z}$ (ESI) calcd for $\left[\mathrm{C}_{19} \mathrm{H}_{17} \mathrm{NO}+\mathrm{Na}\right]^{+} 298.1202$, found 298.1198 . 


\section{$\underline{\text { Kinetic Studies }}$}

\section{(1) Kinetic profiles for conversion from $1 \mathrm{i}$ to $2 \mathrm{i}$ under standard conditions}

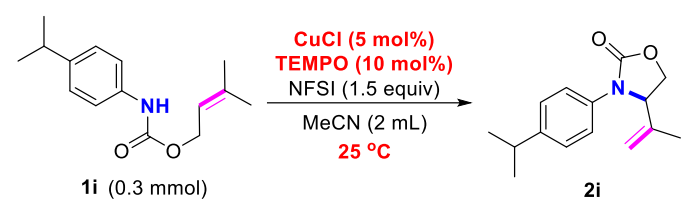

Typical Procedure: To a reaction tube charged with $\mathrm{CuCl}(1.5 \mathrm{mg}, 0.015 \mathrm{mmol})$ and NFSI $(141.9 \mathrm{mg}, 0.45$ mmol) was added a solution of substrate $1 \mathbf{i}(74.1 \mathrm{mg}, 0.3 \mathrm{mmol})$ and TEMPO $(4.7 \mathrm{mg}, 0.03 \mathrm{mmol})$ in anhydrous $\mathrm{MeCN}(2 \mathrm{~mL})$ via a syringe under argon $(1 \mathrm{~atm})$. The reaction mixture was stirred at $25{ }^{\circ} \mathrm{C}$ for designated time as shown in Table S2. After quenched with $\mathrm{Na}_{2} \mathrm{CO}_{3}$ (aq.), the mixture was extracted with ethyl acetate. The combined organic phase was concentrated in vacuo to give dark residue, which was then analyzed with ${ }^{1} \mathrm{H}-\mathrm{NMR}$ to determine the recoveries of $\mathbf{1} \mathbf{i}$ and the yield of $\mathbf{2} \mathbf{i}$ as summarized in Table $\mathrm{S} 2$, using 3,4,5-trichloropyridine as an internal standard.

Table S2. Detailed Results for Coversion from 1i to 2i under Standard Conditions

\begin{tabular}{|c|c|c|c|c|c|c|c|c|c|c|c|c|c|c|}
\hline $\begin{array}{l}\text { Time } \\
\text { (min) }\end{array}$ & 5 & 10 & 20 & 30 & 40 & 50 & 60 & 120 & 180 & 240 & 360 & 480 & 600 & 720 \\
\hline $\begin{array}{l}\text { Recovery } \\
\text { of } 1 \mathbf{i}\end{array}$ & 67.41 & 50.27 & 31.04 & 17.48 & 7.68 & 5.12 & 3.40 & 0 & 0 & 0 & 0 & 0 & $\mathbf{0}$ & 0 \\
\hline $\begin{array}{l}\text { Yield of } \\
2 \mathrm{i}\end{array}$ & 24.70 & 39.89 & 49.78 & 55.78 & 63.23 & 58.95 & 71.51 & 81.78 & 84.17 & 85.78 & 88.10 & 87.70 & 88.00 & 89.17 \\
\hline
\end{tabular}

(2) Kinetic profiles for conversion from $1 \mathrm{i}$ to $2 \mathrm{i}$ in the absence of TEMPO (with $\mathrm{CuCl}$ as the only catalyst)

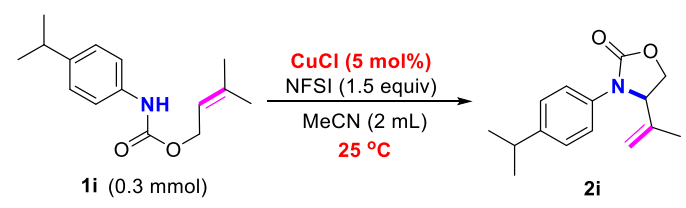

Typical Procedure: To a reaction tube charged with $\mathrm{CuCl}(1.5 \mathrm{mg}, 0.015 \mathrm{mmol})$ and NFSI (141.9 mg, 0.45 mmol) was added a solution of substrate $1 \mathbf{i}(74.1 \mathrm{mg}, 0.3 \mathrm{mmol})$ in anhydrous $\mathrm{MeCN}$ ( $2 \mathrm{~mL}$ ) via a syringe under argon $(1 \mathrm{~atm})$. The reaction mixture was stirred at $25{ }^{\circ} \mathrm{C}$ for designated time as shown in Table S3. After quenched with $\mathrm{Na}_{2} \mathrm{CO}_{3}$ (aq.), the mixture was extracted with ethyl acetate. The combined organic phase was concentrated in vacuo to give dark residue, which was then analyzed with ${ }^{1} \mathrm{H}-\mathrm{NMR}$ to determine the recoveries of $\mathbf{1 i}$ and the yield of $\mathbf{2} \mathbf{i}$ as summarized in Table S3, using 3,4,5-trichloropyridine as an 
internal standard.

Table S3. Detailed Results for Coversion from $1 \mathrm{i}$ to $2 \mathrm{i}$ with $\mathrm{CuCl}$ as the Only Catalyst

\begin{tabular}{lllllllllllllll}
\hline $\begin{array}{l}\text { Time } \\
(\min )\end{array}$ & 5 & 10 & 20 & 30 & 40 & 50 & 60 & 120 & 180 & 240 & 360 & 480 & 600 & 720 \\
\hline $\begin{array}{l}\text { Recovery } \\
\text { of 1i }\end{array}$ & 61.77 & 52.73 & 34.50 & 20.53 & 8.49 & 4.16 & 3.01 & 0 & 0 & 0 & 0 & 0 & 0 & 0 \\
$\begin{array}{l}\text { Yield of } \\
2 \mathrm{i}\end{array}$ & 7.23 & 13.08 & 21.89 & 26.40 & 27.43 & 27.02 & 26.88 & 27.81 & 29.58 & 27.27 & 29.02 & 28.77 & 28.56 & 29.00 \\
\hline
\end{tabular}

(3) Kinetic profiles for conversion from $1 \mathrm{i}$ to $2 \mathrm{i}$ in the absence of $\mathrm{CuCl}$ (with TEMPO as the only catalyst) as $25^{\circ} \mathrm{C}$

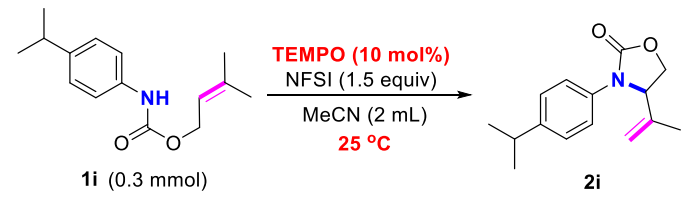

Typical Procedure: To a reaction tube charged with NFSI (141.9 mg, $0.45 \mathrm{mmol})$ was added a solution of substrate 1i $(74.1 \mathrm{mg}, 0.3 \mathrm{mmol})$ in anhydrous MeCN $(2 \mathrm{~mL})$ and TEMPO $(4.7 \mathrm{mg}, 0.03 \mathrm{mmol})$ via a syringe under argon $(1 \mathrm{~atm})$. The reaction mixture was stirred at $25{ }^{\circ} \mathrm{C}$ for designated time as shown in Table S4. After quenched with $\mathrm{Na}_{2} \mathrm{CO}_{3}$ (aq.), the mixture was extracted with ethyl acetate. The combined organic phase was concentrated in vacuo to give dark residue, which was then analyzed with ${ }^{1} \mathrm{H}-\mathrm{NMR}$ to determine the recoveries of $\mathbf{1 i}$ and the yield of $\mathbf{2 i}$ as summarized in Table S4, using 3,4,5-trichloropyridine as an internal standard.

Table S4. Detailed Results for Coversion from 1i to $2 \mathrm{i}$ with TEMPO as the Only Catalyst at $25^{\circ} \mathrm{C}$

\begin{tabular}{|c|c|c|c|c|c|c|c|c|c|c|c|c|c|c|}
\hline $\begin{array}{l}\text { Time } \\
(\mathrm{min})\end{array}$ & 5 & 10 & 20 & 30 & 40 & 50 & 60 & 120 & 180 & 240 & 360 & 480 & 600 & 720 \\
\hline $\begin{array}{l}\text { Recovery } \\
\text { of } 1 \mathbf{i}\end{array}$ & 95.99 & 94.05 & 90.45 & 87.46 & 85.21 & 83.38 & 81.84 & 79.33 & 78.50 & 77.29 & 75.13 & 75.91 & 75.34 & 74.10 \\
\hline $\begin{array}{l}\text { Yield of } \\
2 \mathrm{i}\end{array}$ & 1.98 & 2.67 & 4.73 & 7.81 & 9.09 & 9.64 & 9.70 & 10.13 & 9.05 & 8.98 & 10.26 & 10.67 & 11.75 & 12.60 \\
\hline
\end{tabular}

(4) Kinetic profiles for conversion from $1 \mathrm{i}$ to $2 \mathrm{i}$ in the absence of $\mathrm{CuCl}$ (with TEMPO as the only catalyst) as $50^{\circ} \mathrm{C}$

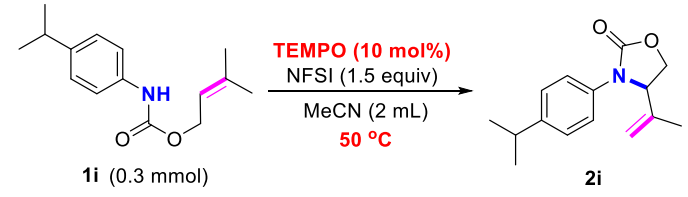

Typical Procedure: To a reaction tube charged with NFSI (141.9 mg, $0.45 \mathrm{mmol})$ was added a solution of 
substrate $1 \mathbf{i}(74.1 \mathrm{mg}, 0.3 \mathrm{mmol})$ in anhydrous $\mathrm{MeCN}(2 \mathrm{~mL})$ and TEMPO (4.7 $\mathrm{mg}, 0.03 \mathrm{mmol})$ via a syringe under argon $(1 \mathrm{~atm})$. The reaction mixture was stirred at $50{ }^{\circ} \mathrm{C}$ for designated time as shown in Table S5. After quenched with $\mathrm{Na}_{2} \mathrm{CO}_{3}$ (aq.), the mixture was extracted with ethyl acetate. The combined organic phase was concentrated in vacuo to give dark residue, which was then analyzed with ${ }^{1} \mathrm{H}-\mathrm{NMR}$ to determine the recoveries of $\mathbf{1 i}$ and the yield of $\mathbf{2 i}$ as summarized in Table S5, using 3,4,5-trichloropyridine as an internal standard.

Table S5. Detailed Results for Coversion from $1 \mathrm{i}$ to $2 \mathrm{i}$ with TEMPO as the Only Catalyst at $50{ }^{\circ} \mathrm{C}$

\begin{tabular}{|c|c|c|c|c|c|c|c|c|c|c|c|c|c|c|}
\hline $\begin{array}{l}\text { Time } \\
(\min )\end{array}$ & 5 & 10 & 20 & 30 & 40 & 50 & 60 & 120 & 180 & 240 & 360 & 480 & 600 & 720 \\
\hline $\begin{array}{l}\text { Recovery } \\
\text { of } 1 \mathbf{i}\end{array}$ & 90.13 & 83.99 & 78.49 & 75.09 & 73.26 & 70.88 & 69.30 & 67.33 & 65.46 & 60.61 & 52.78 & 48.69 & 50.62 & 46.06 \\
\hline $\begin{array}{l}\text { Yield of } \\
2 \mathrm{i}\end{array}$ & 3.22 & 7.08 & 11.76 & 13.29 & 15.08 & 16.80 & 16.71 & 16.76 & 15.27 & 15.55 & 15.29 & 15.61 & 16.20 & 16.43 \\
\hline
\end{tabular}

(5) Kinetic profiles for conversion from $1 \mathrm{i}$ to $2 \mathrm{i}$ in the absence of $\mathrm{CuCl}$ (with TEMPO as the only catalyst) as $80^{\circ} \mathrm{C}$

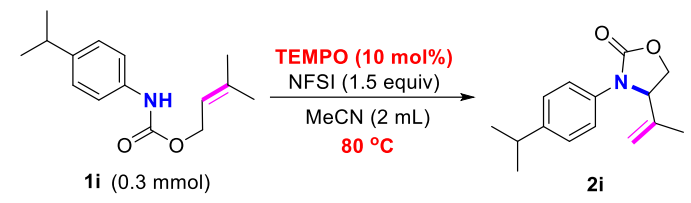

Typical Procedure: To a reaction tube charged with NFSI (141.9 mg, $0.45 \mathrm{mmol})$ was added a solution of substrate 1i $(74.1 \mathrm{mg}, 0.3 \mathrm{mmol})$ in anhydrous MeCN $(2 \mathrm{~mL})$ and TEMPO $(4.7 \mathrm{mg}, 0.03 \mathrm{mmol})$ via a syringe under argon $(1 \mathrm{~atm})$. The reaction mixture was stirred at $80{ }^{\circ} \mathrm{C}$ for designated time as shown in Table S6. After quenched with $\mathrm{Na}_{2} \mathrm{CO}_{3}$ (aq.), the mixture was extracted with ethyl acetate. The combined organic phase was concentrated in vacuo to give dark residue, which was then analyzed with ${ }^{1} \mathrm{H}-\mathrm{NMR}$ to determine the recoveries of $\mathbf{1 i}$ and the yield of $\mathbf{2} \mathbf{i}$ as summarized in Table S6, using 3,4,5-trichloropyridine as an internal standard.

Table S6. Detailed Results for Coversion from 1i to $2 \mathrm{i}$ with TEMPO as the Only Catalyst at $80^{\circ} \mathrm{C}$

\begin{tabular}{|c|c|c|c|c|c|c|c|c|c|c|c|c|c|c|}
\hline $\begin{array}{l}\text { Time } \\
(\min )\end{array}$ & 5 & 10 & 20 & 30 & 40 & 50 & 60 & 120 & 180 & 240 & 360 & 480 & 600 & 720 \\
\hline $\begin{array}{l}\text { Recovery } \\
\text { of } 1 \mathrm{i}\end{array}$ & 86.42 & 76.88 & 70.95 & 65.56 & 60.21 & 56.81 & 54.58 & 51.37 & 46.75 & 44.08 & 40.89 & 38.07 & 36.74 & 34.87 \\
\hline $\begin{array}{l}\text { Yield of } \\
2 \mathrm{i}\end{array}$ & 4.18 & 8.79 & 14.66 & 17.98 & 21.76 & 25.88 & 26.82 & 28.92 & 28.57 & 30.11 & 32.01 & 31.92 & 30.88 & 31.13 \\
\hline
\end{tabular}

(6) Kinetic profiles for conversion from 1 to $2 \mathrm{f}$ under standard conditions 


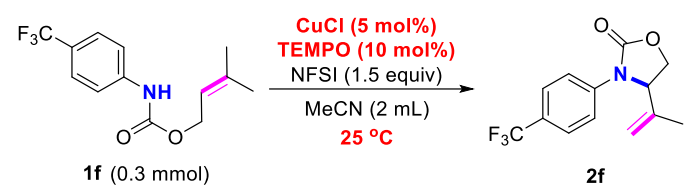

Typical Procedure: To a reaction tube charged with $\mathrm{CuCl}(1.5 \mathrm{mg}, 0.015 \mathrm{mmol})$ and NFSI (141.9 mg, 0.45 mmol) was added a solution of substrate $1 \mathbf{f}(81.9 \mathrm{mg}, 0.3 \mathrm{mmol})$ and TEMPO $(4.7 \mathrm{mg}, 0.03 \mathrm{mmol})$ in anhydrous MeCN $(2 \mathrm{~mL})$ via a syringe under argon $(1 \mathrm{~atm})$. The reaction mixture was stirred at $25{ }^{\circ} \mathrm{C}$ for designated time as shown in Table S7. After quenched with $\mathrm{Na}_{2} \mathrm{CO}_{3}$ (aq.), the mixture was extracted with ethyl acetate. The combined organic phase was concentrated in vacuo to give dark residue, which was then analyzed with ${ }^{1} \mathrm{H}-\mathrm{NMR}$ to determine the recoveries of $\mathbf{1 f}$ and the yield of $\mathbf{2} \mathbf{f}$ as summarized in Table S7, using 3,4,5-trichloropyridine as an internal standard.

Table S7. Detailed Results for Coversion from 1f to $2 \mathrm{f}$ under Standard Conditions

\begin{tabular}{|c|c|c|c|c|c|c|c|c|c|c|c|c|c|c|}
\hline $\begin{array}{l}\text { Time } \\
(\mathrm{min})\end{array}$ & 5 & 10 & 20 & 30 & 40 & 50 & 60 & 120 & 180 & 240 & 360 & 480 & 600 & 720 \\
\hline $\begin{array}{l}\text { Recovery } \\
\text { of } 1 f\end{array}$ & 75.01 & 62.67 & 55.21 & $\mathbf{5 0 . 7 7}$ & 45.59 & 40.10 & 36.41 & 24.96 & 20.54 & 16.04 & 12.92 & 7.35 & 3.04 & 0 \\
\hline $\begin{array}{l}\text { Yield of } \\
2 f\end{array}$ & 12.90 & 20.33 & 25.80 & 28.68 & 30.98 & 33.78 & 35.28 & 41.67 & 48.22 & 52.67 & $\mathbf{5 7 . 3 3}$ & 61.09 & 63.48 & 65.40 \\
\hline
\end{tabular}




\section{Control Experiments}

\section{(1) Reactions on carbamate 5 without alkenyl $\mathrm{C}=\mathrm{C}$ bond}

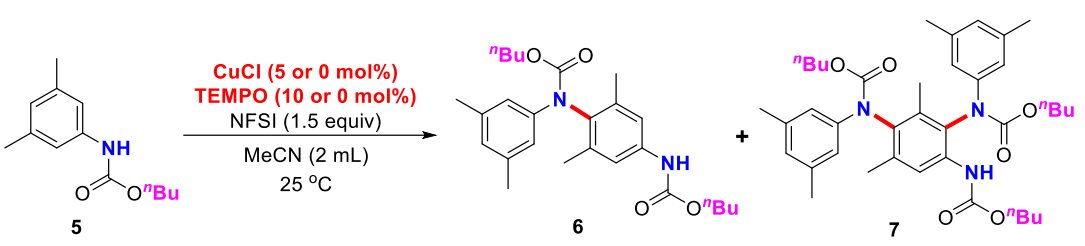

Typical Procedure (Scheme 4a, entry 1): To a reaction tube charged with $\mathrm{CuCl}(1.5 \mathrm{mg}, 0.015 \mathrm{mmol})$ and NFSI (141.9 mg, $0.45 \mathrm{mmol})$ was added a solution of carbamate $5(66.3 \mathrm{mg}, 0.3 \mathrm{mmol})$ and TEMPO (4.7 $\mathrm{mg}, 0.03 \mathrm{mmol})$ in anhydrous $\mathrm{MeCN}(2 \mathrm{~mL})$ via a syringe under argon $(1 \mathrm{~atm})$. The reaction mixture was stirred at $25{ }^{\circ} \mathrm{C}$ for 12 hours. After quenched with $\mathrm{Na}_{2} \mathrm{CO}_{3}$ (aq.), the mixture was extracted with ethyl acetate. The combined organic phase was washed with water and brine, dried over $\mathrm{Na}_{2} \mathrm{SO}_{4}$, and concentrated in vacuo to give dark residue, which was then purified by flash chromatography using petroleum ether and ethyl acetate as the eluent $(10: 1$ to $6: 1, v / v)$ on silical gel, affording $38.5 \mathrm{mg}$ of the C-H amination product 6 (58\%). The yield was calculated according to the maximum theoretical production of $\mathbf{6}$, which is $0.15 \mathrm{mmol}$.

Typical Procedure (Scheme 4a, entry 2): To a reaction tube charged with $\mathrm{CuCl}(1.5 \mathrm{mg}, 0.015 \mathrm{mmol})$ and NFSI $(141.9 \mathrm{mg}, 0.45 \mathrm{mmol})$ was added a solution of carbamate $5(66.3 \mathrm{mg}, 0.3 \mathrm{mmol})$ in anhydrous $\mathrm{MeCN}(2 \mathrm{~mL})$ via a syringe under argon $(1 \mathrm{~atm})$. The reaction mixture was stirred at $25{ }^{\circ} \mathrm{C}$ for 12 hours. After quenched with $\mathrm{Na}_{2} \mathrm{CO}_{3}$ (aq.), the mixture was extracted with ethyl acetate. The combined organic phase was washed with water and brine, dried over $\mathrm{Na}_{2} \mathrm{SO}_{4}$, and concentrated in vacuo to give dark residue, which was then purified by flash chromatography using petroleum ether and ethyl acetate as the eluent (10:1 to $6: 1, v / v)$ on silical gel, affording $9.4 \mathrm{mg}$ of the $\mathrm{C}-\mathrm{H}$ amination product $6(14 \%)$ and $28.3 \mathrm{mg}$ of the over-aminated product $7(42 \%)$. The yields were calculated according to the maximum theoretical productions of 6 and 7 , which are $0.15 \mathrm{mmol}$ and $0.1 \mathrm{mmol}$, respectively.

Typical Procedure (Scheme 4a, entry 3): To a reaction tube charged with NFSI (141.9 mg, $0.45 \mathrm{mmol}$ ) was added a solution of carbamate $5(66.3 \mathrm{mg}, 0.3 \mathrm{mmol})$ and TEMPO $(4.7 \mathrm{mg}, 0.03 \mathrm{mmol})$ in anhydrous $\operatorname{MeCN}(2 \mathrm{~mL})$ via a syringe under argon $(1 \mathrm{~atm})$. The reaction mixture was stirred at $25^{\circ} \mathrm{C}$ for 12 hours. No over-aminated product 7 was generated, while only trace amount of $\mathbf{6}$ could be observed on TLC. 
Butyl (4-((butoxycarbonyl)amino)-2,6-dimethylphenyl)(3,5-dimethylphenyl)carbamate (6):

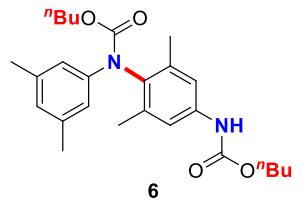

${ }^{1} \mathrm{H}$ NMR $\left(\mathbf{C D C l}_{3}, \mathbf{4 0 0} \mathbf{M H z}\right): \delta=7.16-7.14(\mathrm{~m}, 2 \mathrm{H}), 6.83(\mathrm{~s}, 2 \mathrm{H}), 6.70-6.68(\mathrm{~m}, 2 \mathrm{H})$, $4.17(\mathrm{t}, J=6.7 \mathrm{~Hz}, 2 \mathrm{H}), 4.13(\mathrm{t}, J=6.7 \mathrm{~Hz}, 2 \mathrm{H}), 2.23(\mathrm{~s}, 6 \mathrm{H}), 2.11(\mathrm{~s}, 6 \mathrm{H}), 1.66(\mathrm{p}, J$ $=7.1 \mathrm{~Hz}, 2 \mathrm{H}), 1.54(\mathrm{p}, J=7.1 \mathrm{~Hz}, 2 \mathrm{H}), 1.68-1.37(\mathrm{~m}, 2 \mathrm{H}), 1.29-1.22(\mathrm{~m}, 2 \mathrm{H}), 0.96(\mathrm{t}$, $J=7.4 \mathrm{~Hz}, 3 \mathrm{H}), 0.86(\mathrm{t}, J=7.4 \mathrm{~Hz}, 3 \mathrm{H}) \mathrm{ppm} .{ }^{13} \mathbf{C} \mathbf{N M R}\left(\mathbf{C D C l}_{3}, \mathbf{1 0 0} \mathbf{M H z}\right): \delta=154.65,153.69,141.00$, $137.91,137.24,136.99,134.65,125.87,120.29,118.21,65.70,65.07,30.93,30.86,21.40,19.02,18.94$, 18.22, 13.68, $13.59 \mathrm{ppm}$. HRMS $\boldsymbol{m} / z$ (ESI) calcd for $\left[\mathrm{C}_{26} \mathrm{H}_{36} \mathrm{~N}_{2} \mathrm{O}_{4}+\mathrm{H}\right]^{+} 441.2748$, found 441.2740 .

Dibutyl (4-((butoxycarbonyl)amino)-2,6-dimethyl-1,3-phenylene)bis((3,5-dimethylphenyl)carbamate) (7):

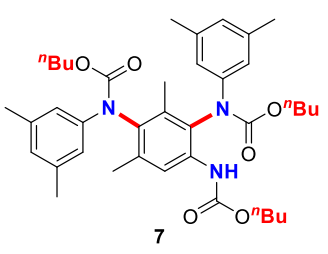

${ }^{1} \mathrm{H}$ NMR $\left(\mathrm{CDCl}_{3}, 400 \mathrm{MHz}\right): \delta=7.17(\mathrm{~s}, 2 \mathrm{H}), 6.96(\mathrm{~s}, 2 \mathrm{H}), 6.83(\mathrm{~s}, 2 \mathrm{H})$, 6.70-6.69 (m, 1H), $6.63(\mathrm{~s}, 1 \mathrm{H}), 4.18(\mathrm{t}, J=6.7 \mathrm{~Hz}, 2 \mathrm{H}), 4.14(\mathrm{t}, J=6.7 \mathrm{~Hz}, 2 \mathrm{H})$, $4.10(\mathrm{t}, J=6.5 \mathrm{~Hz}, 2 \mathrm{H}), 2.23(\mathrm{~s}, 6 \mathrm{H}), 2.12(\mathrm{~s}, 6 \mathrm{H}), 2.05(\mathrm{~s}, 6 \mathrm{H}), 1.70-1.63(\mathrm{~m}, 2 \mathrm{H})$, $1.56-1.39(\mathrm{~m}, 6 \mathrm{H}), 1.28-1.18(\mathrm{~m}, 4 \mathrm{H}), 0.96(\mathrm{t}, J=7.4 \mathrm{~Hz}, 3 \mathrm{H}), 0.86(\mathrm{t}, J=7.5 \mathrm{~Hz}$, 3H), $0.82(\mathrm{t}, J=7.3 \mathrm{~Hz}, 3 \mathrm{H}) \mathrm{ppm} .{ }^{13} \mathbf{C}$ NMR $\left(\mathbf{C D C l}_{3}, \mathbf{1 0 0} \mathbf{M H z}\right): \delta=154.59,153.69,140.88,140.14$, $137.94,137.27,137.13,136.58,135.30,125.88,122.06,120.26,118.27,65.85,65.62,65.15,30.94,30.90$, 30.81, 21.44, 19.04, 18.97, 18.93, 18.35, 18.22, 13.70, $13.60 \mathrm{ppm}$. HRMS $\mathbf{m} / \mathbf{z}$ (ESI) calcd for $\left[\mathrm{C}_{39} \mathrm{H}_{53} \mathrm{~N}_{3} \mathrm{O}_{6}+\mathrm{Na}\right]^{+}$682.3827, found 682.3833 .

\section{(2) Control experiments with $\mathrm{BrCCl}_{3}$}

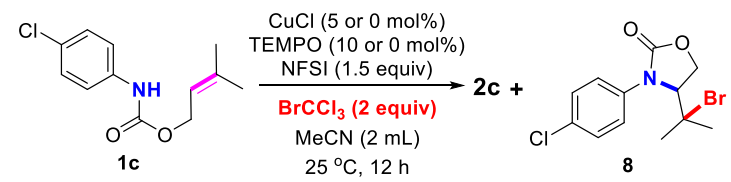

Typical Procedure (Scheme 4b, entry 1): To a reaction tube charged with $\mathrm{CuCl}(1.5 \mathrm{mg}, 0.015 \mathrm{mmol})$ and NFSI (141.9 mg, $0.45 \mathrm{mmol})$ was added a solution of carbamate 5 (66.3 mg, $0.3 \mathrm{mmol})$, TEMPO (4.7 mg, $0.03 \mathrm{mmol})$ and $\mathrm{BrCCl}_{3}(119.0 \mathrm{mg}, 60 \mu \mathrm{L}, 0.6 \mathrm{mmol})$ in anhydrous $\mathrm{MeCN}(2 \mathrm{~mL})$ via a syringe under $\operatorname{argon}(1 \mathrm{~atm})$. The reaction mixture was stirred at $25{ }^{\circ} \mathrm{C}$ for 12 hours. After quenched with $\mathrm{Na}_{2} \mathrm{CO}_{3}$ (aq.), the mixture was extracted with ethyl acetate. The combined organic phase was washed with water and brine, 
dried over $\mathrm{Na}_{2} \mathrm{SO}_{4}$, and concentrated in vacuo to give dark residue, which was then purified by flash chromatography using petroleum ether and ethyl acetate as the eluent $(10: 1$ to $6: 1, v / v)$ on silical gel, affording $37.4 \mathrm{mg}$ of the formal aza-Wacker cyclization product $\mathbf{2 c}(53 \%)$ and $10.7 \mathrm{mg}$ of the brominated product $8(11 \%)$.

Typical Procedure (Scheme 4b, entry 2): To a reaction tube charged with $\mathrm{CuCl}(1.5 \mathrm{mg}, 0.015 \mathrm{mmol})$ and NFSI (141.9 mg, $0.45 \mathrm{mmol})$ was added a solution of carbamate $5(66.3 \mathrm{mg}, 0.3 \mathrm{mmol})$ and $\mathrm{BrCCl}_{3}(119.0$ $\mathrm{mg}, 60 \mu \mathrm{L}, 0.6 \mathrm{mmol})$ in anhydrous $\mathrm{MeCN}(2 \mathrm{~mL})$ via a syringe under argon $(1 \mathrm{~atm})$. The reaction mixture was stirred at $25{ }^{\circ} \mathrm{C}$ for 12 hours. After quenched with $\mathrm{Na}_{2} \mathrm{CO}_{3}$ (aq.), the mixture was extracted with ethyl acetate. The combined organic phase was washed with water and brine, dried over $\mathrm{Na}_{2} \mathrm{SO}_{4}$, and concentrated in vacuo to give dark residue, which was then purified by flash chromatography using petroleum ether and ethyl acetate as the eluent $(10: 1$ to $6: 1, v / v)$ on silical gel, affording $60.5 \mathrm{mg}$ of the brominated product $\mathbf{8}(64 \%)$. Only trace amount of $\mathbf{2 c}$ could be observed on TLC.

Typical Procedure (Scheme 4b, entry 3): To a reaction tube charged with NFSI (141.9 mg, $0.45 \mathrm{mmol}$ ) was added a solution of carbamate $5(66.3 \mathrm{mg}, 0.3 \mathrm{mmol})$, TEMPO $(4.7 \mathrm{mg}, 0.03 \mathrm{mmol})$ and $\mathrm{BrCCl}_{3}(119.0$ $\mathrm{mg}, 60 \mu \mathrm{L}, 0.6 \mathrm{mmol})$ in anhydrous $\mathrm{MeCN}(2 \mathrm{~mL})$ via a syringe under argon $(1 \mathrm{~atm})$. The reaction mixture was stirred at $25^{\circ} \mathrm{C}$ for 12 hours, and no $2 \mathbf{c}$ or $\mathbf{8}$ could be observed on TLC.

\section{Rac-4-(2-Bromopropan-2-yl)-3-(4-chlorophenyl)oxazolidin-2-one (8):}

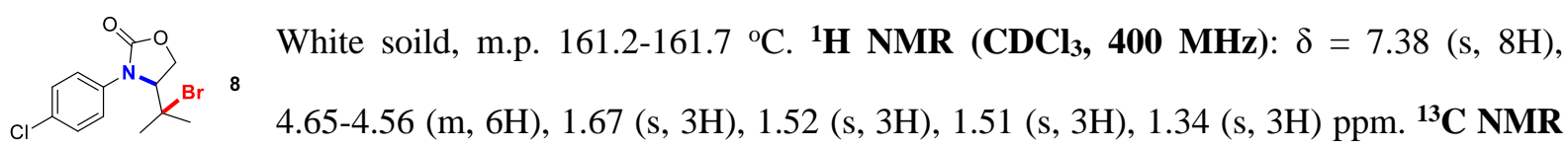
$\left(\mathbf{C D C l}_{3}, \mathbf{1 0 0} \mathbf{M H z}\right): \delta=156.23,156.15,136.29,136.23,132.07,131.99,129.50,129.47,125.41,125.38$, 70.31, 66.38, 66.15, 65.25, 65.01, 64.83, 32.82, 31.10, 27.33, $26.17 \mathrm{ppm}$. HRMS $\boldsymbol{m} / \boldsymbol{z}$ (ESI) calcd for $\left[\mathrm{C}_{12} \mathrm{H}_{13} \mathrm{BrClNO}{ }_{2}+\mathrm{Na}\right]^{+}$339.9710, found 339.9717. 


\section{Gram-scale Synthesis}

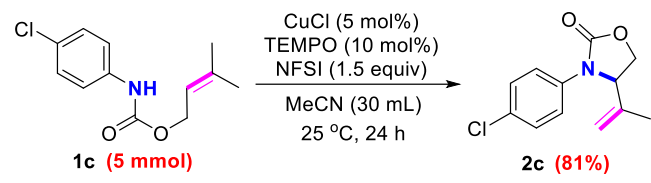

Typical Procedure (Scheme 5): To a three-necked flask charged with $\mathrm{CuCl}$ (24.8 mg, $0.25 \mathrm{mmol})$ and NFSI (2.365 g, $7.5 \mathrm{mmol})$ was added a solution of substrate 1c $(1.195 \mathrm{~g}, 5 \mathrm{mmol})$ and TEMPO (78.1 mg, $0.5 \mathrm{mmol})$ in anhydrous $\mathrm{MeCN}(30 \mathrm{~mL})$ via a syringe under argon $(1 \mathrm{~atm})$. The reaction mixture was stirred at $25{ }^{\circ} \mathrm{C}$ for 12 hours. After quenched with $\mathrm{Na}_{2} \mathrm{CO}_{3}$ (aq.), the mixture was extracted with ethyl acetate. The combined organic phase was washed with water and brine, dried over $\mathrm{Na}_{2} \mathrm{SO}_{4}$, and concentrated in vacuo to give dark residue, which was then purified by flash chromatography using petroleum ether and ethyl acetate as the eluent (10:1 to 6:1, v/v) on silical gel, affording $963.4 \mathrm{mg}$ of the formal aza-Wacker cyclization product $\mathbf{2 c}(81 \%)$ 


\section{References}

[1] Yi, X.; Hu, X. Angew. Chem. Int. Ed. 2019, 58, 4700-4704. 

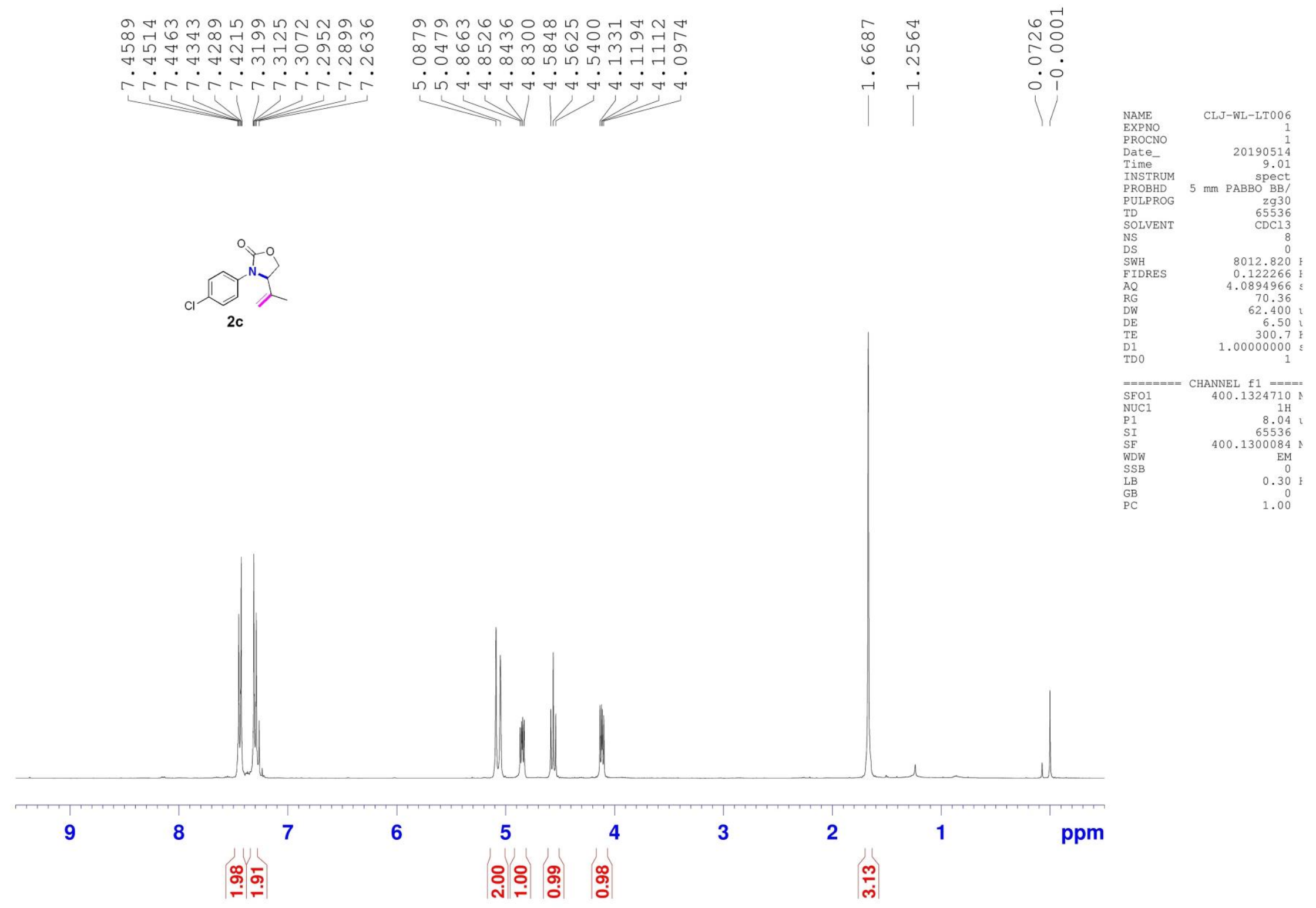

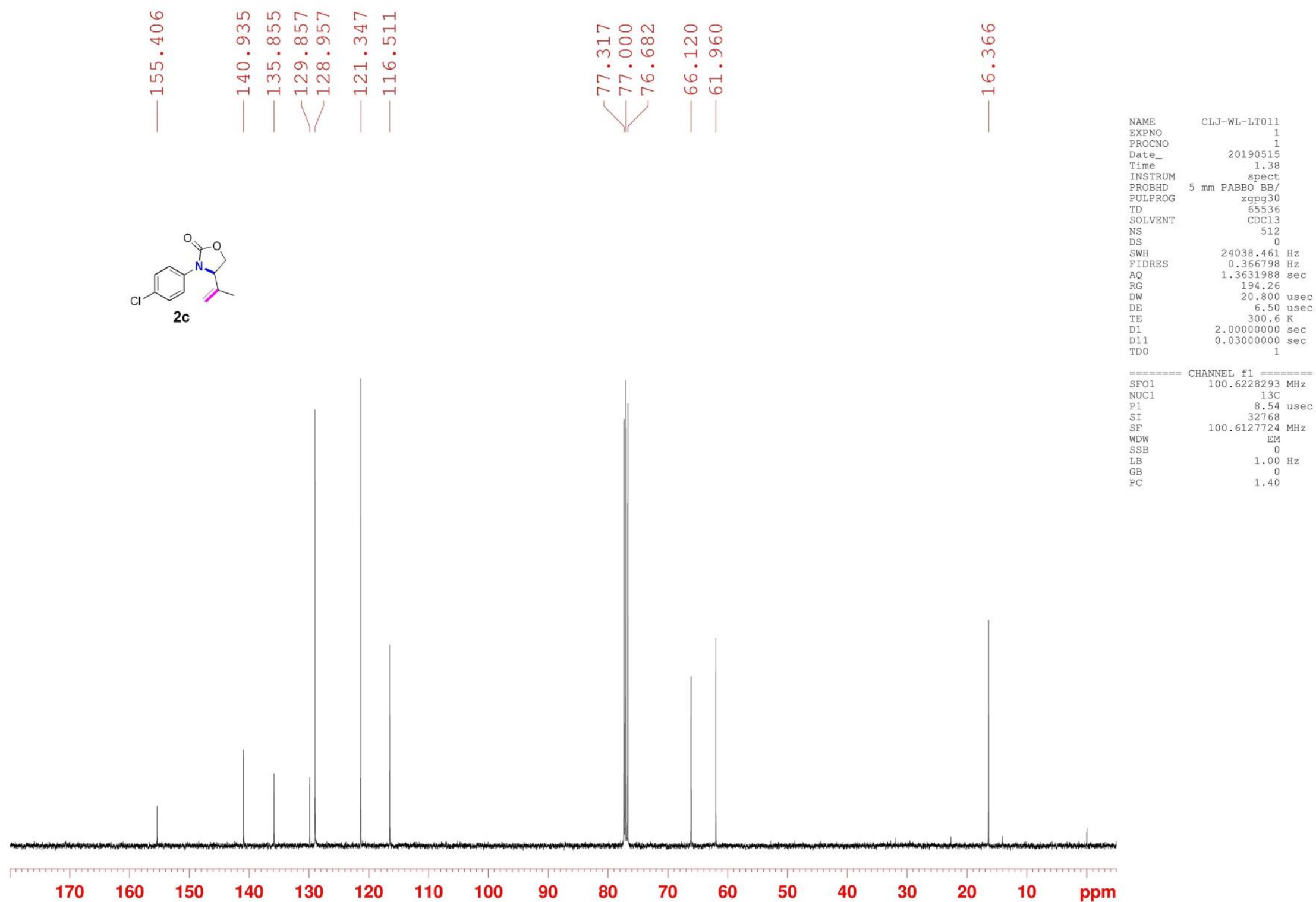


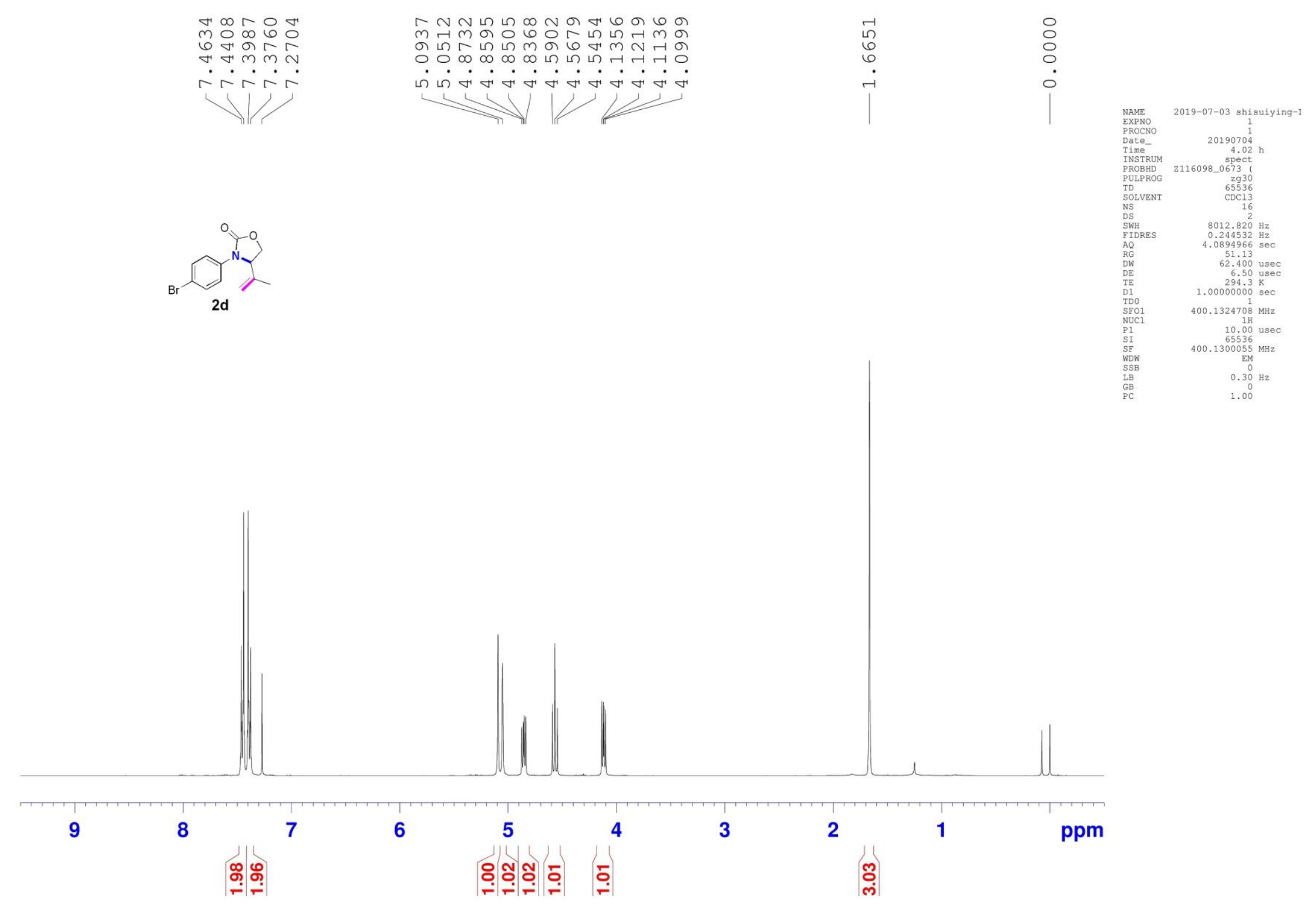



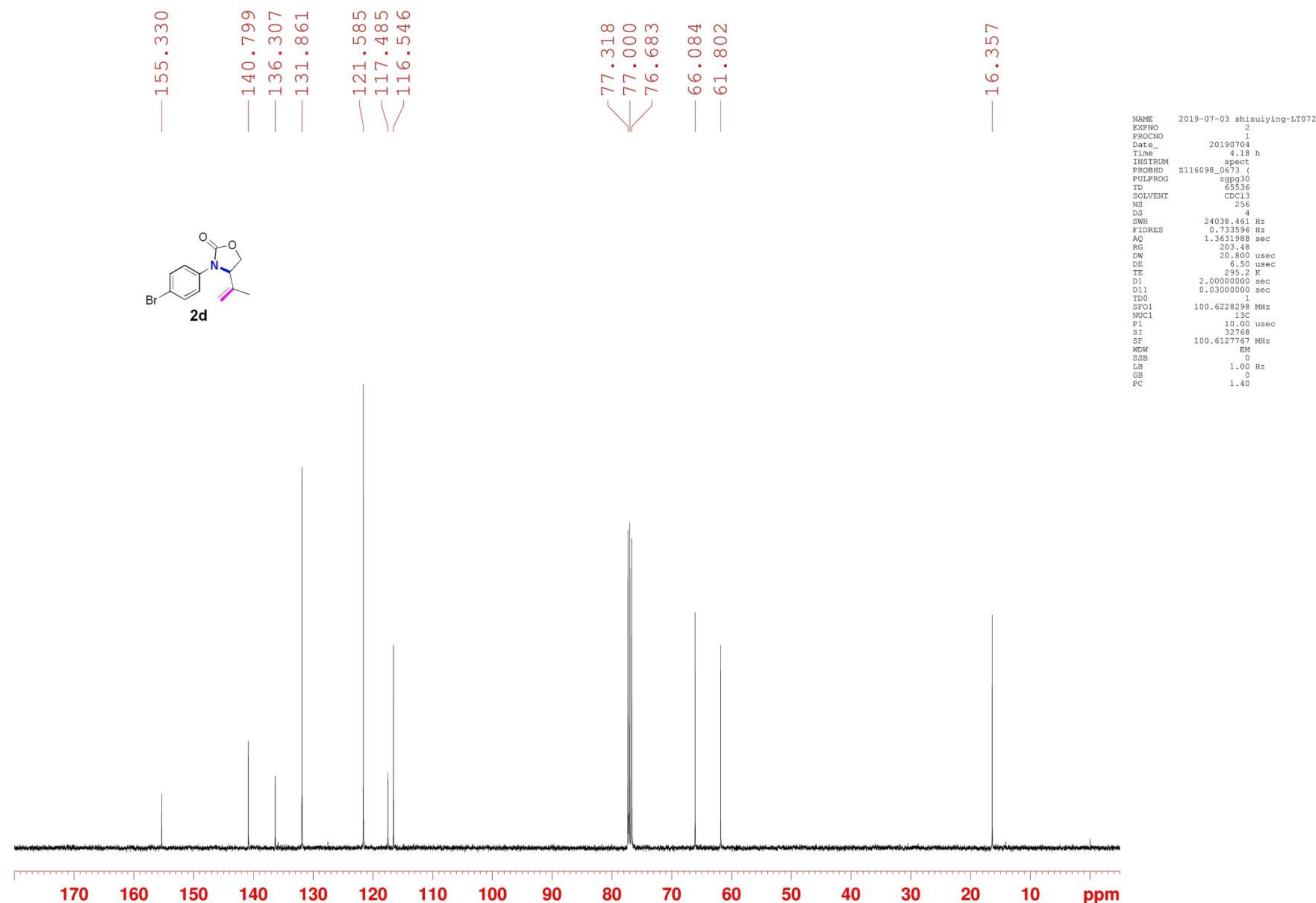


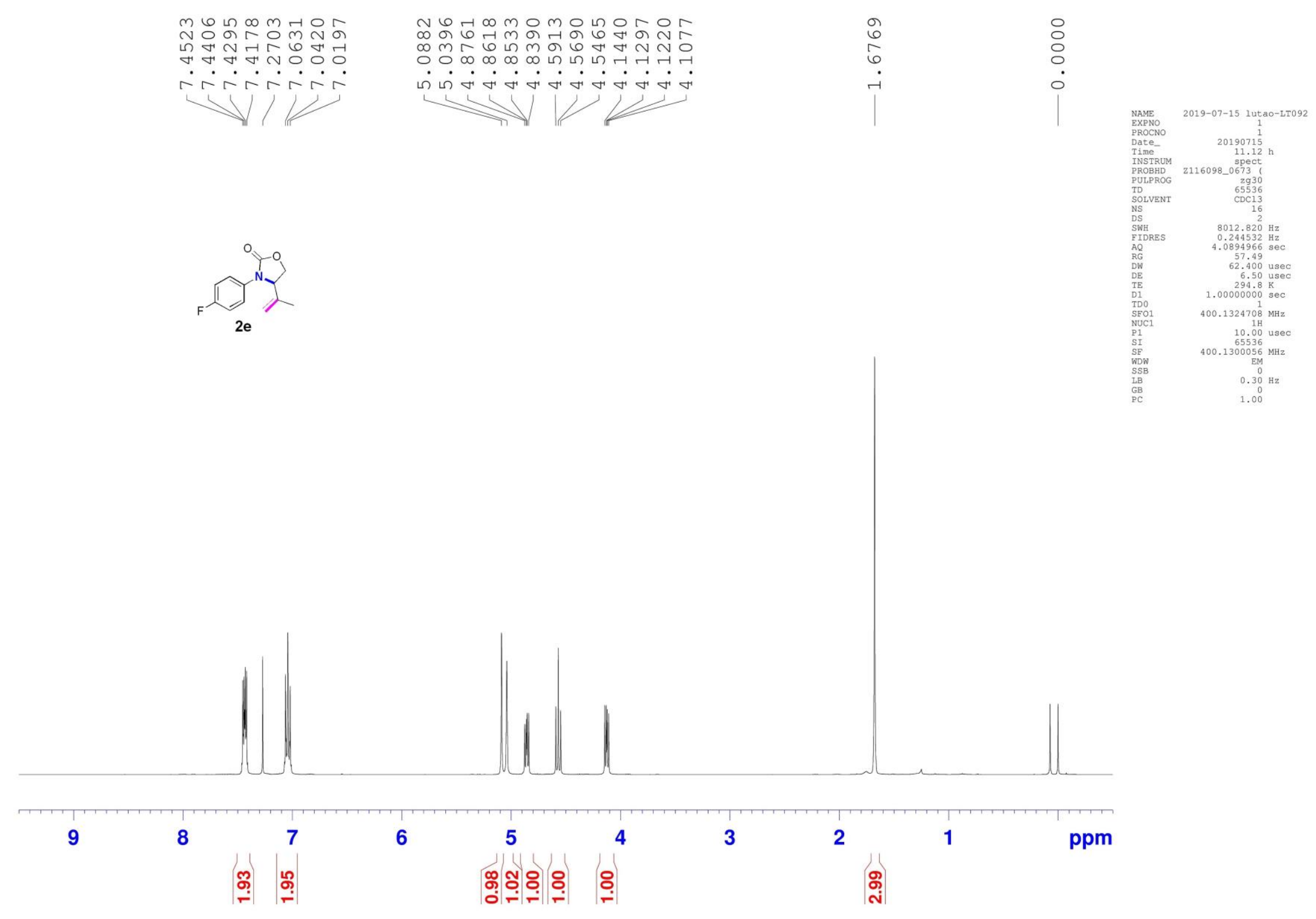




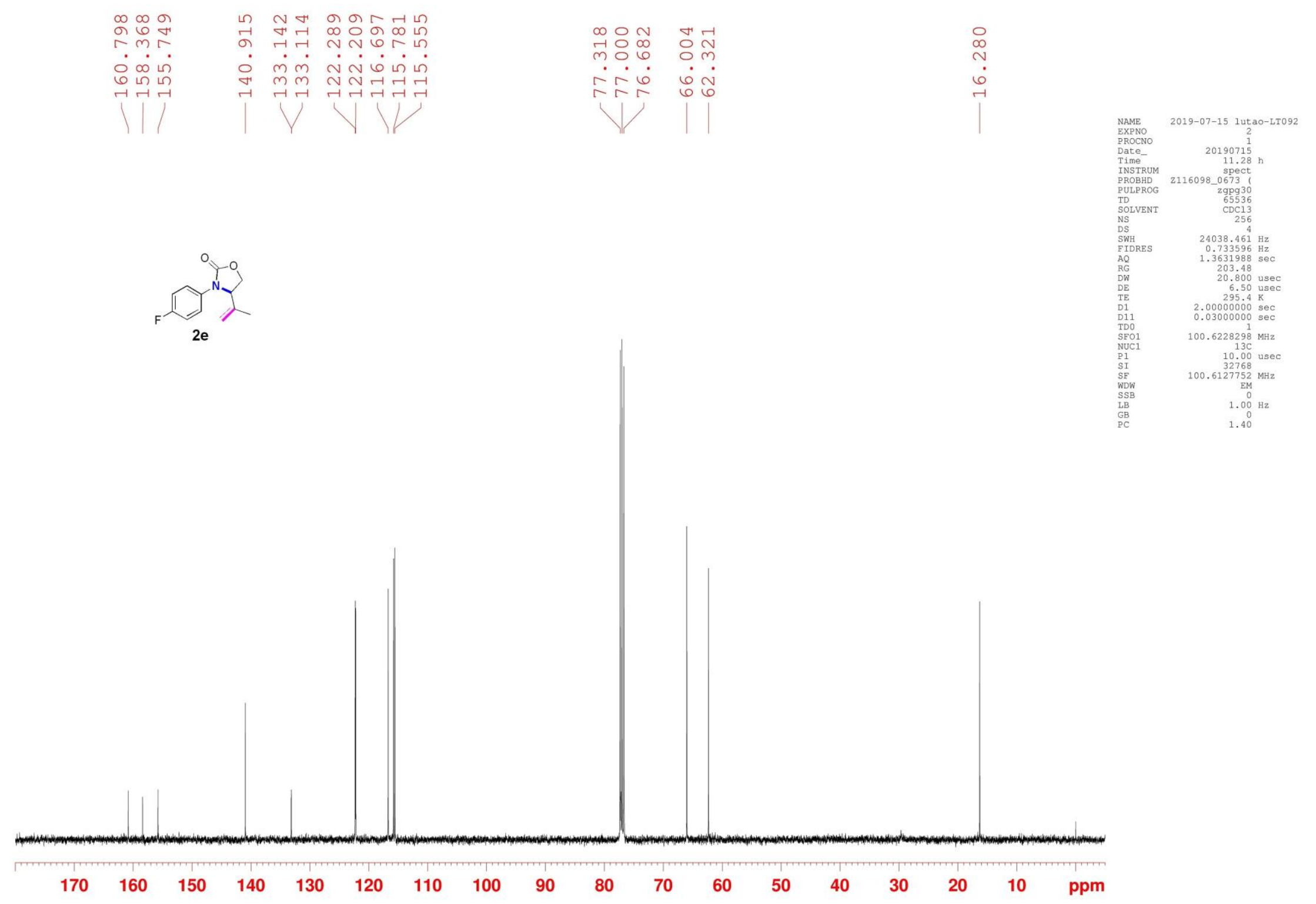




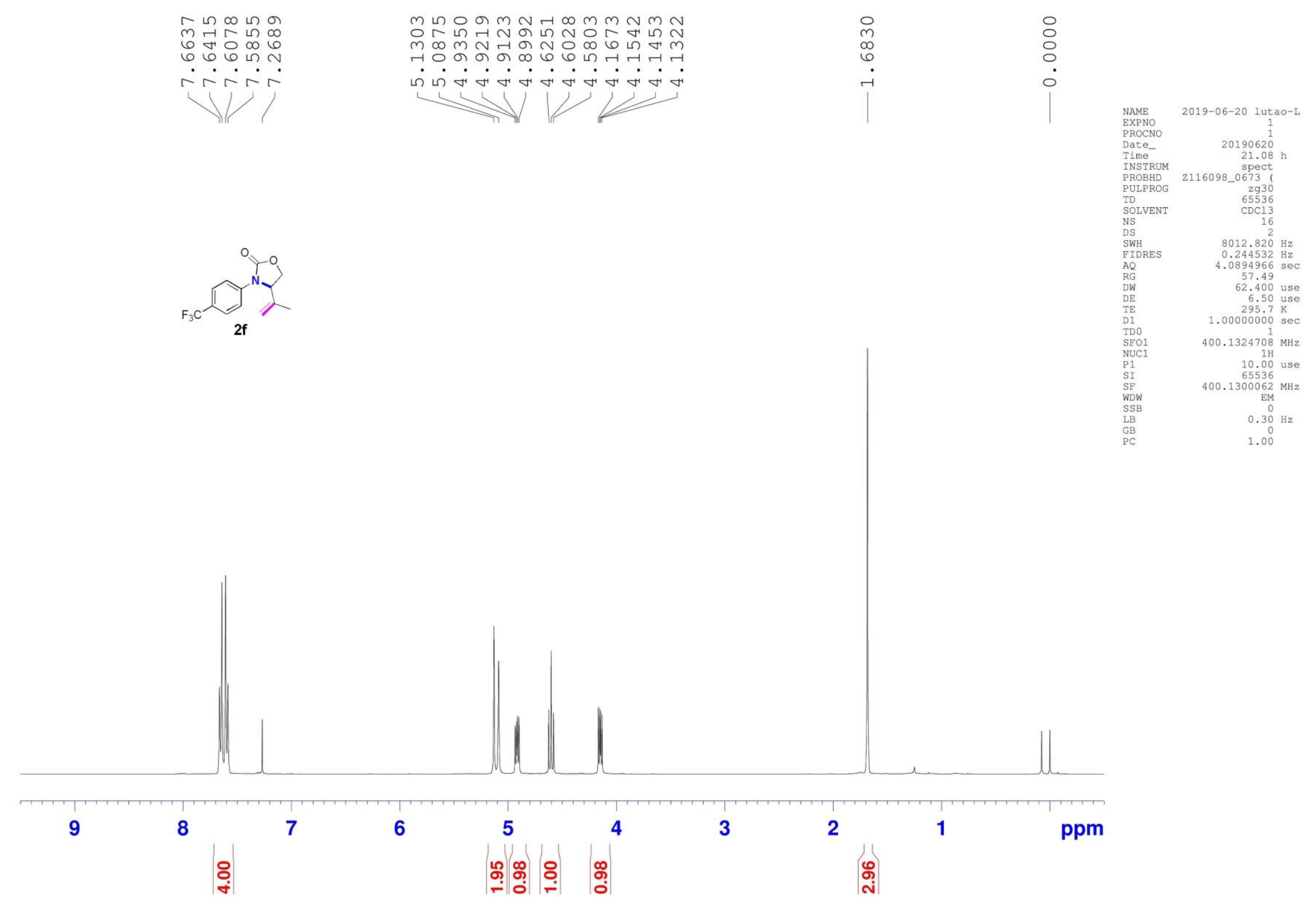




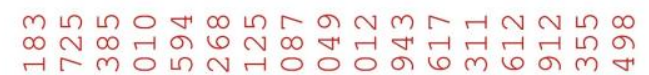

. $\cdot$.

மூ

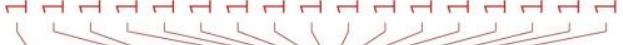

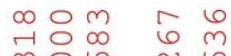

भ.र.

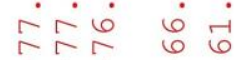

$\longrightarrow$
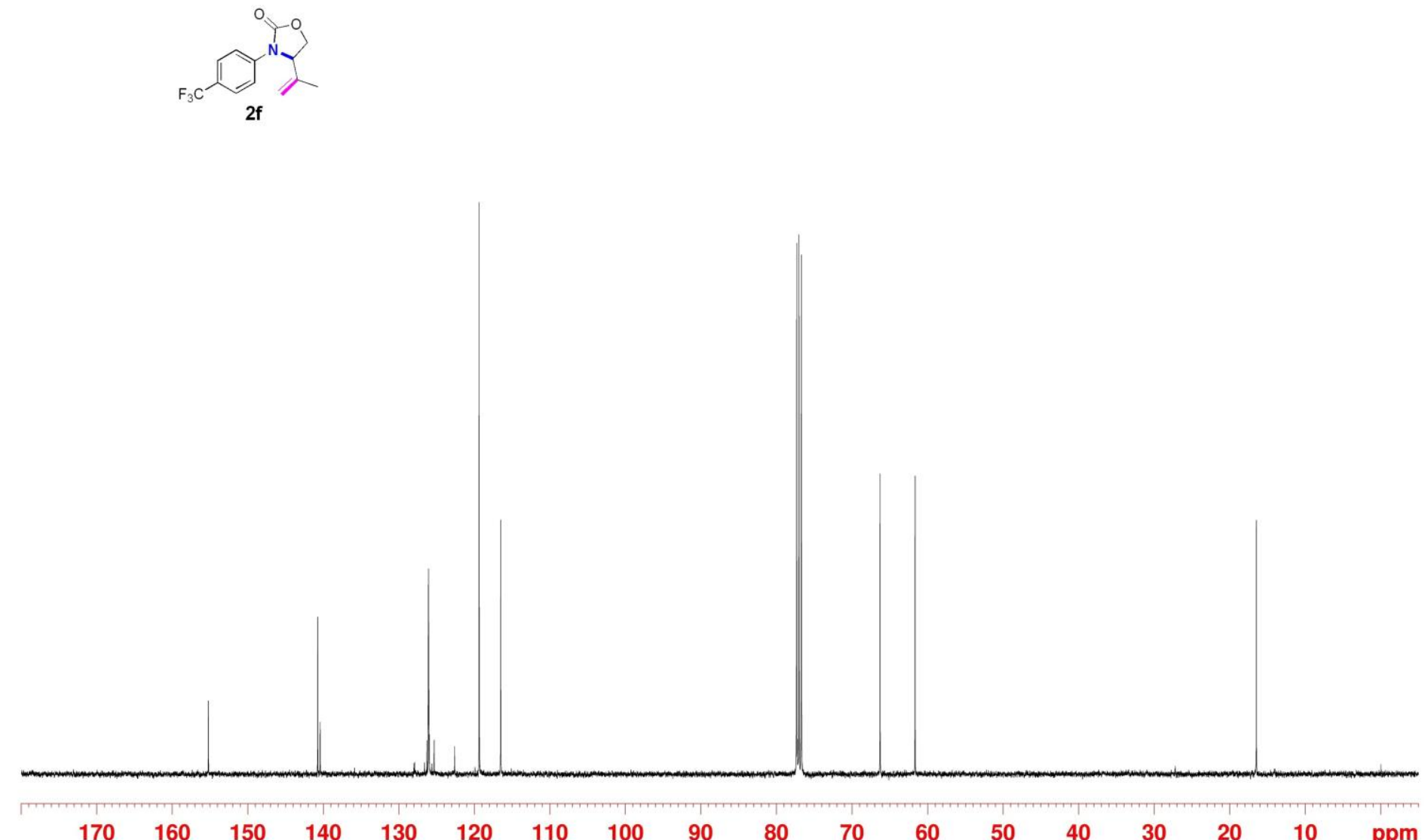


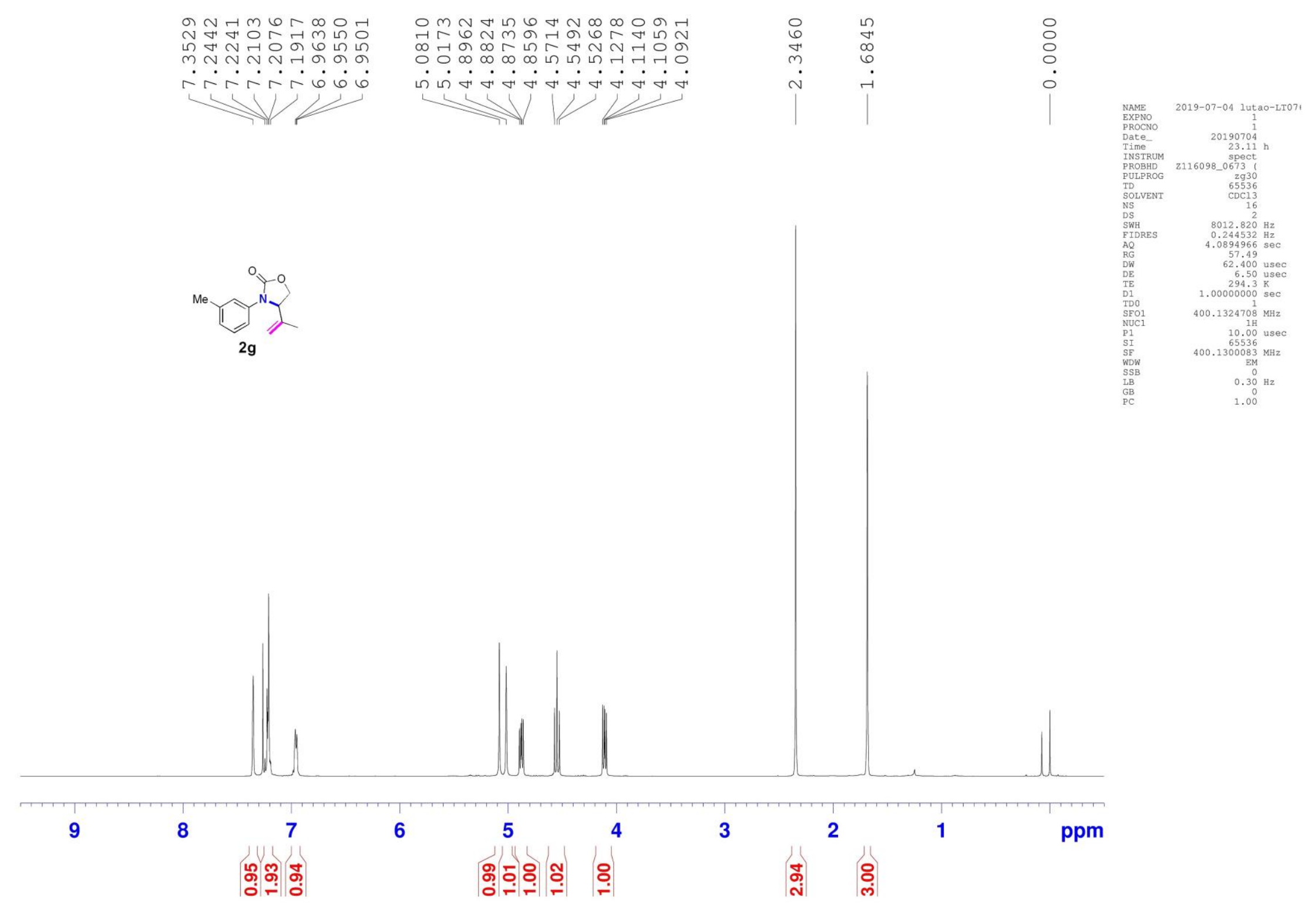



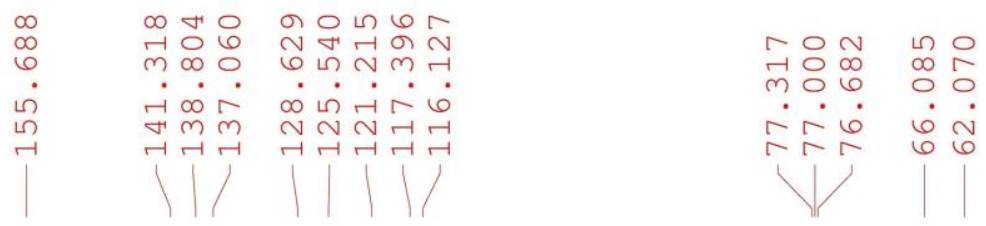

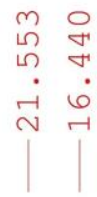
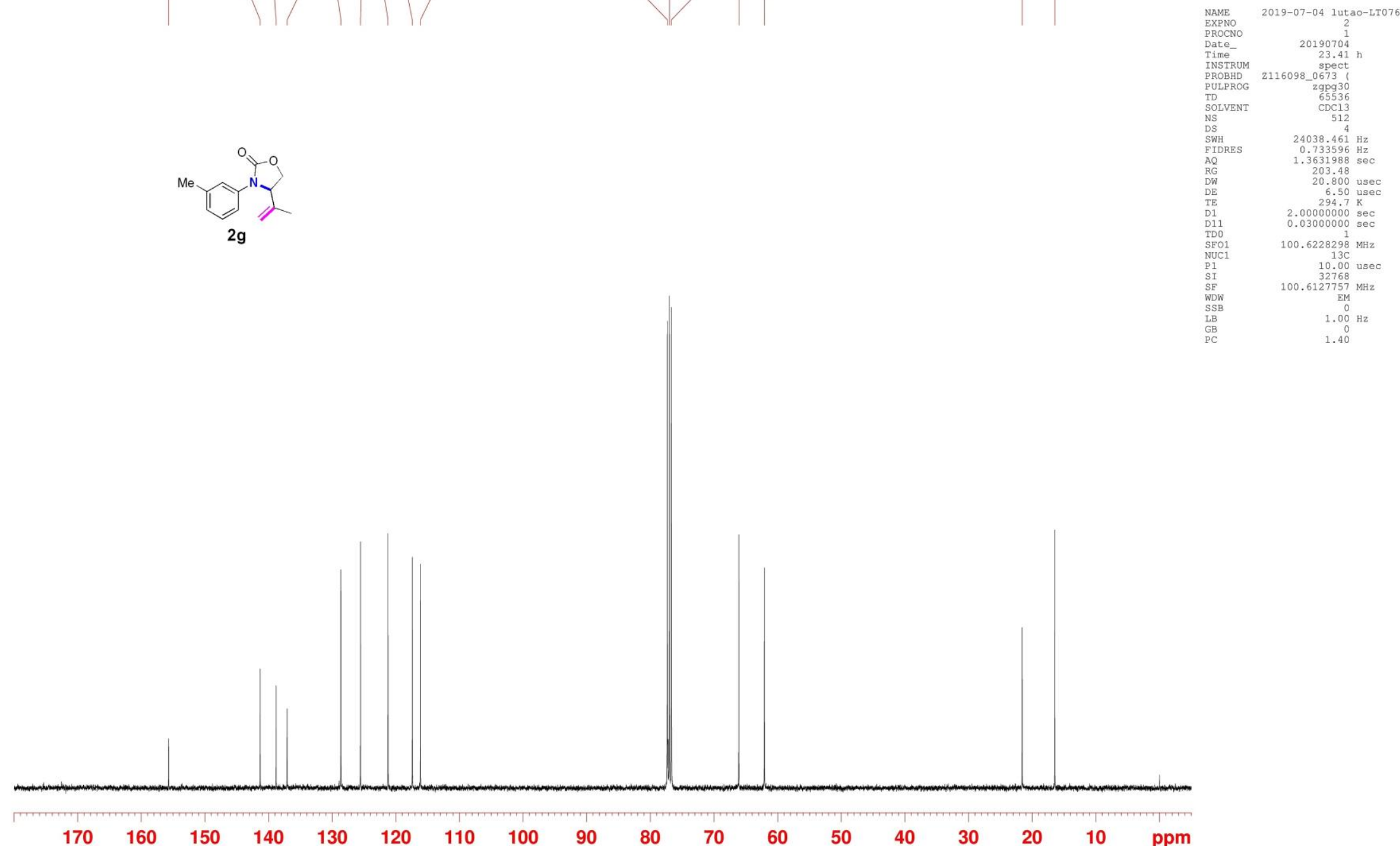


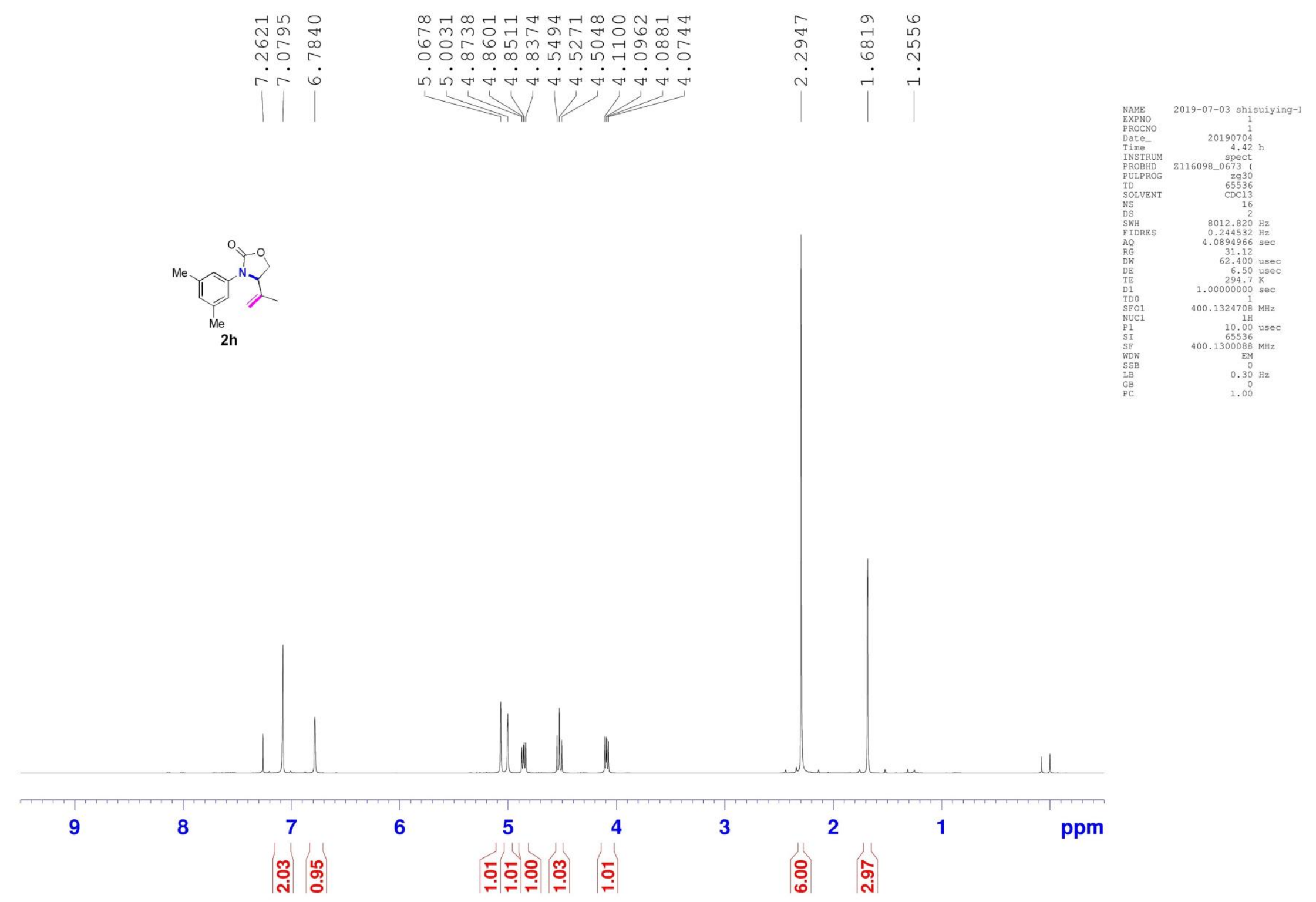




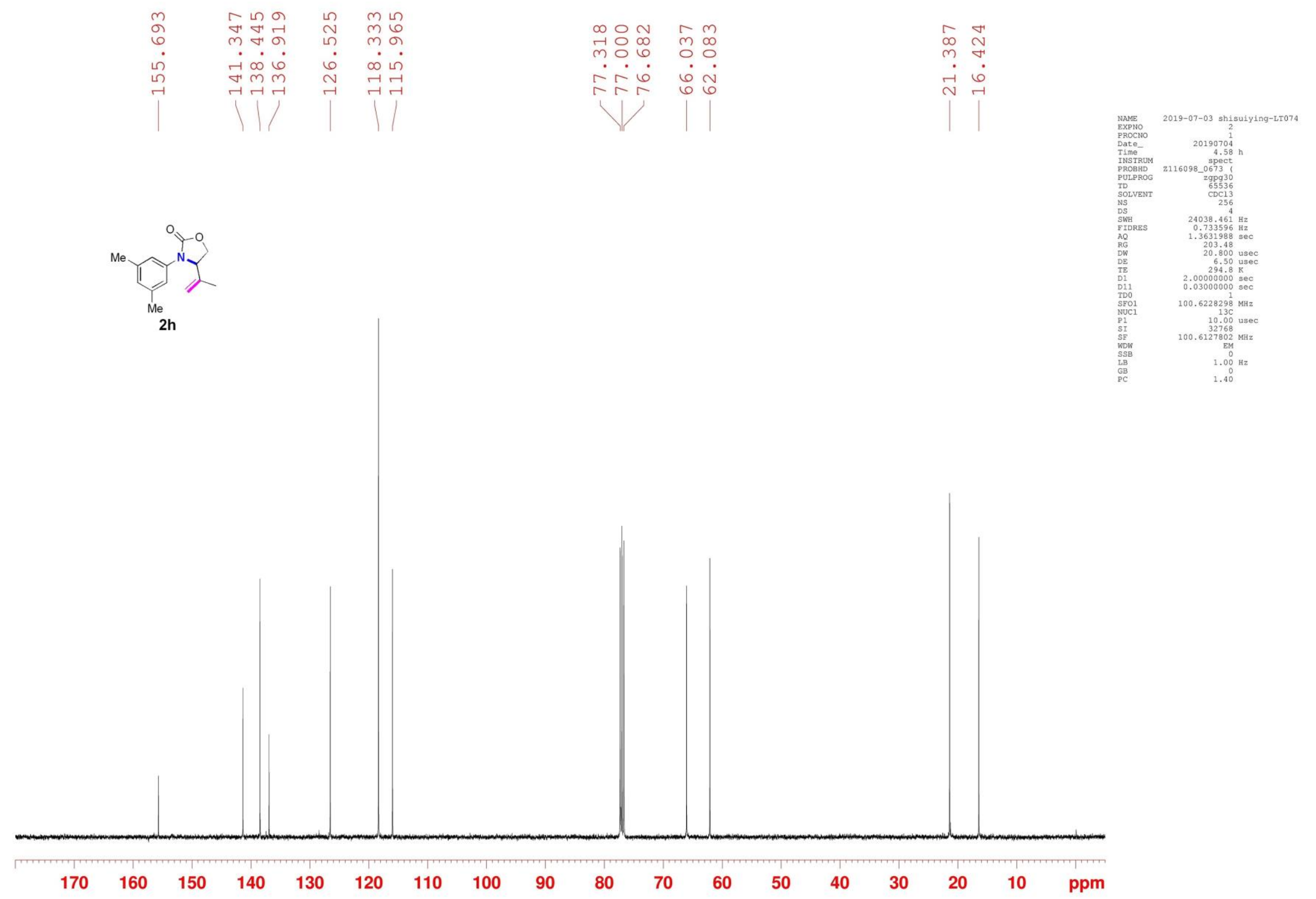




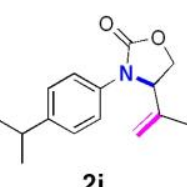

2i
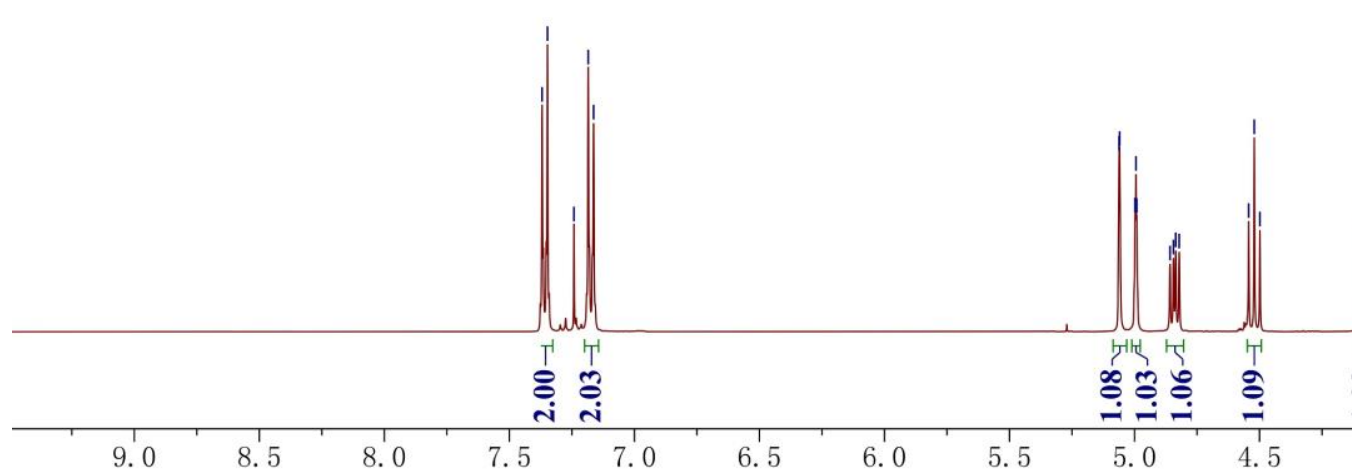

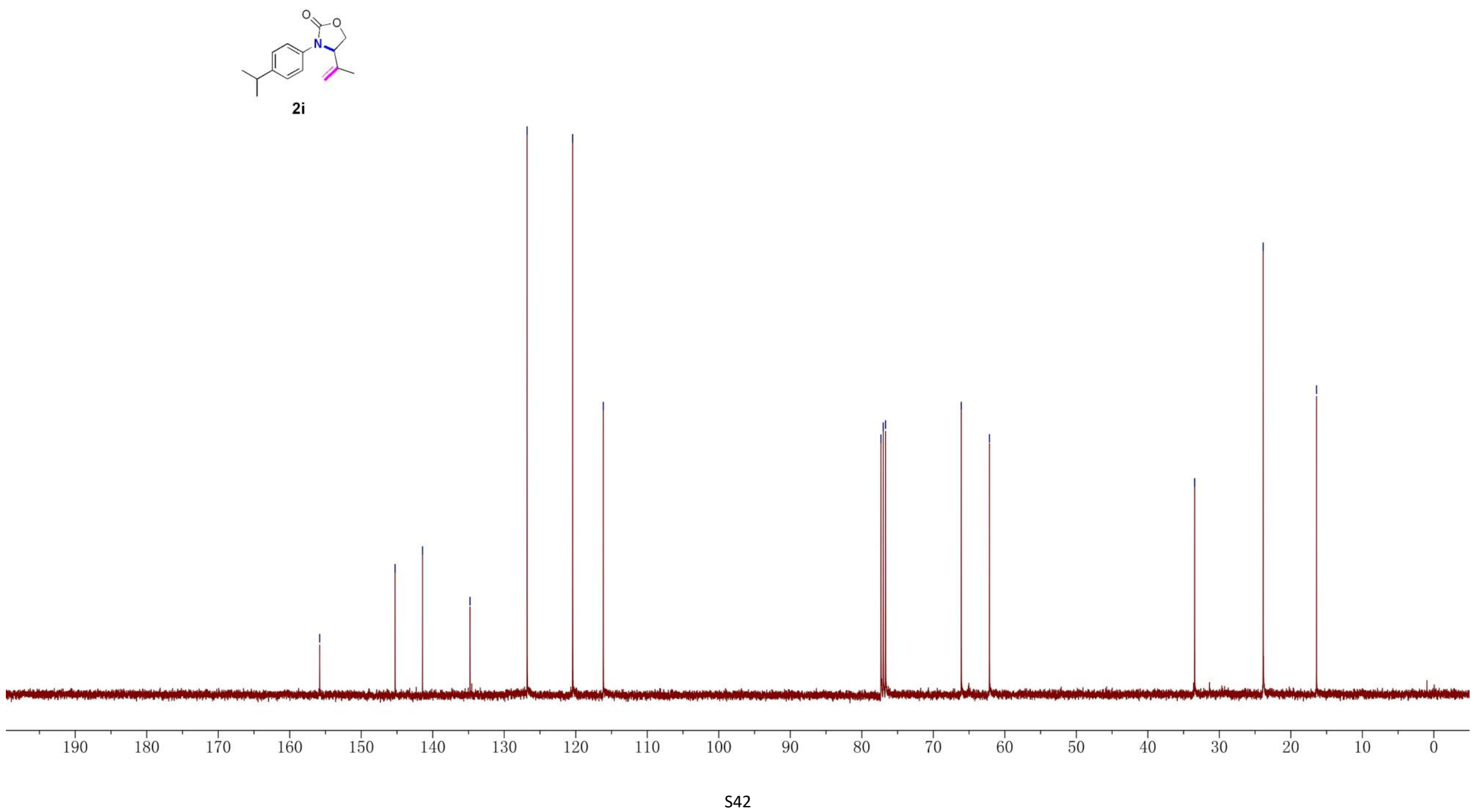


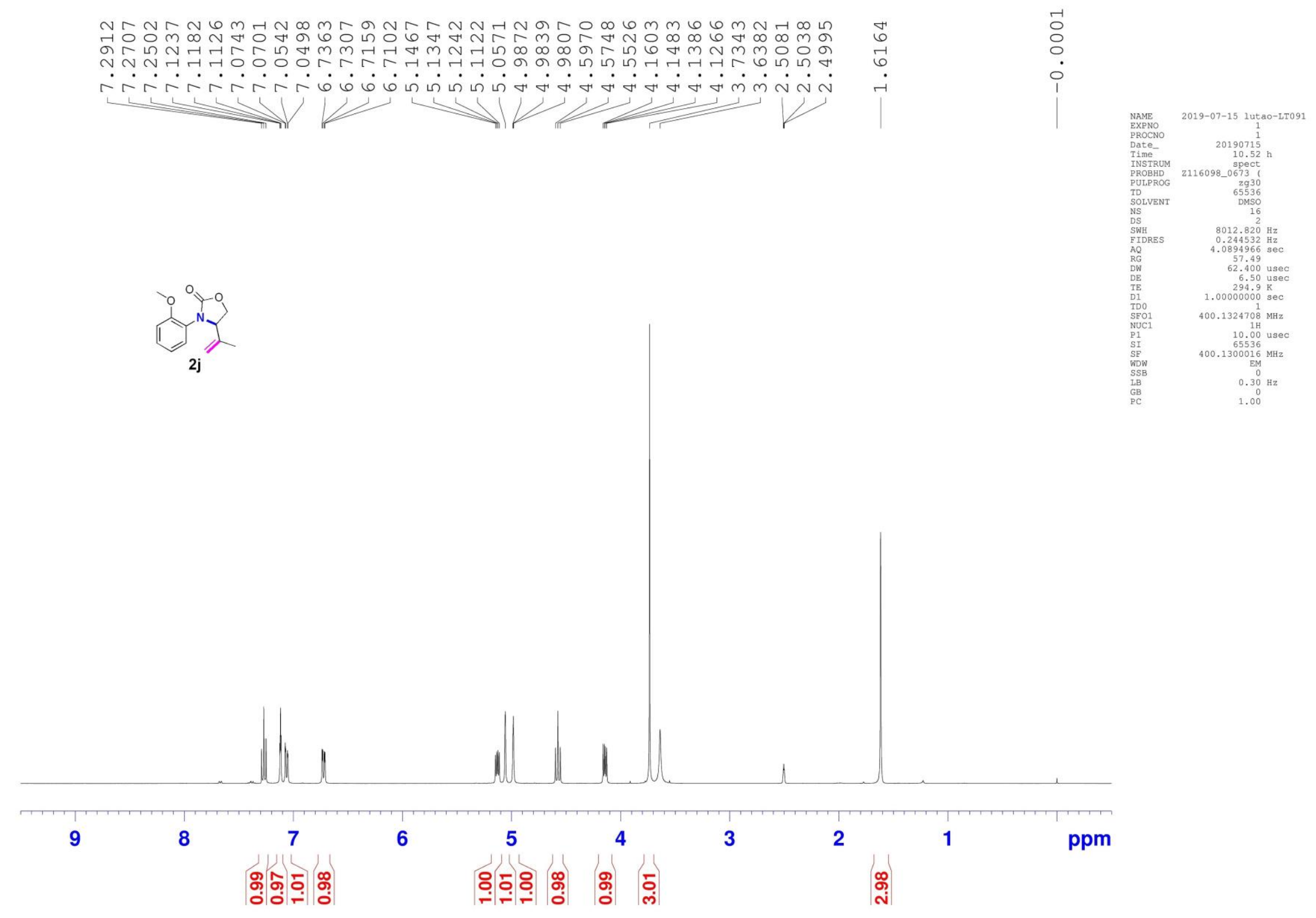




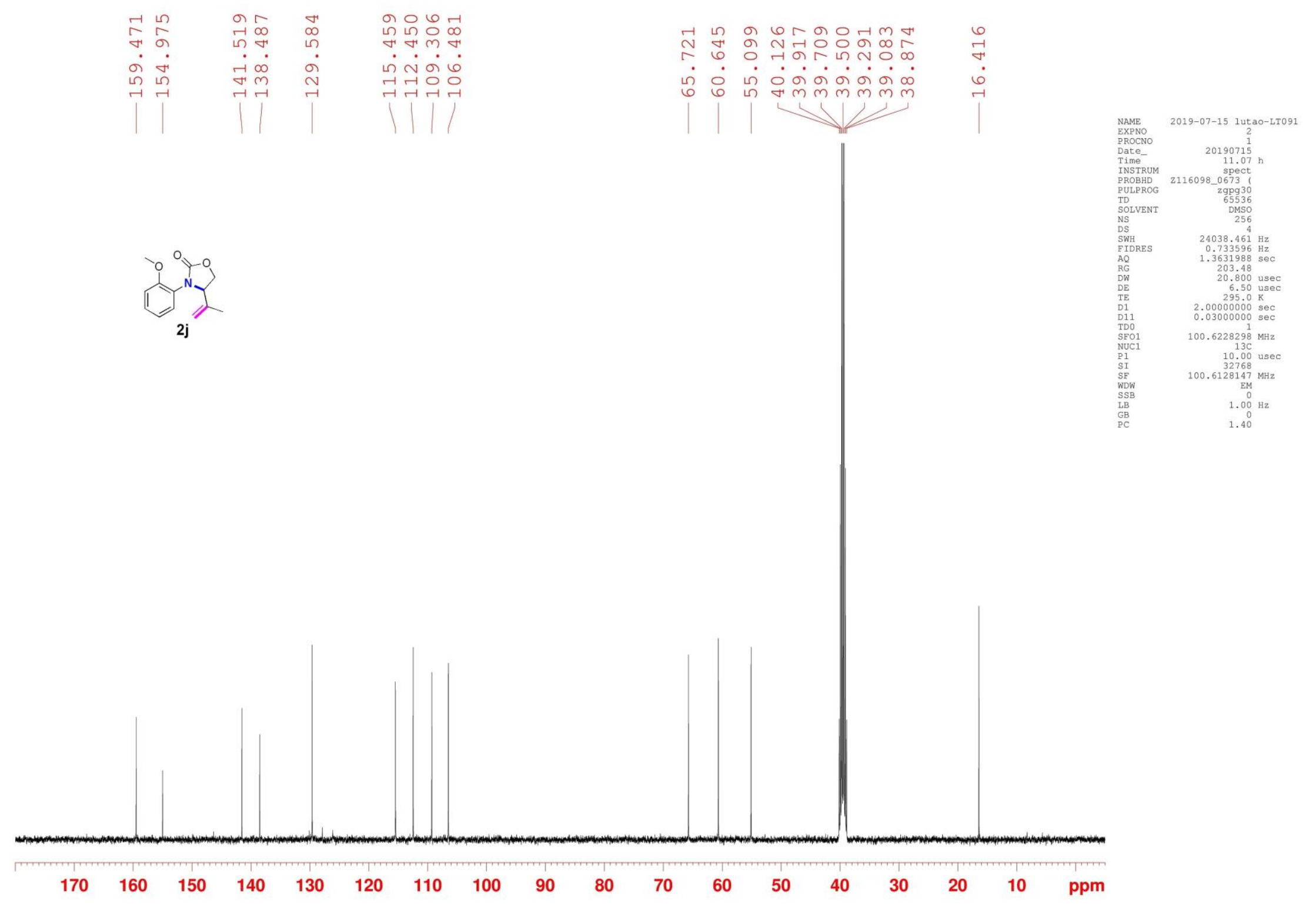




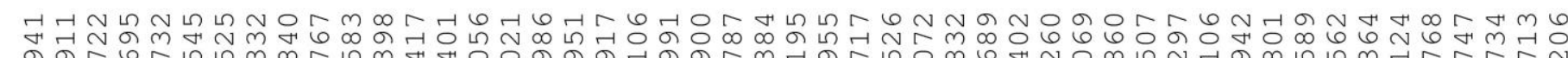

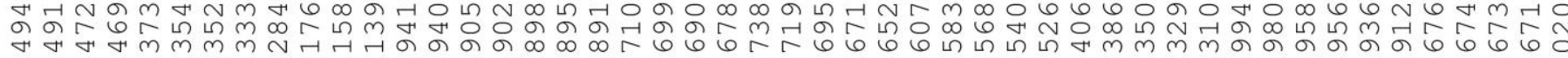
-

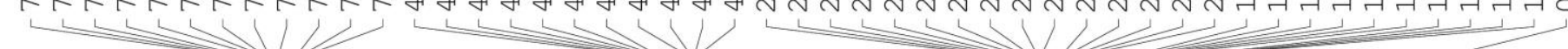
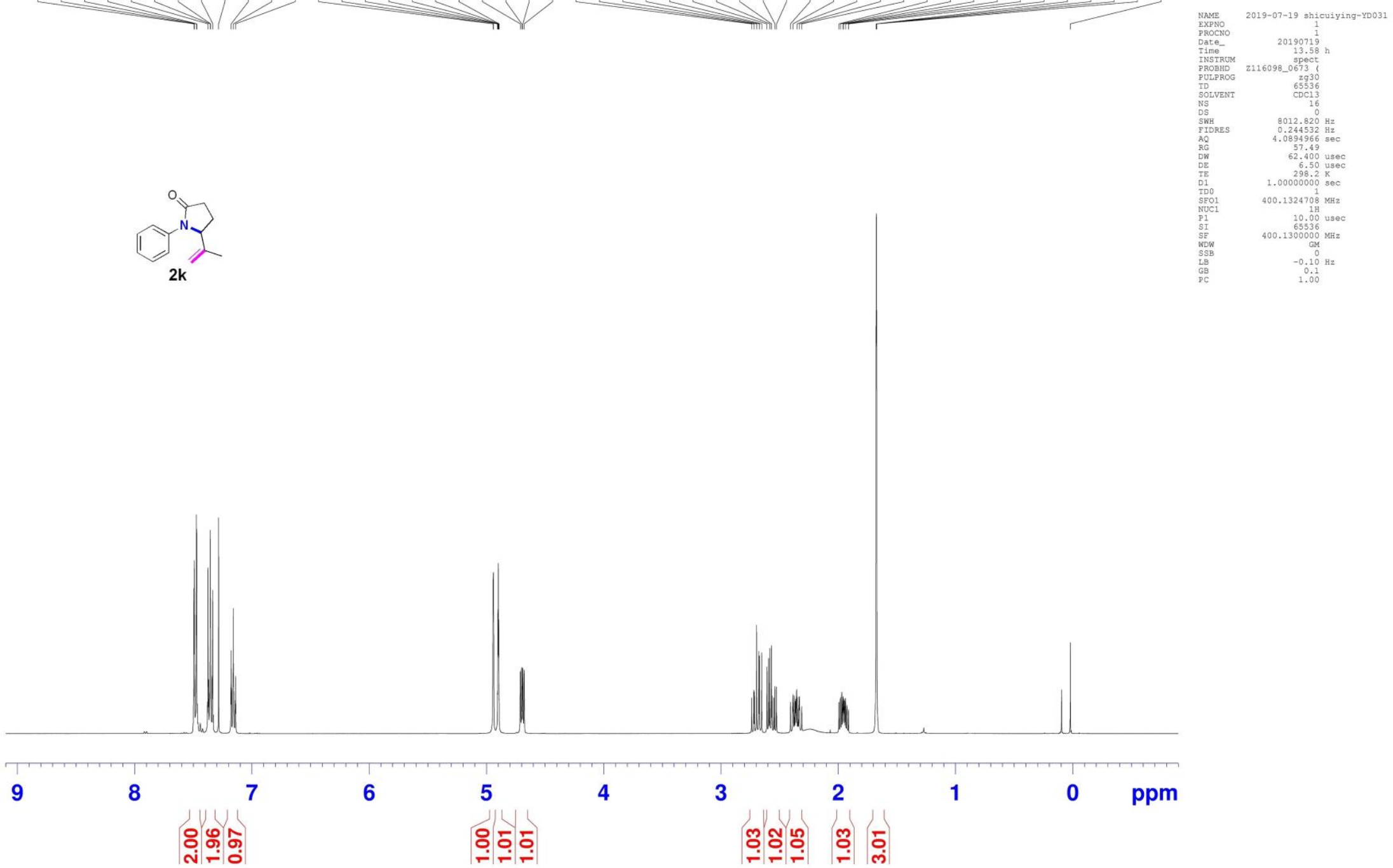


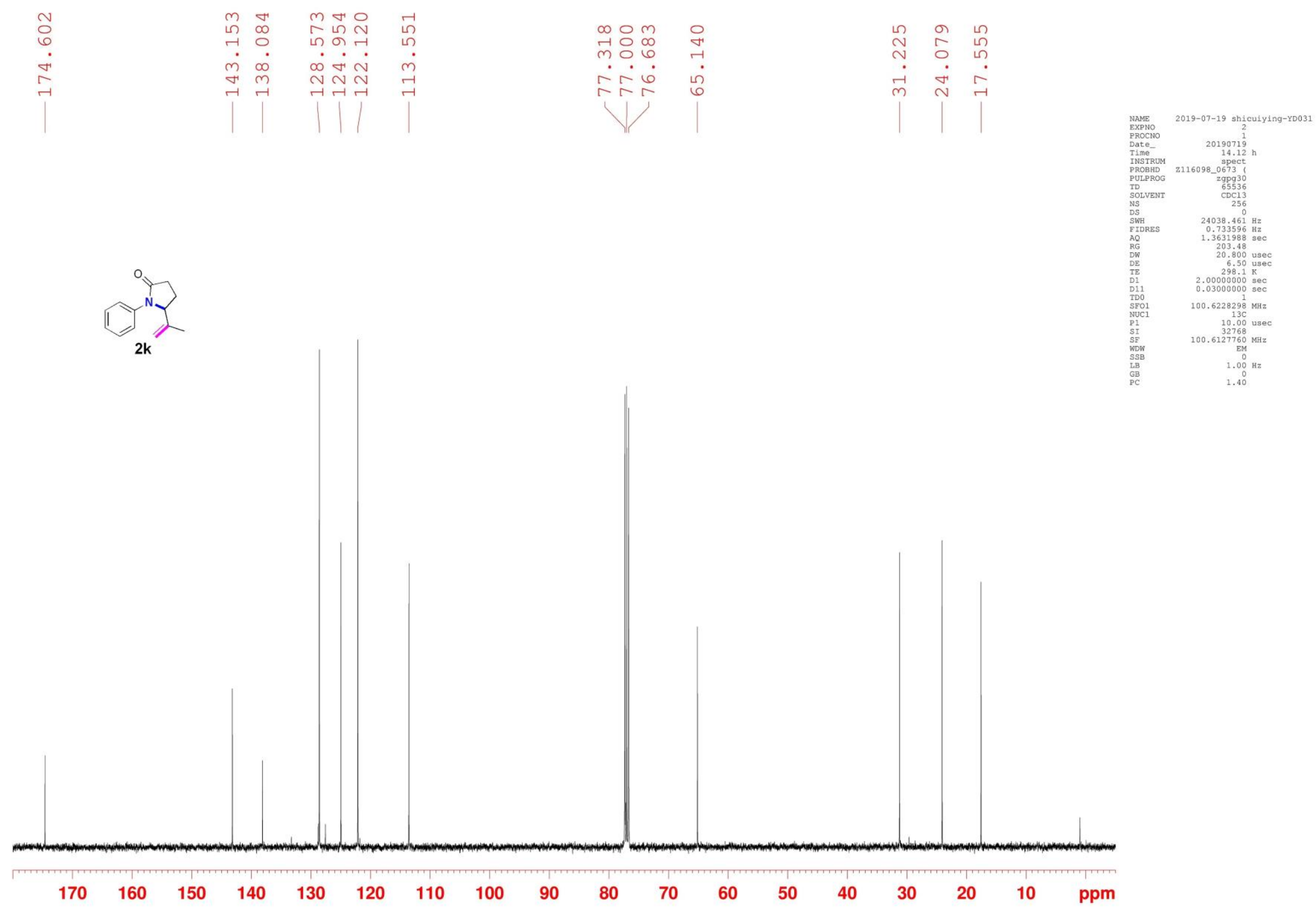




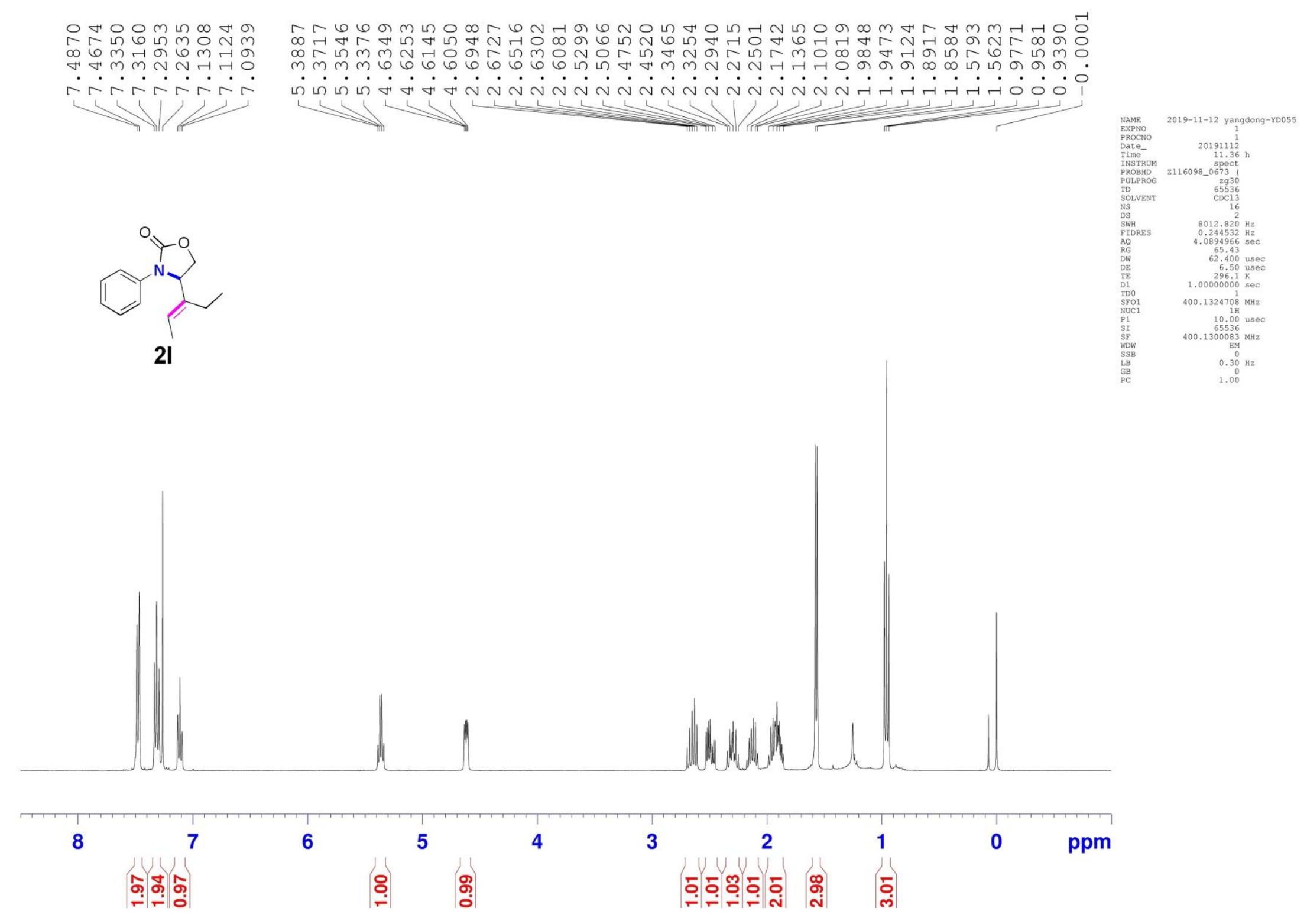




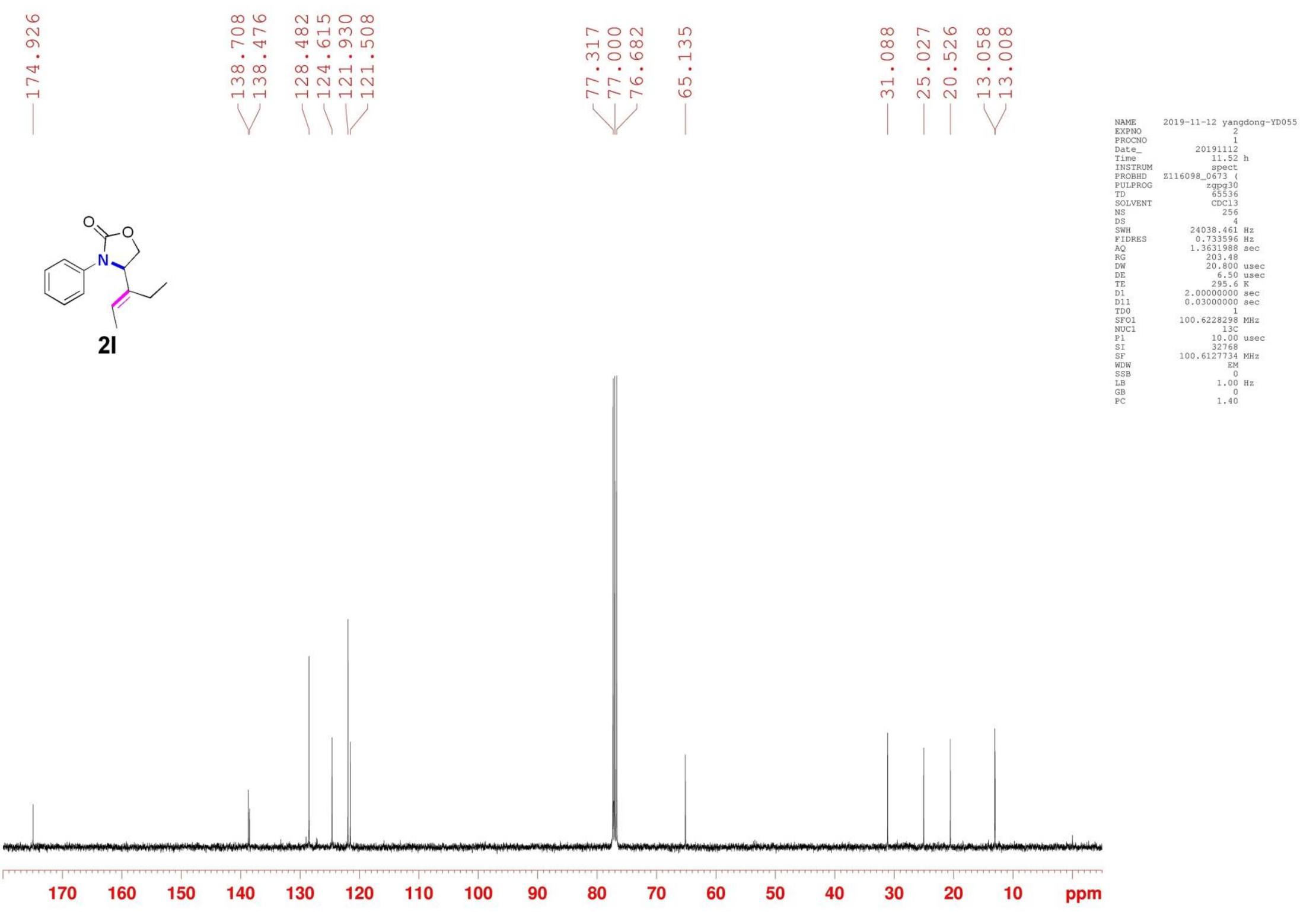




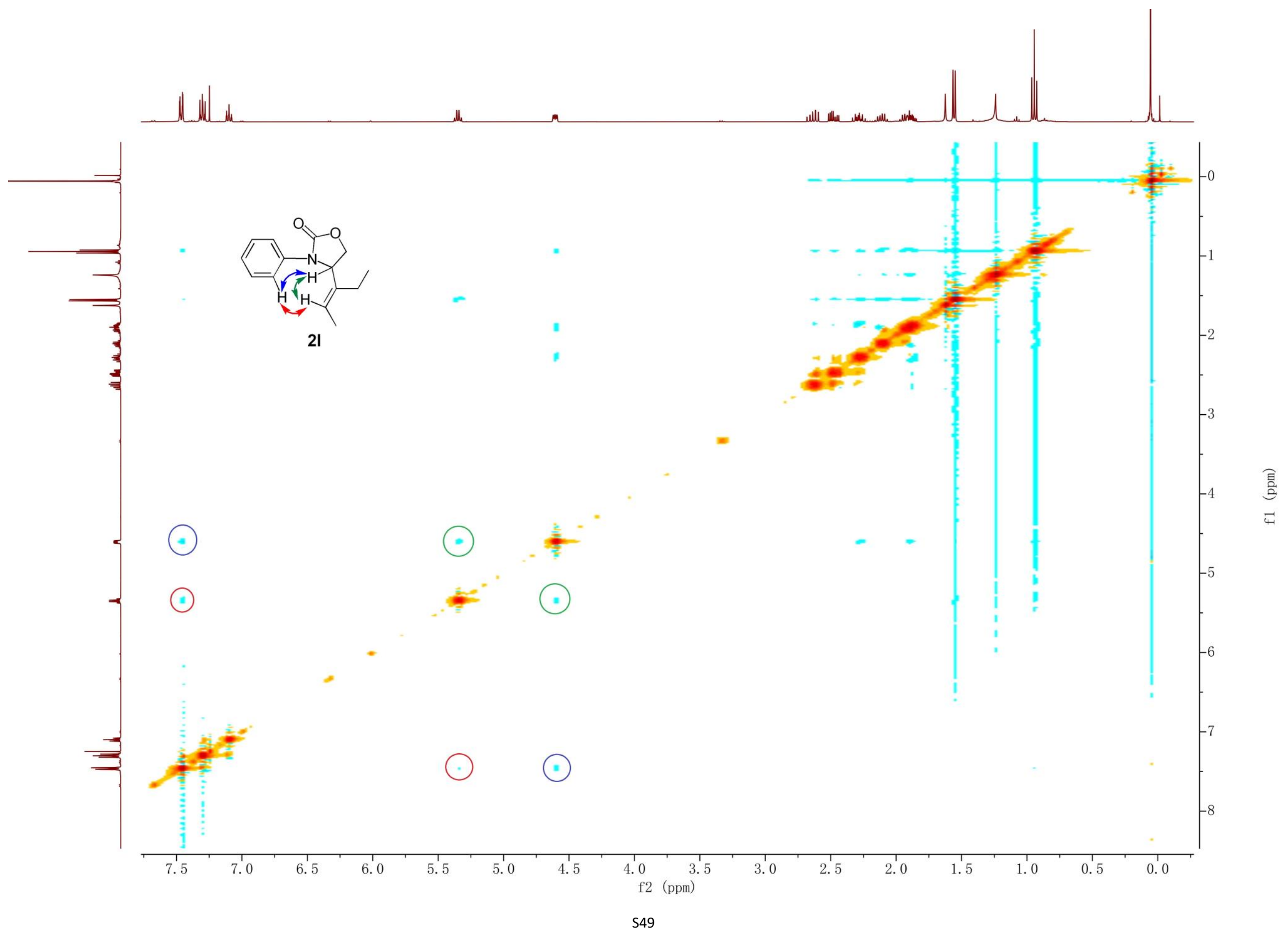




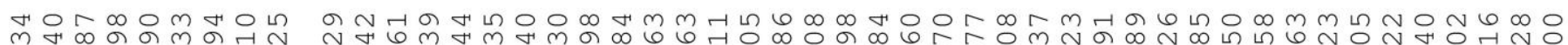

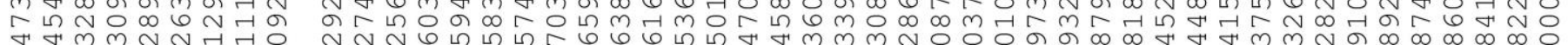

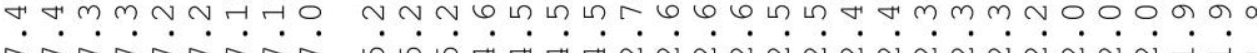

1

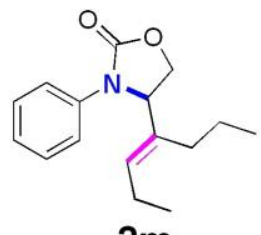

$2 \mathrm{~m}$
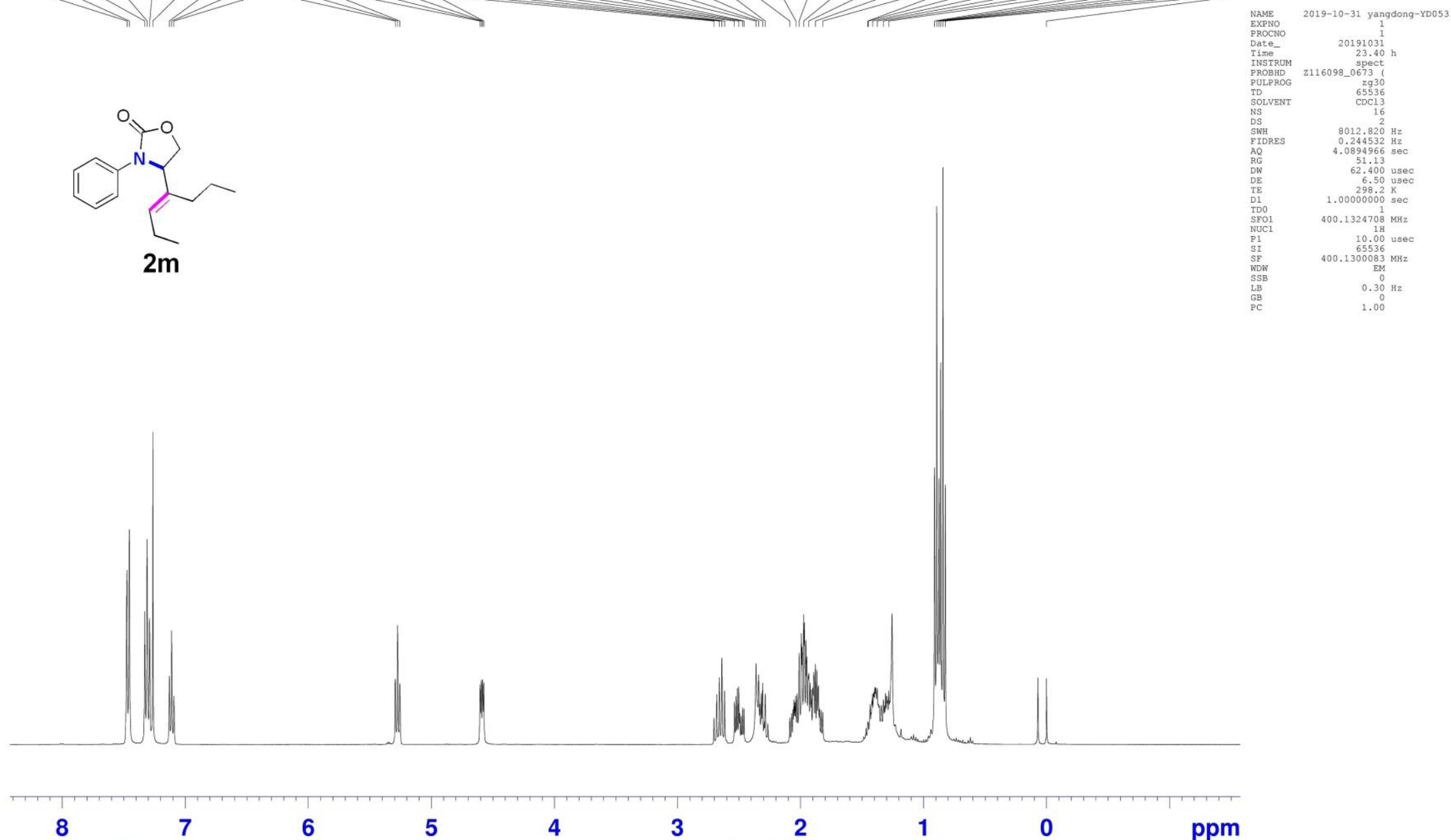

6

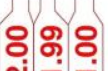

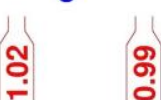

3
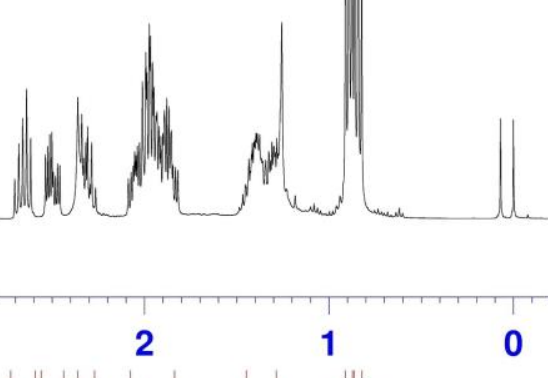

ppm

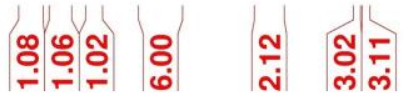



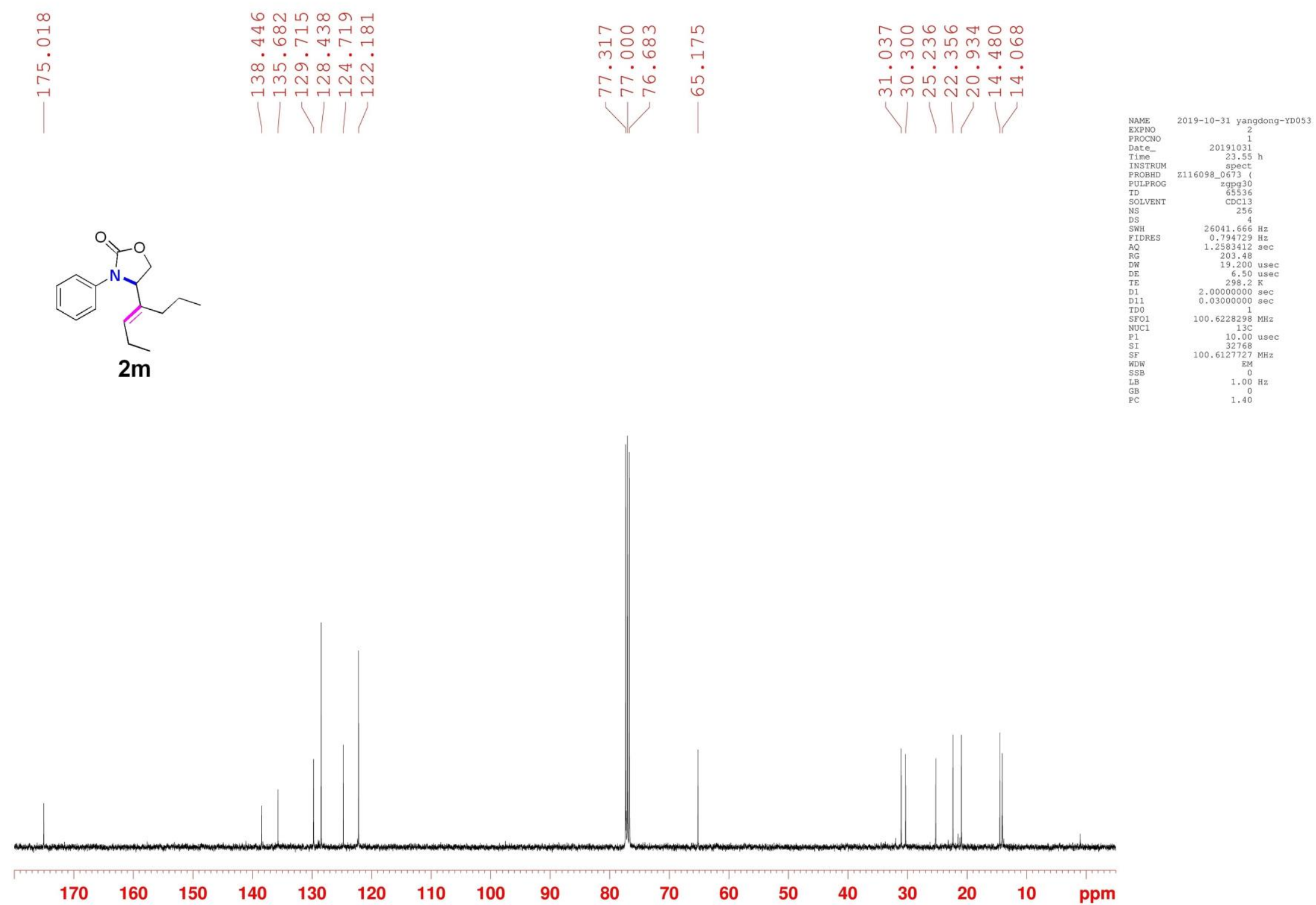


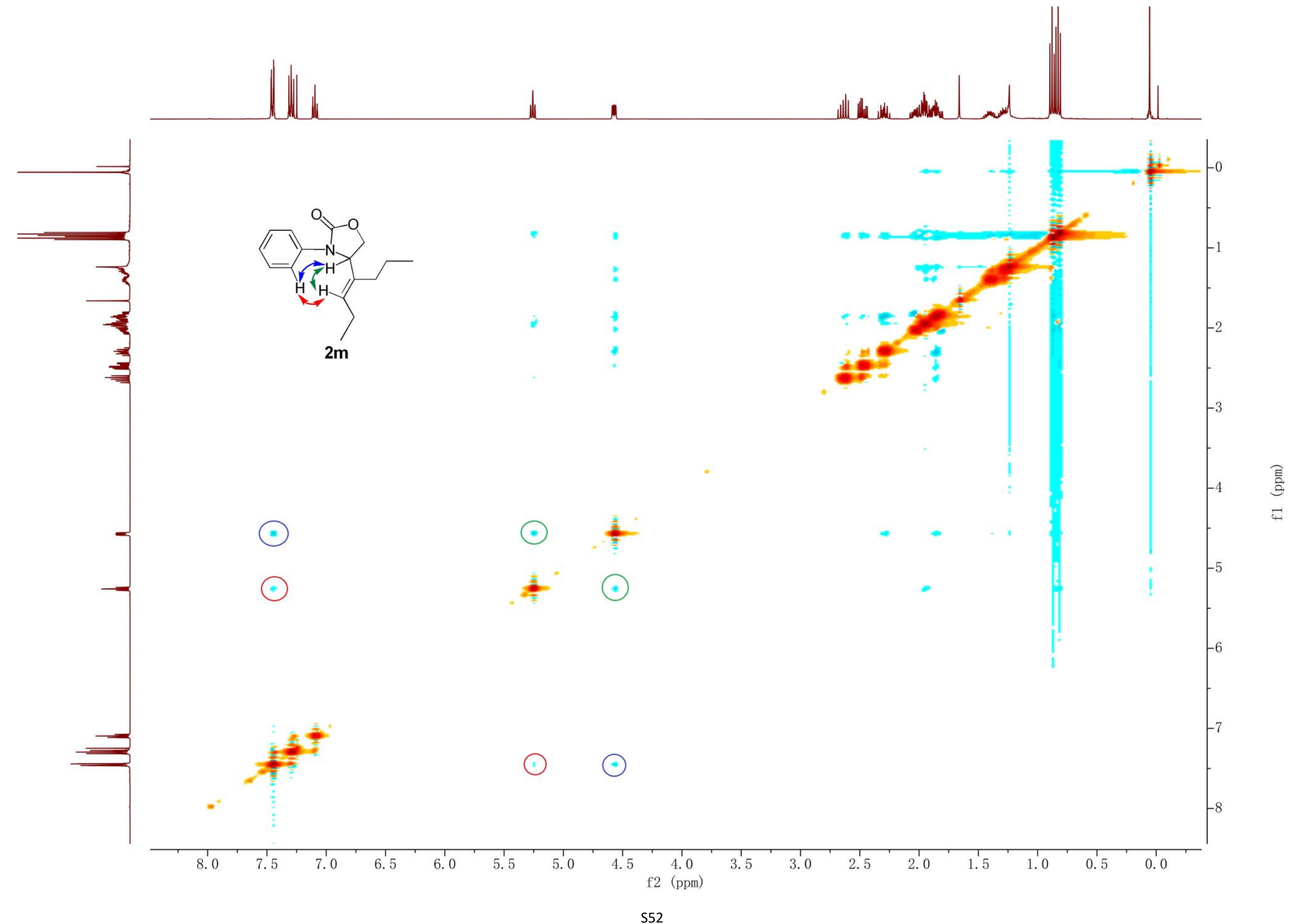



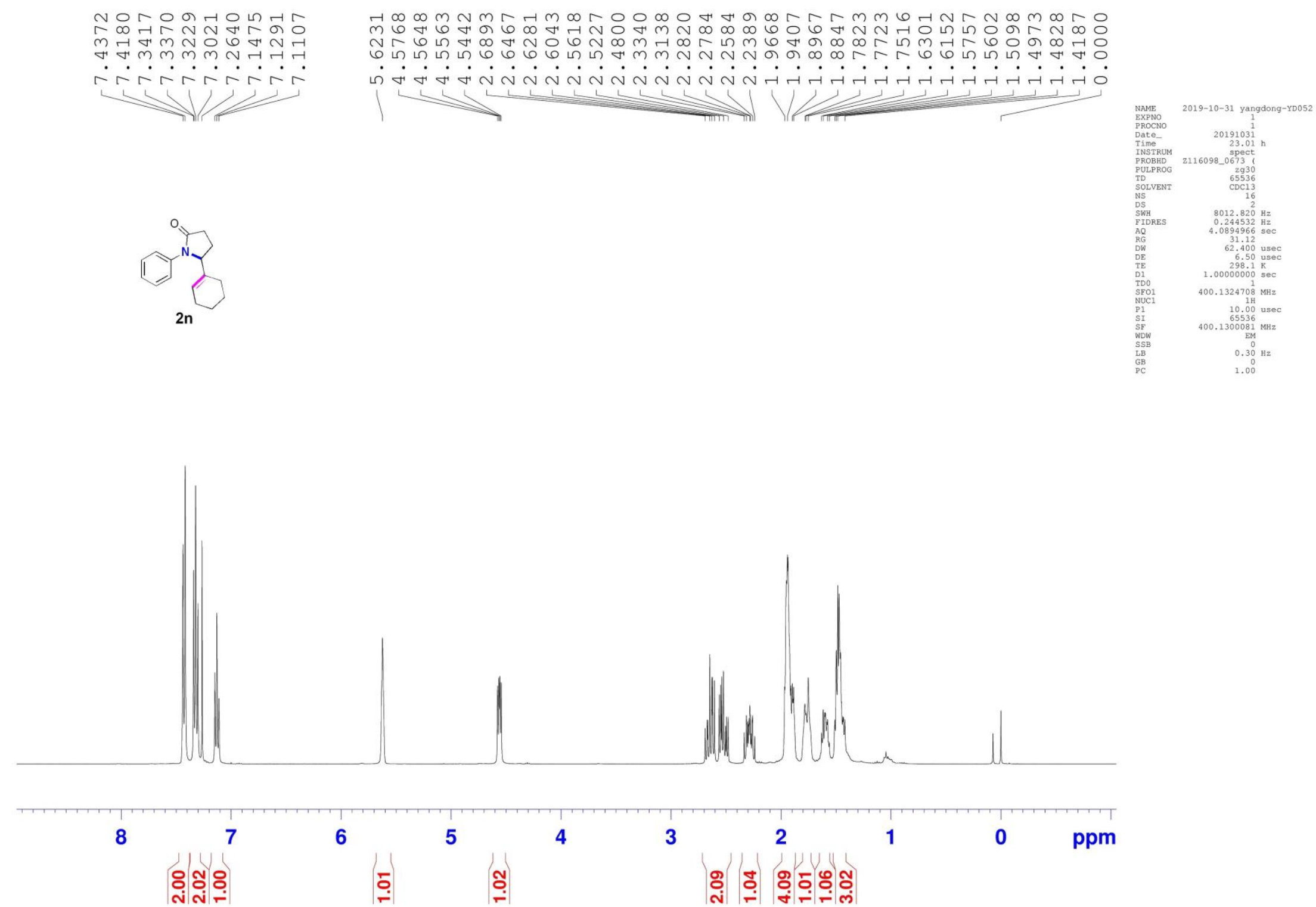


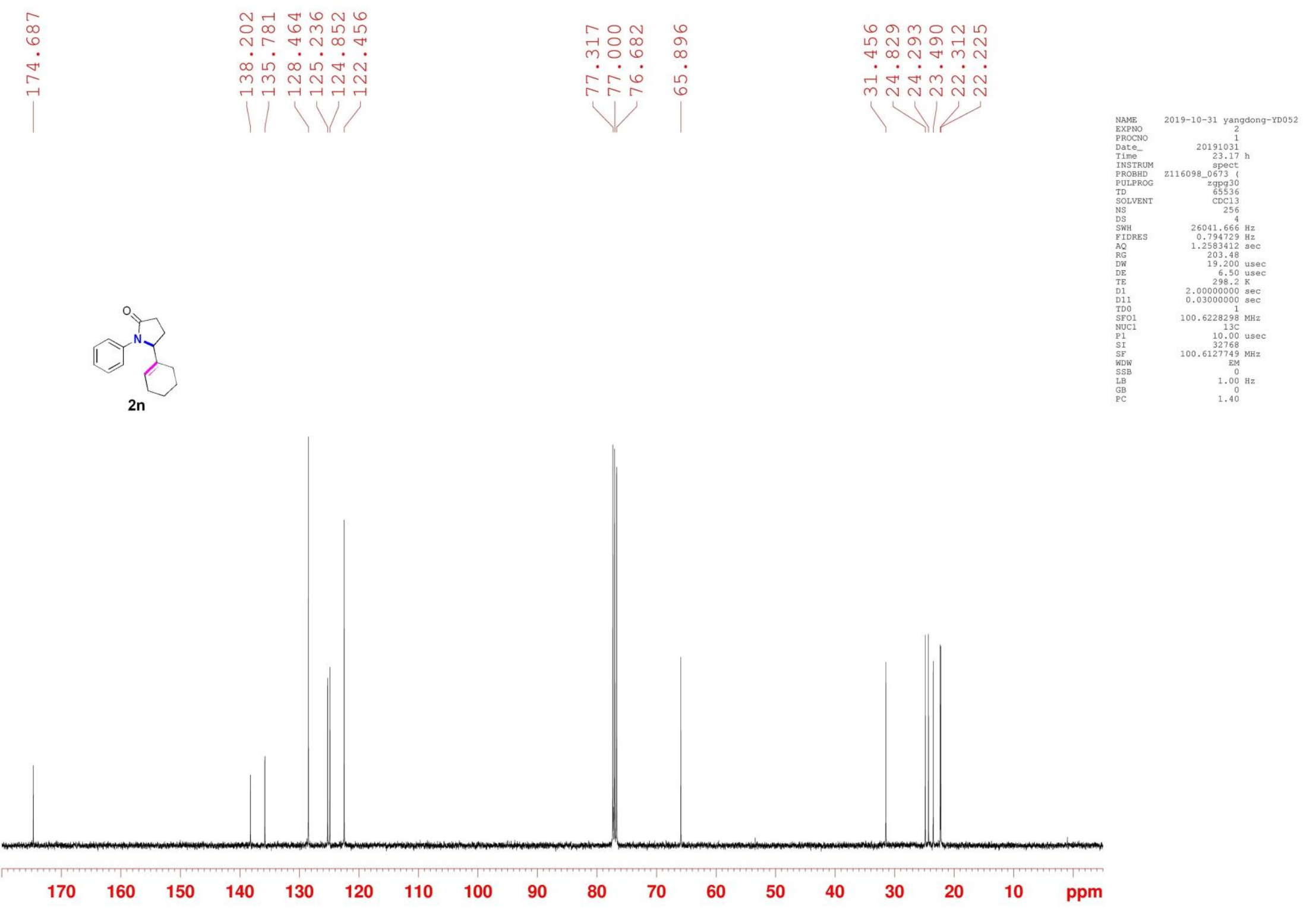



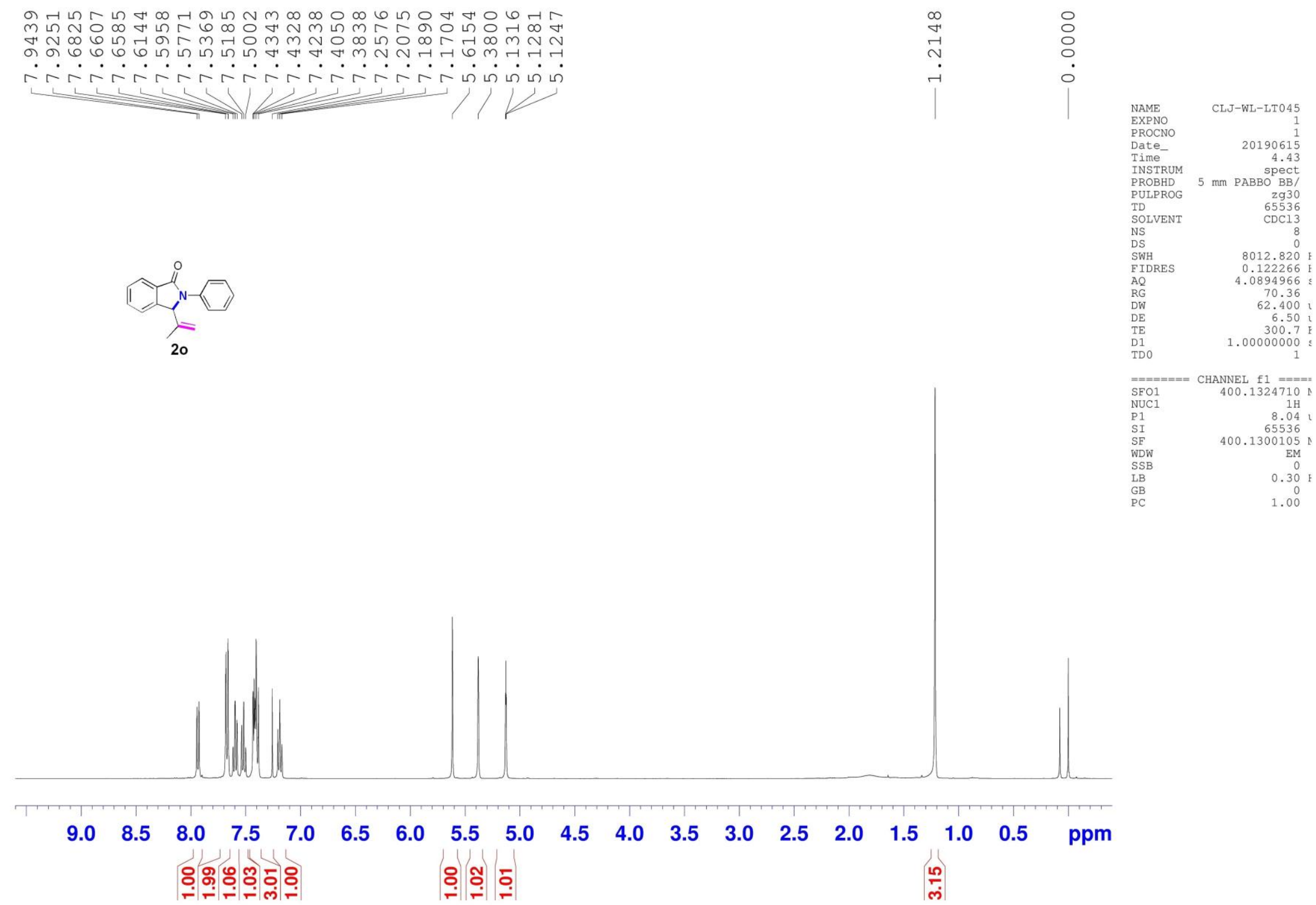


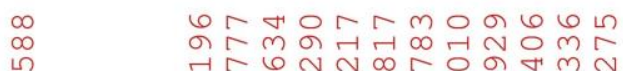

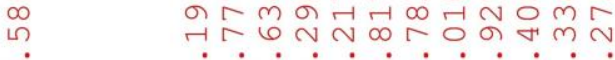

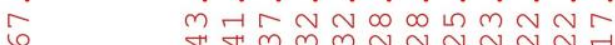

가다다각ㄱㄱㄱㄱ

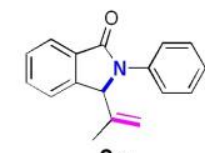

$\infty \circ \infty$

in

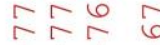
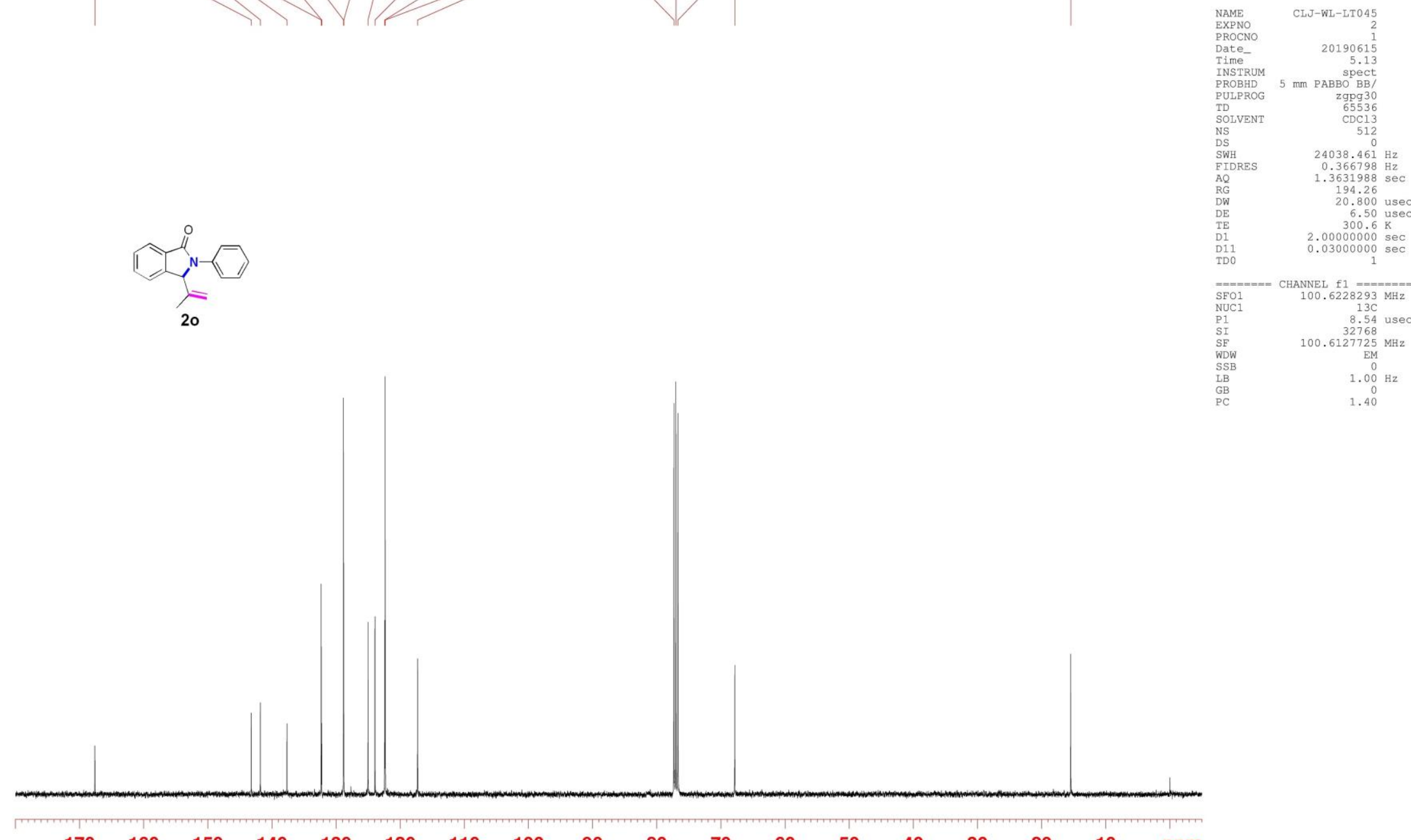
170160
$\begin{array}{lll}140 & 130\end{array}$
$\begin{array}{llll}120 & 110 & 100 & 90\end{array}$
$\mathbf{3 0}$
20
ppm

60 


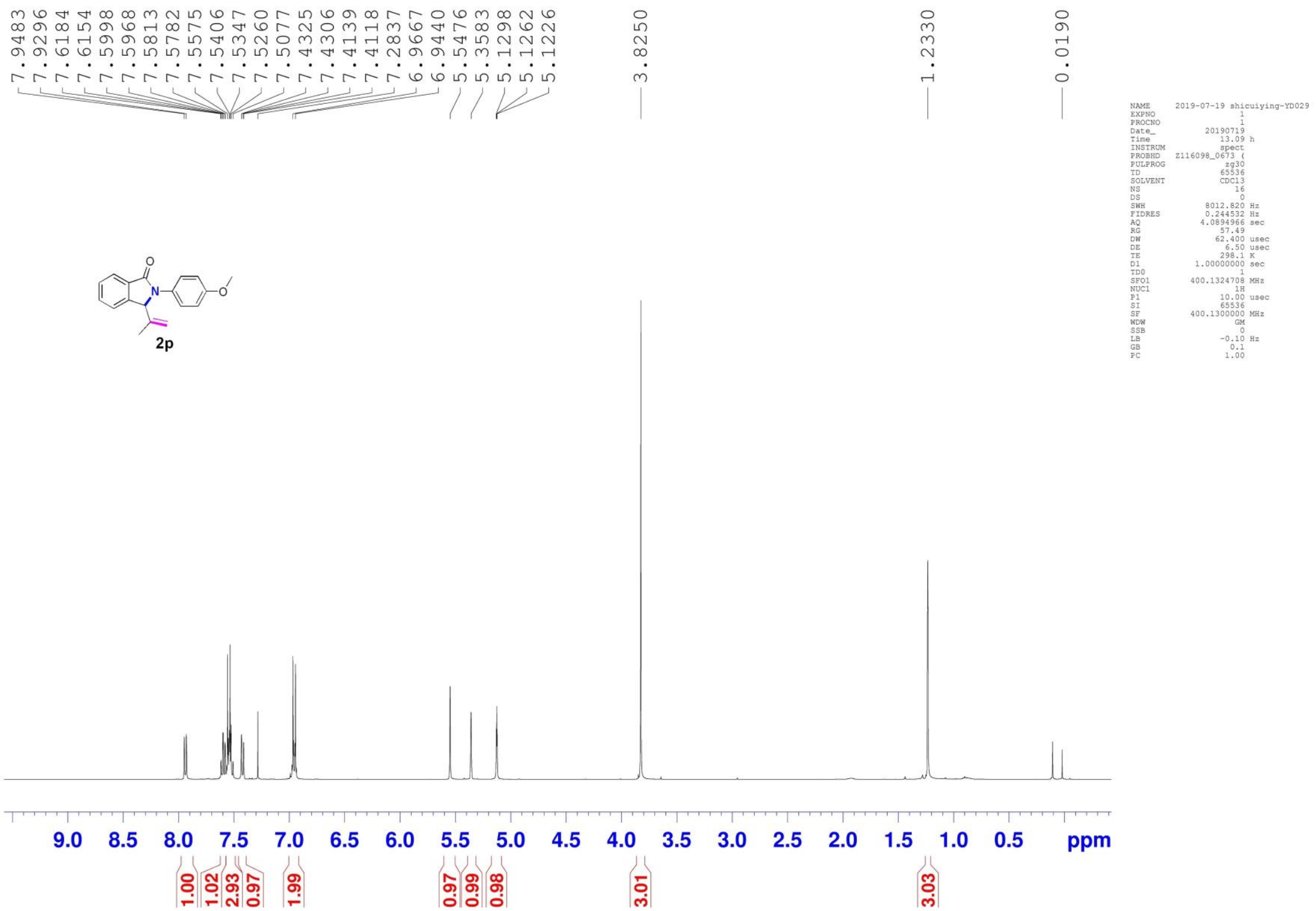




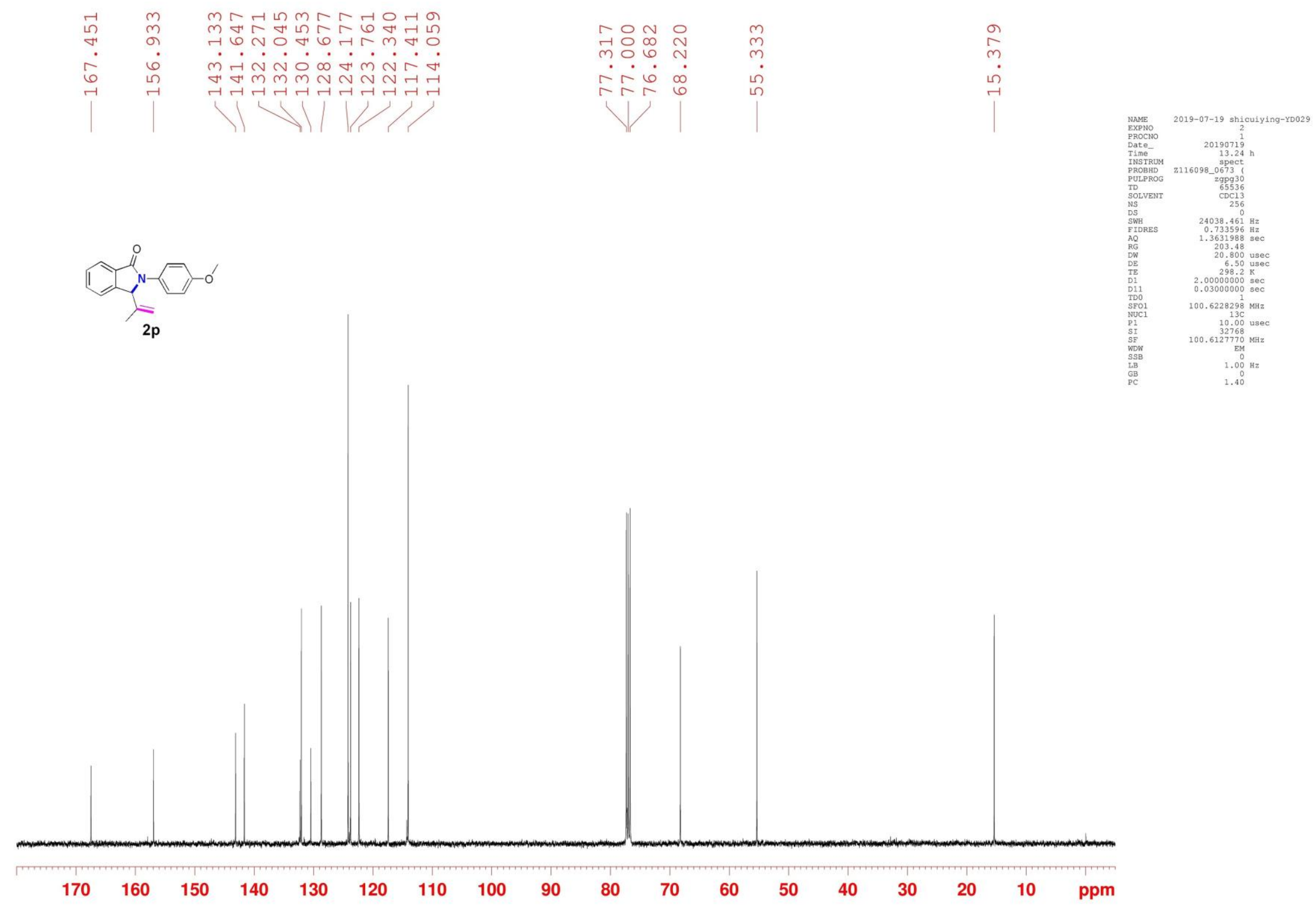



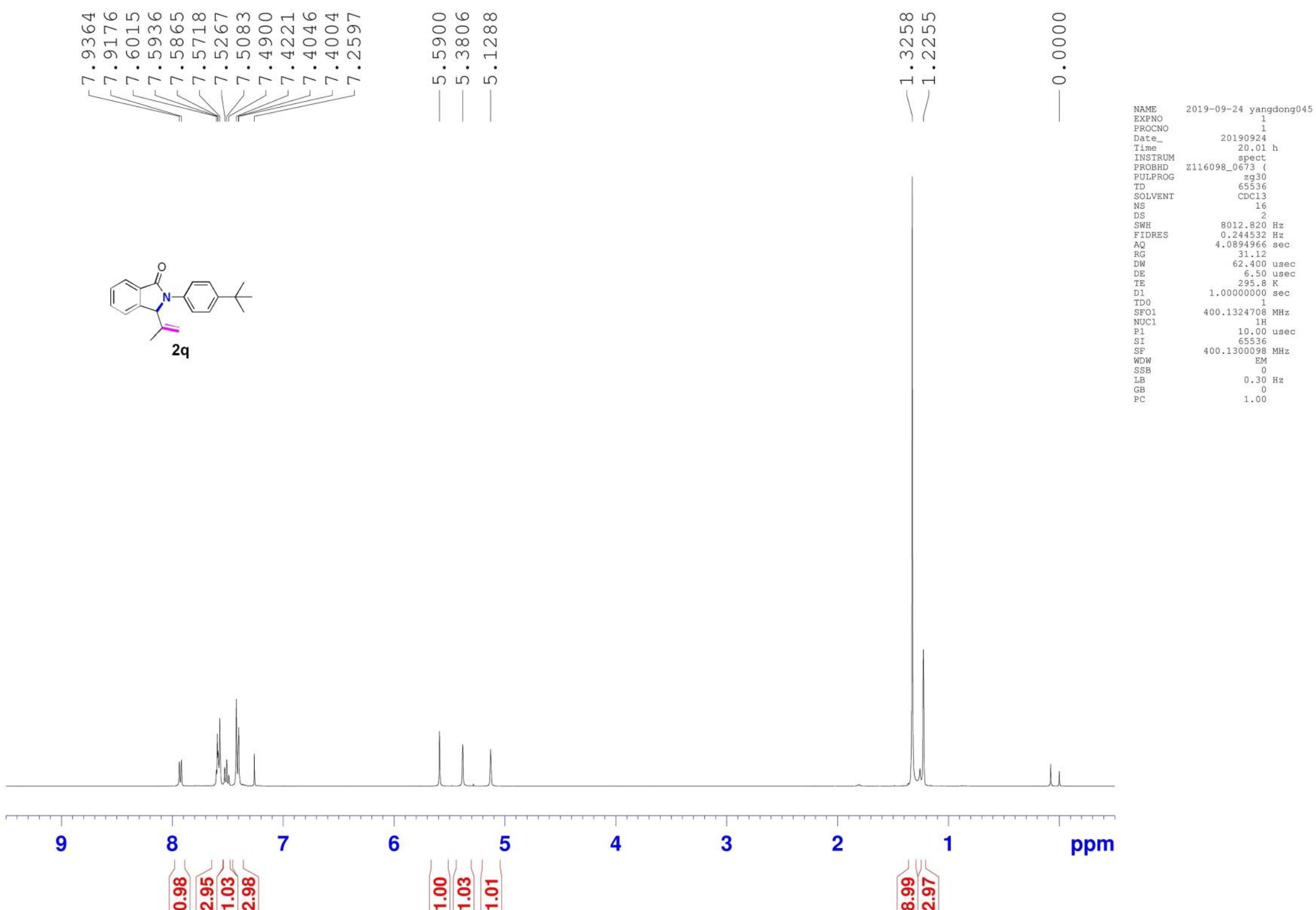

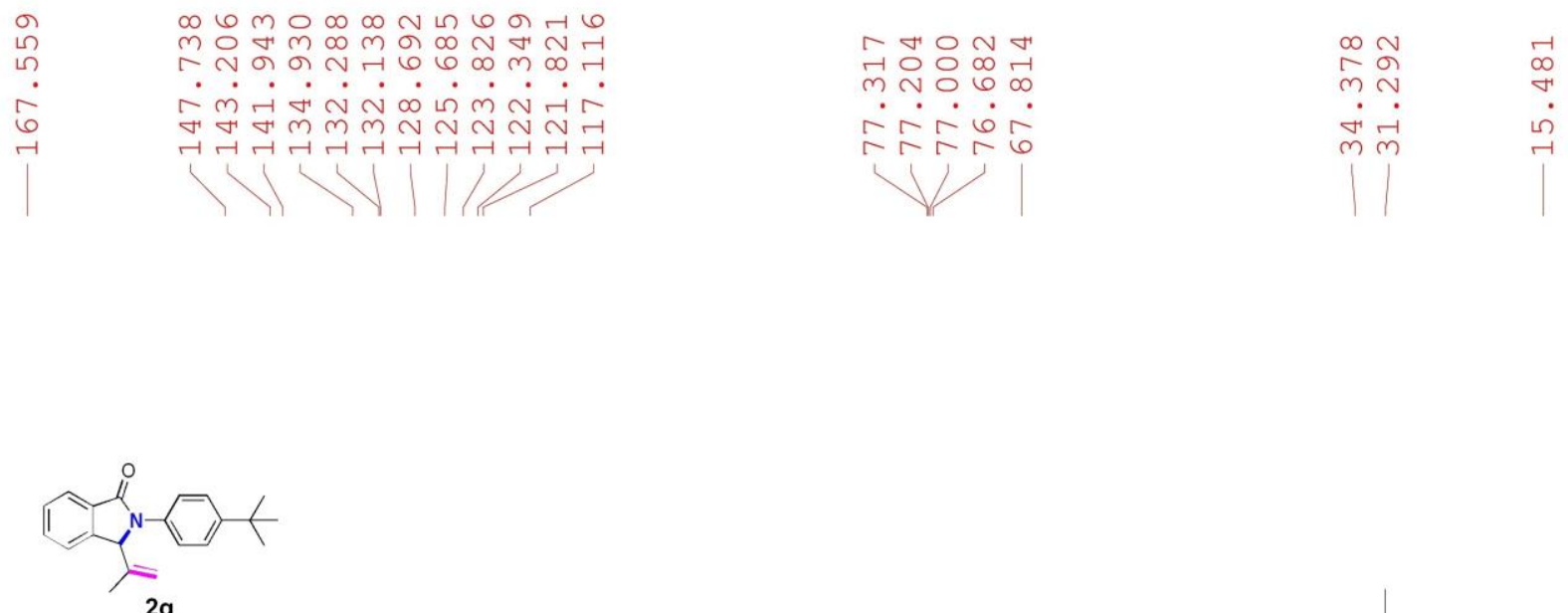

$2 q$
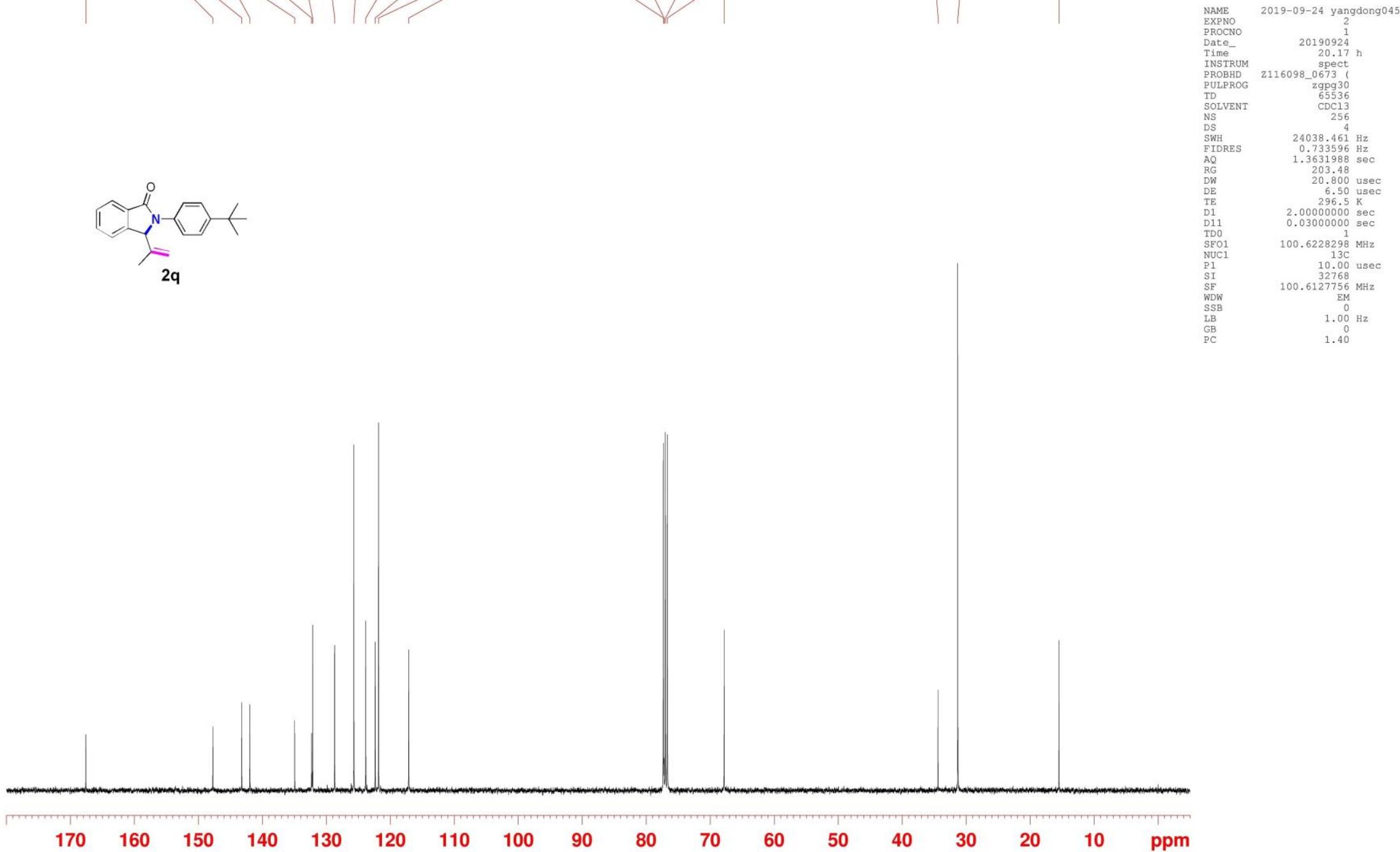


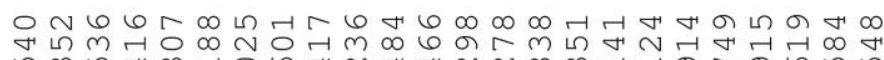
மூ

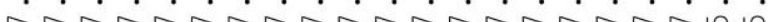

1
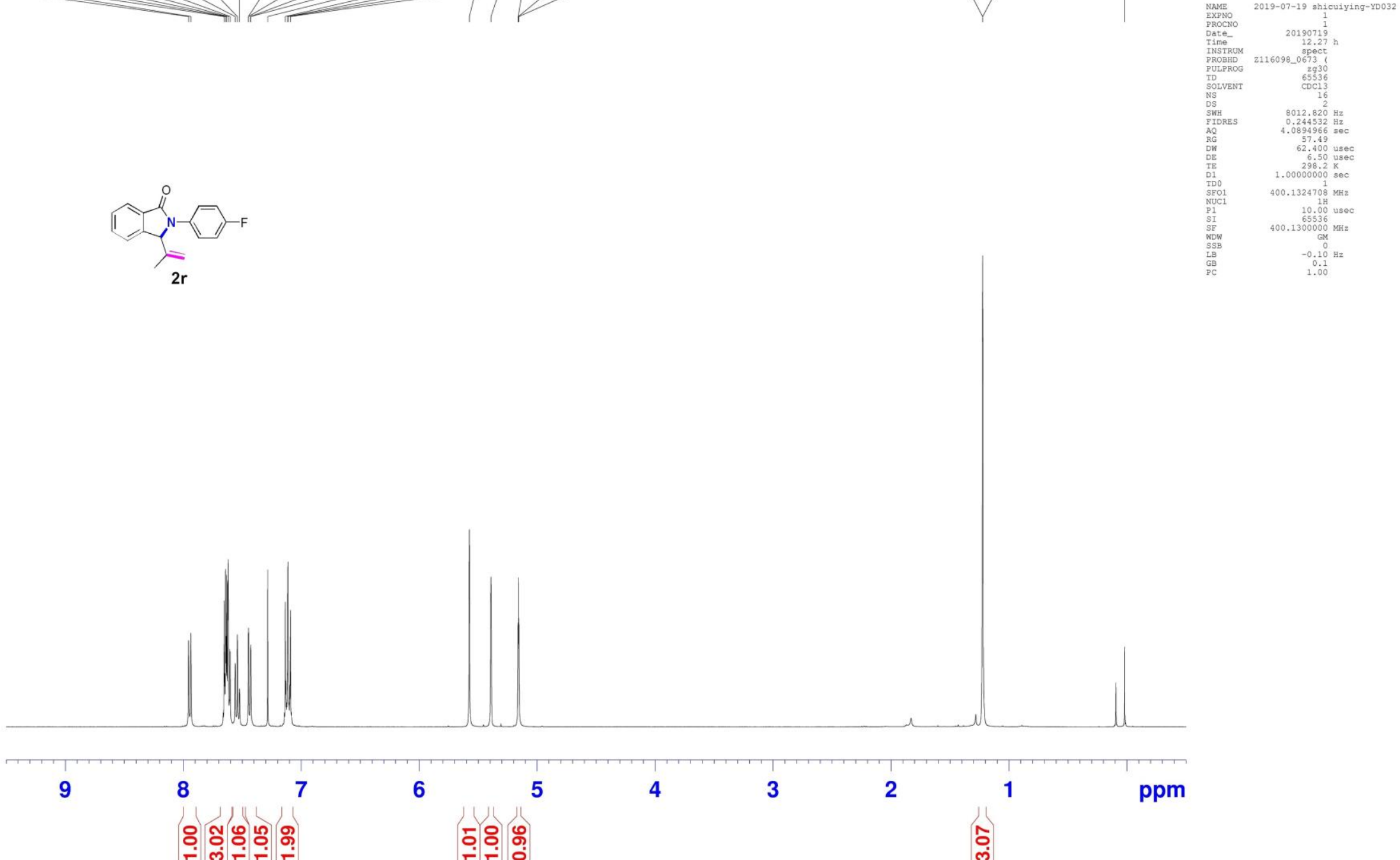


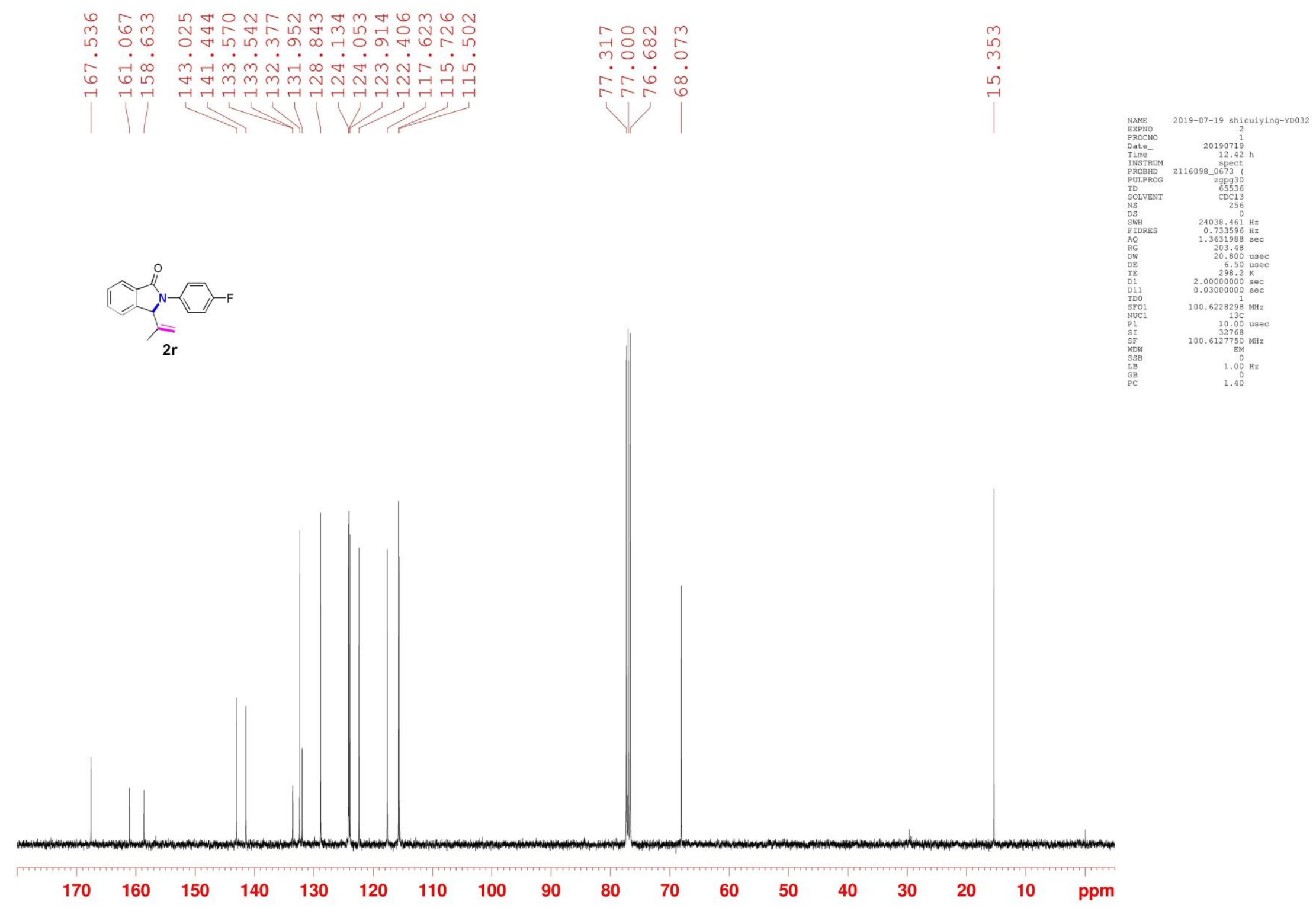




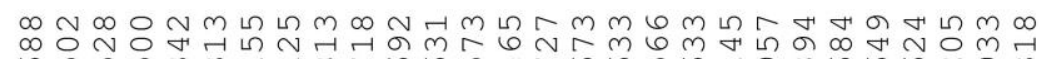

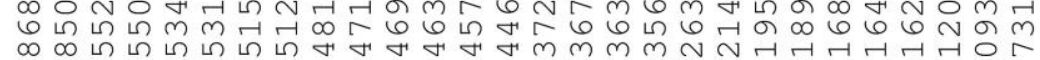
. . . n

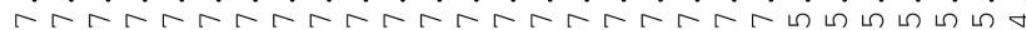
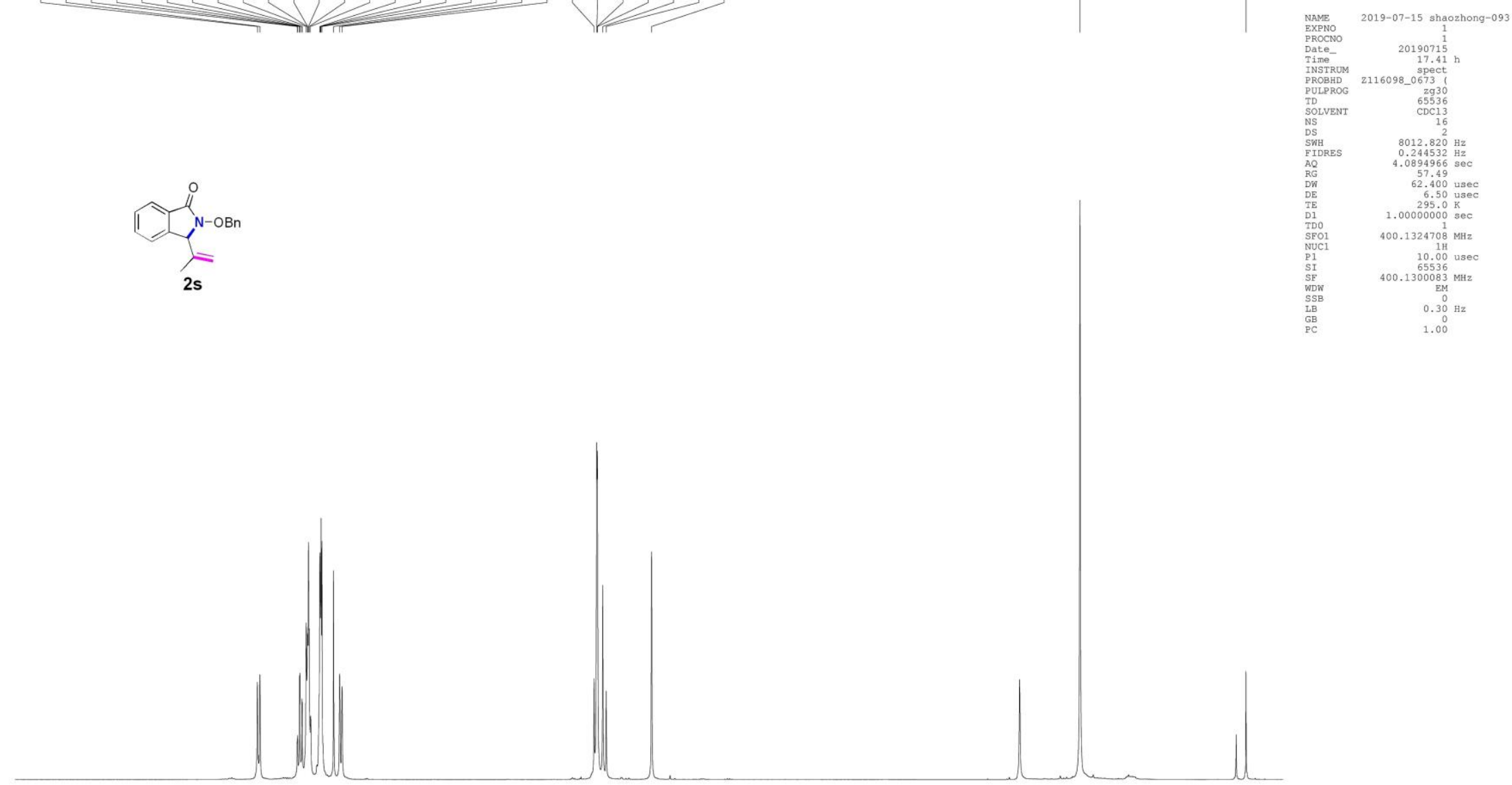

$\begin{array}{llllll}9.5 & 9.0 & 8.5 & 8.0 & 7.5 & 7.0\end{array}$

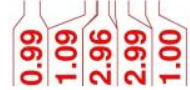
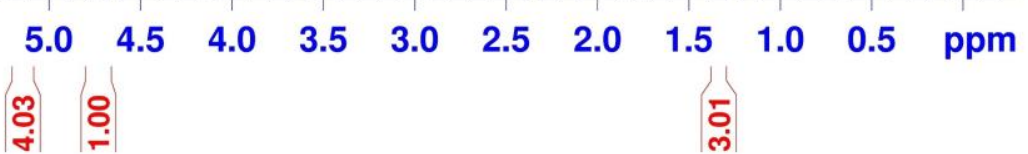


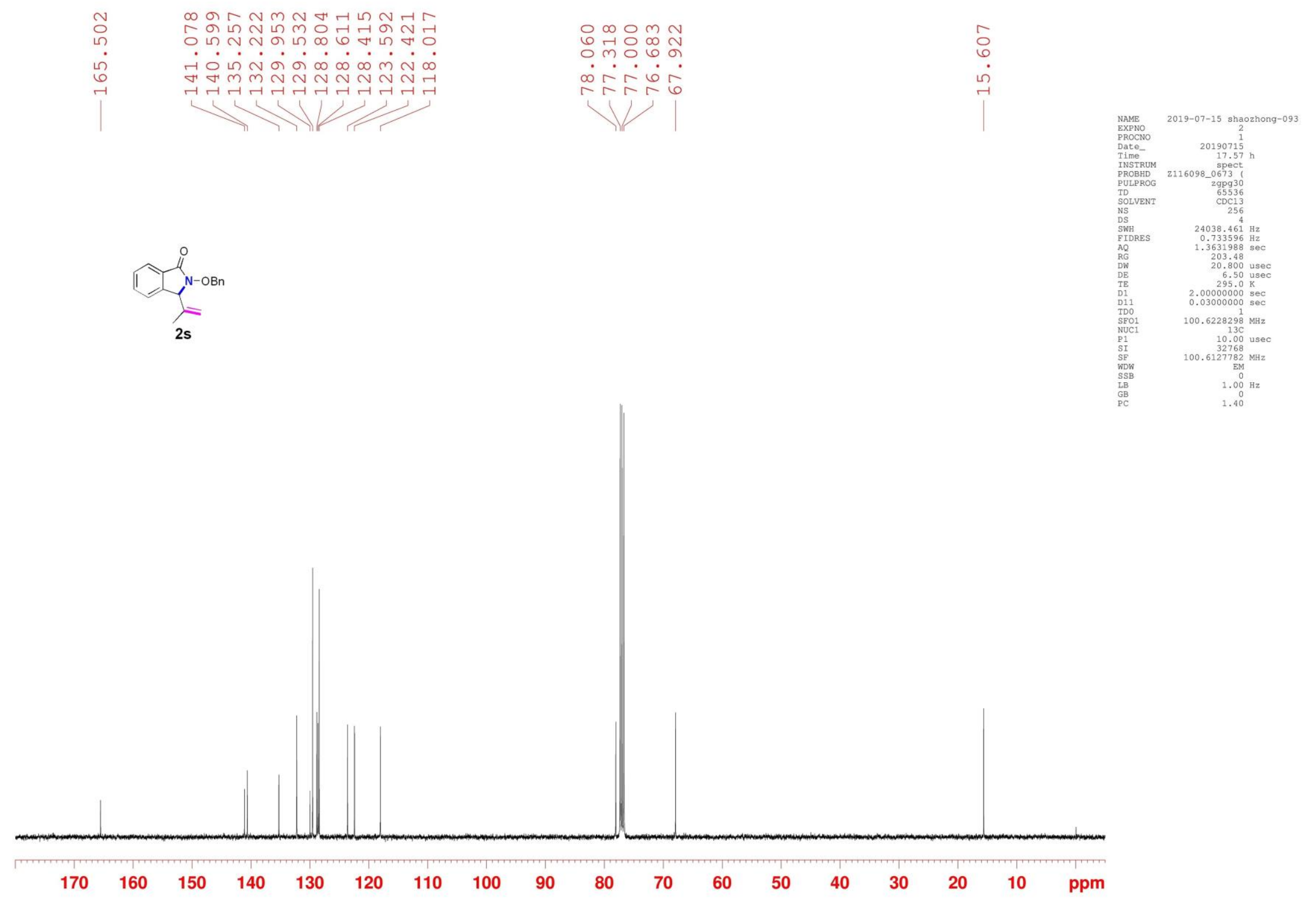


बㄱำ

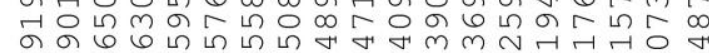

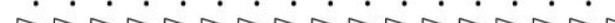

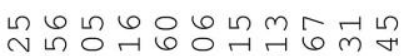

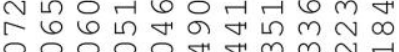
. $\operatorname{an}(4) 44-44-$
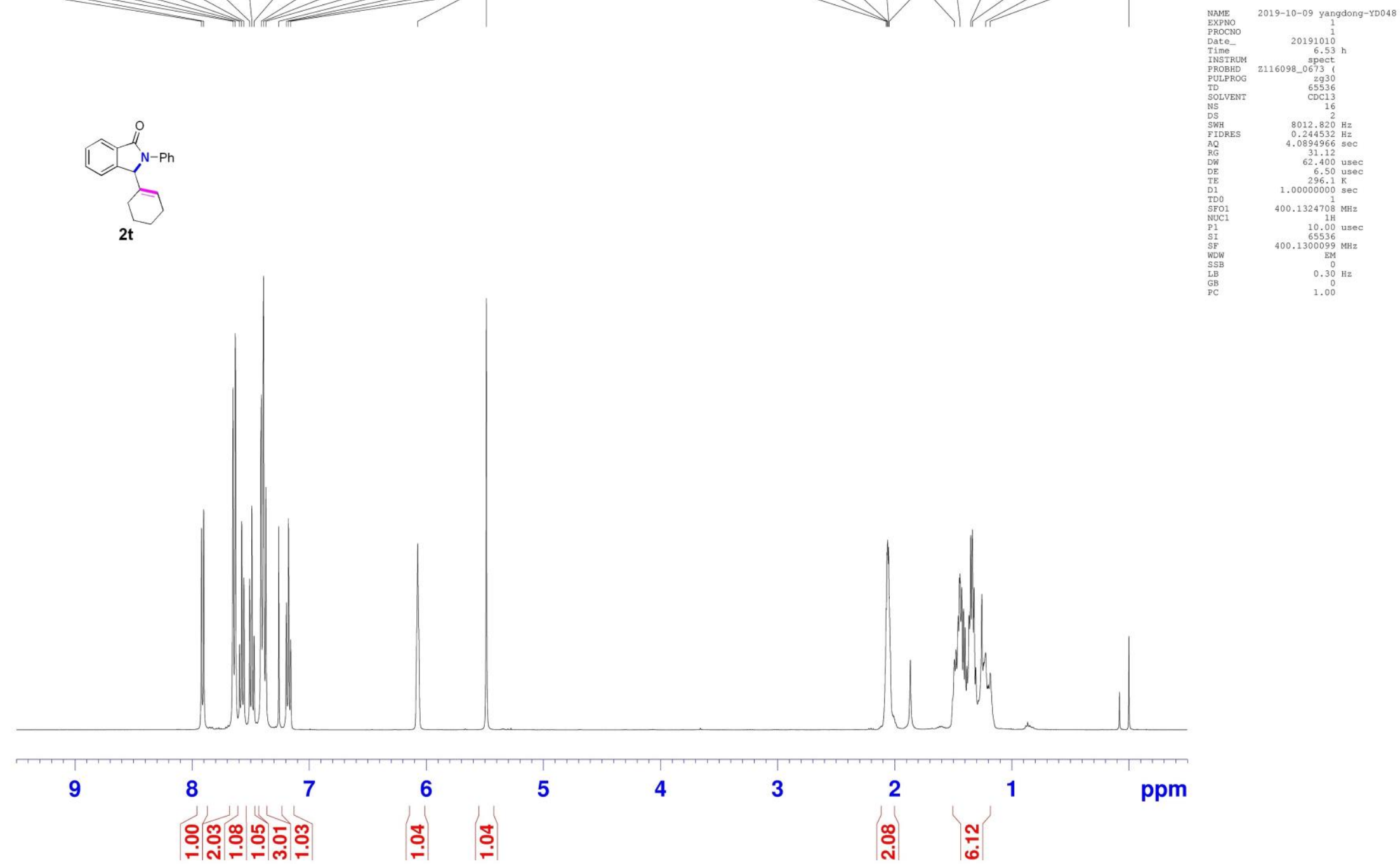


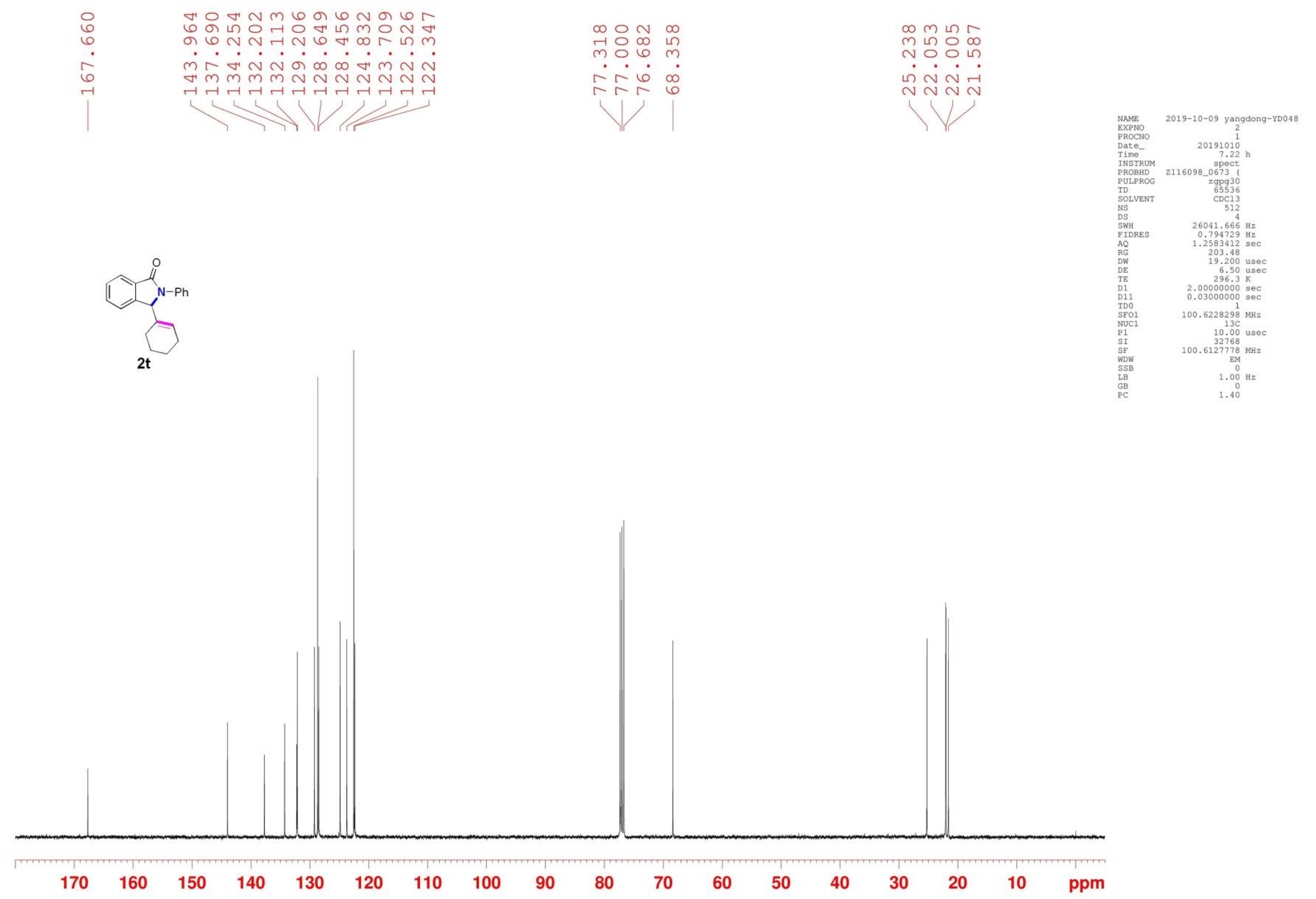




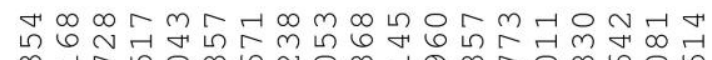

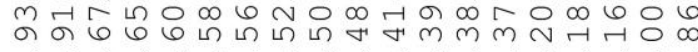

haratararataratarom

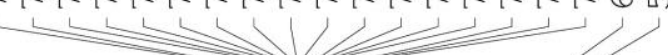

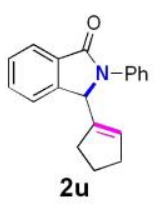

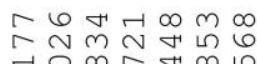

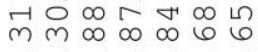

iथ

(1)
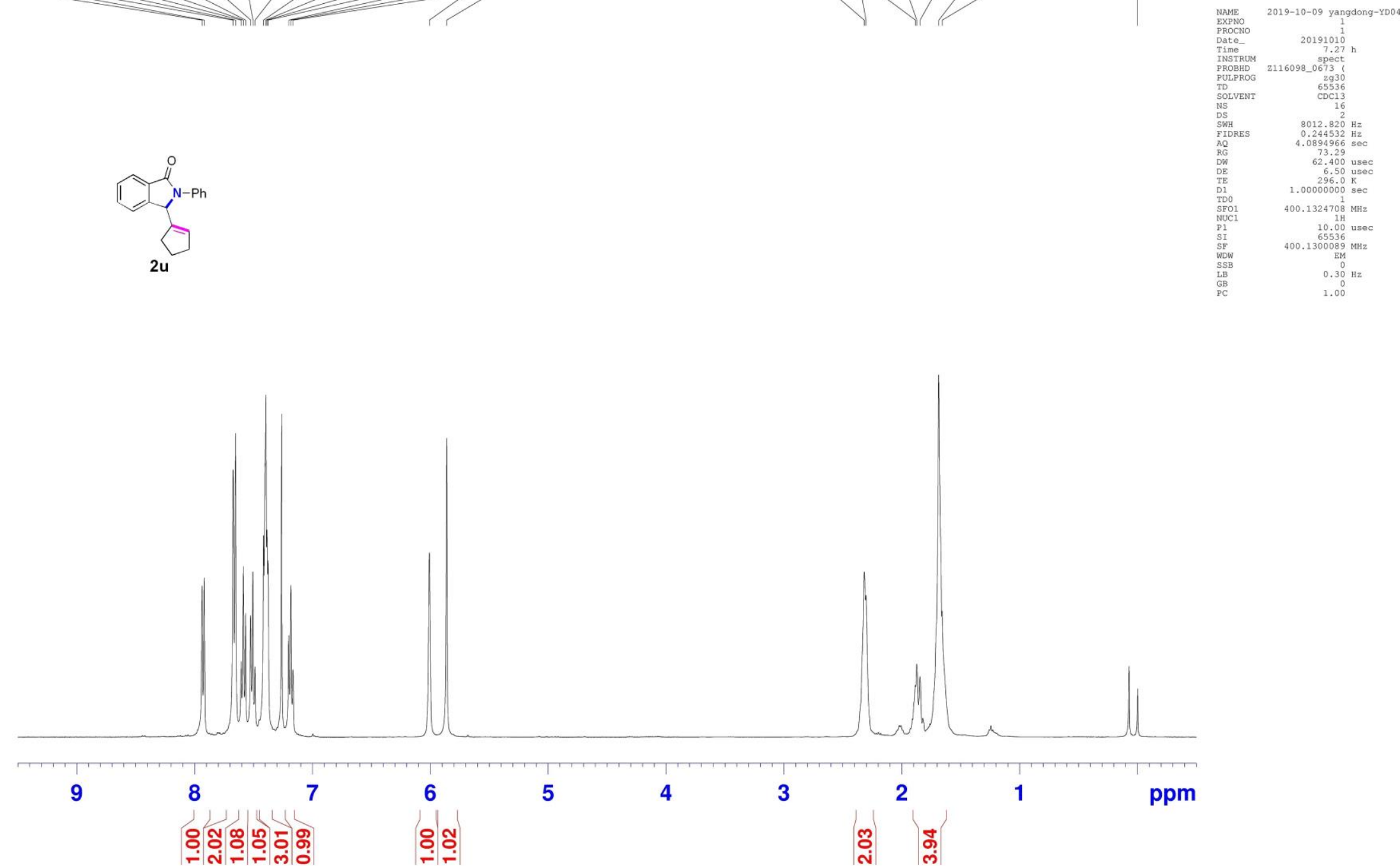


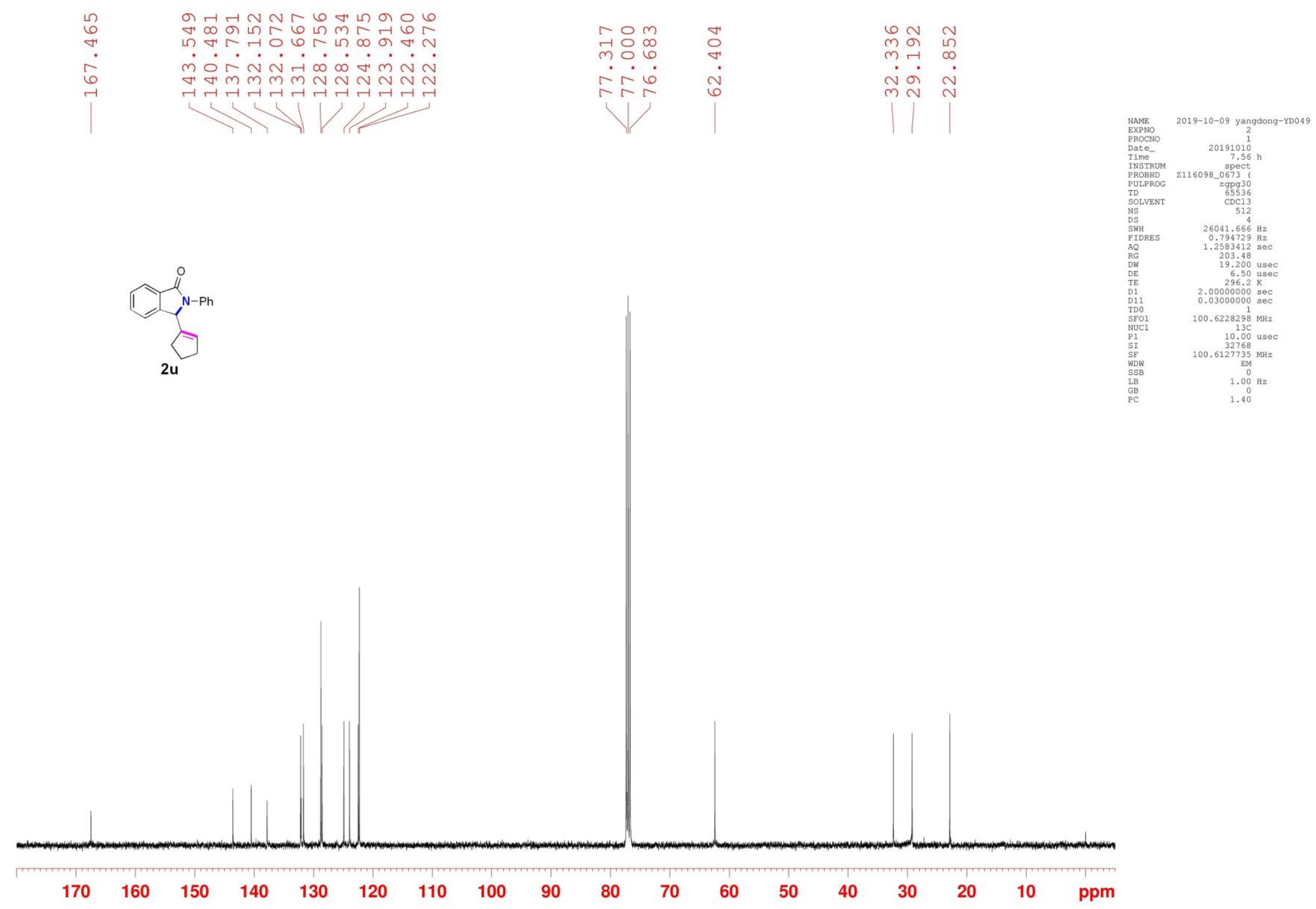




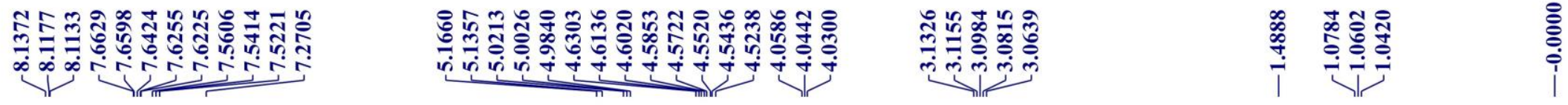

$\underbrace{\mathrm{NH}\left(\mathrm{SO}_{2} \mathrm{Ph}\right)_{2}}_{3}$

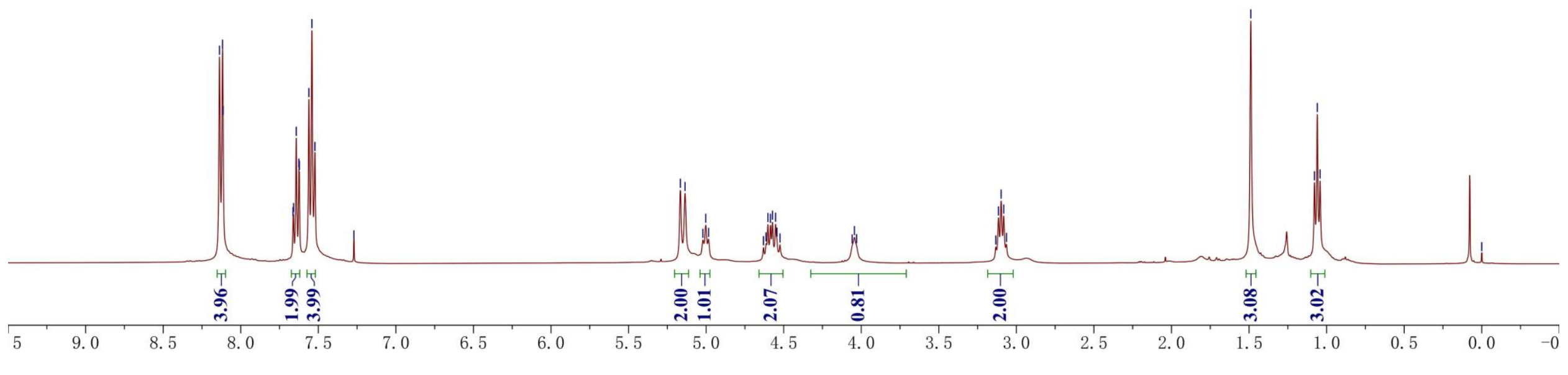




\begin{tabular}{|c|c|c|c|c|}
\hline 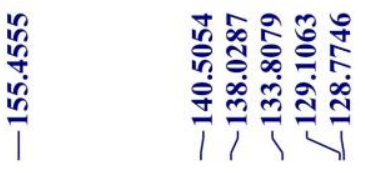 & 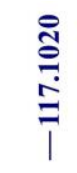 & 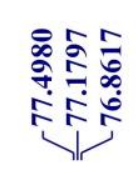 & 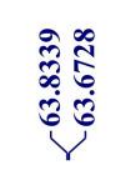 & 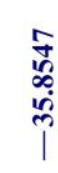 \\
\hline
\end{tabular}
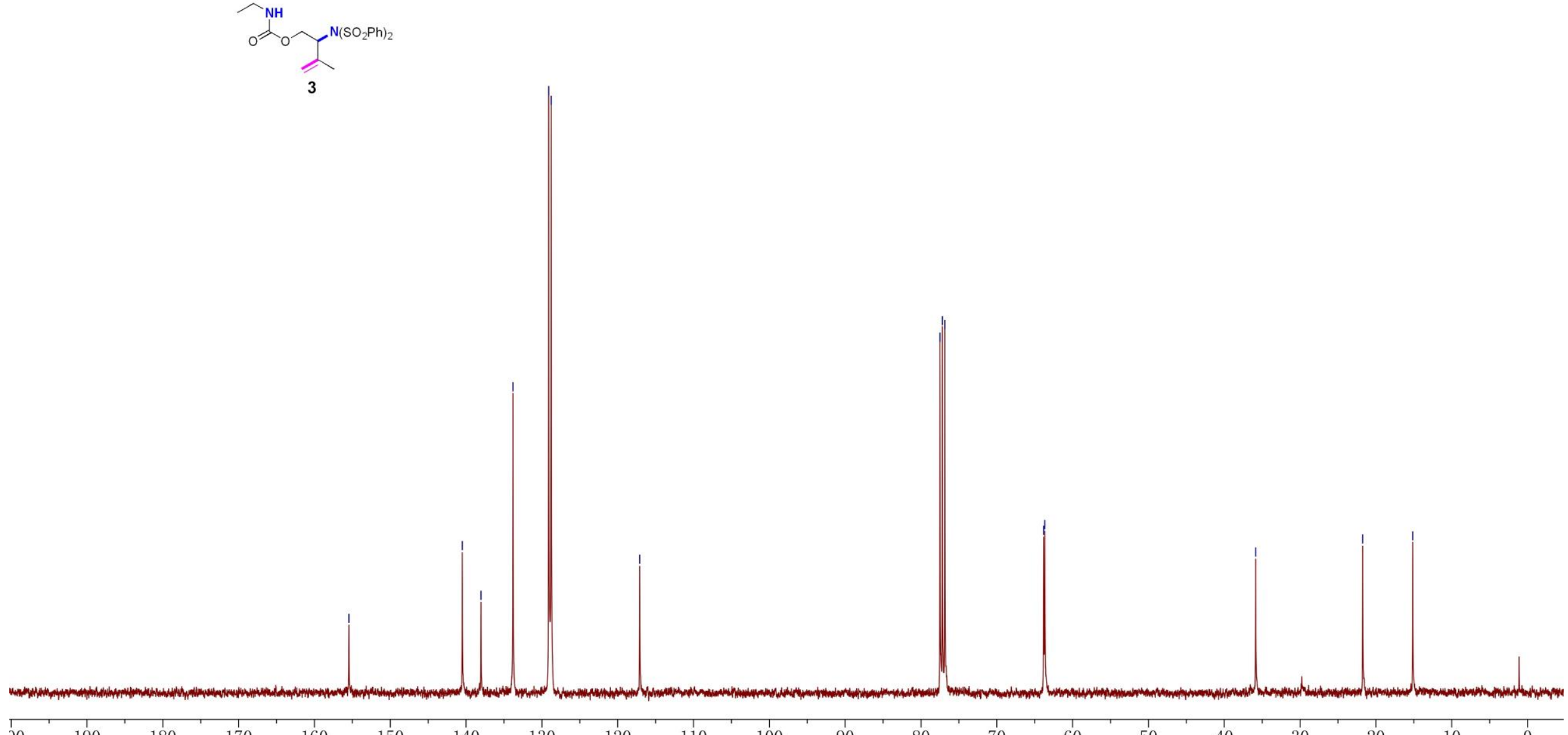


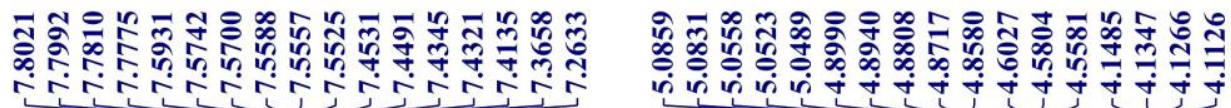
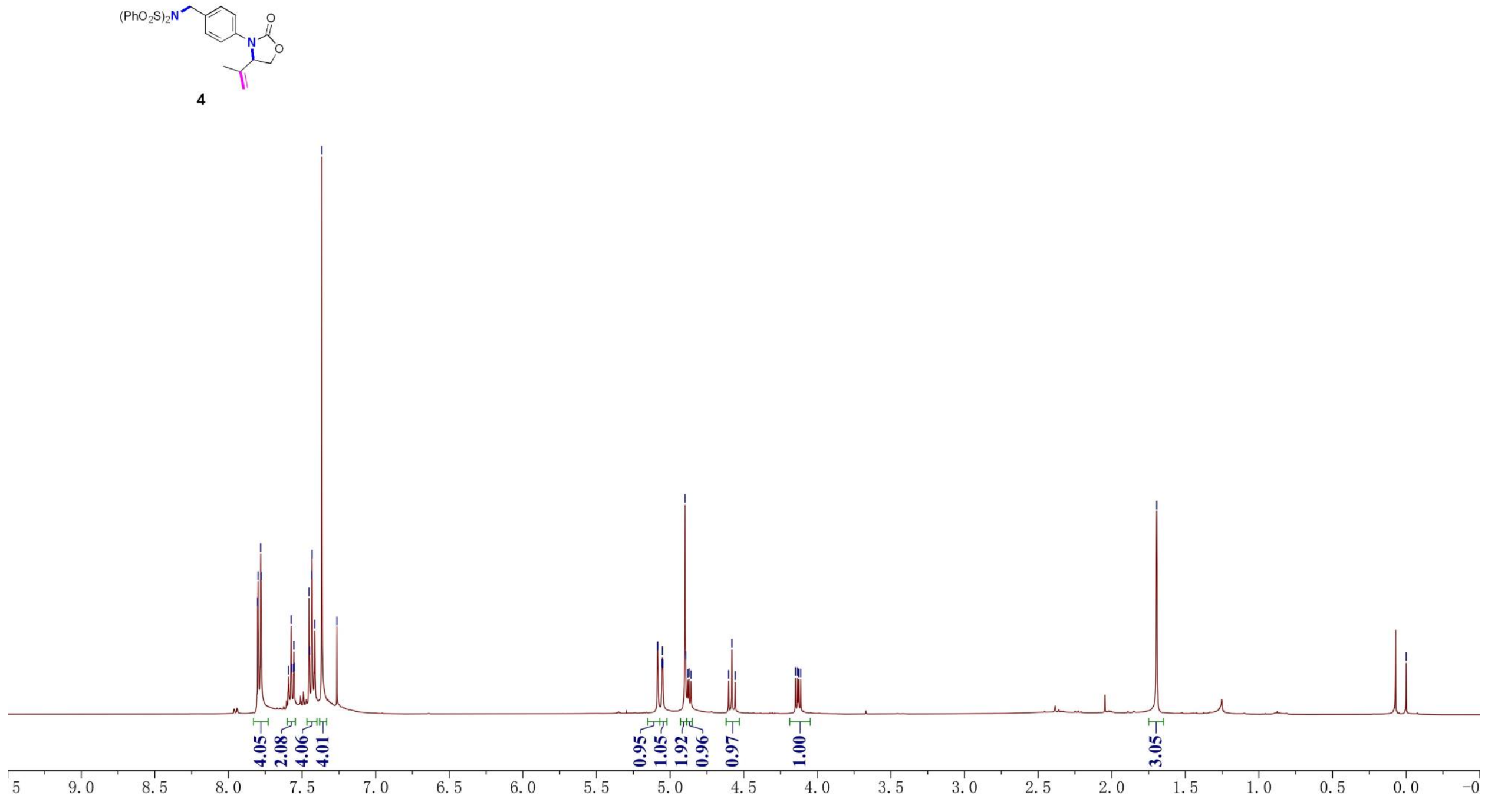


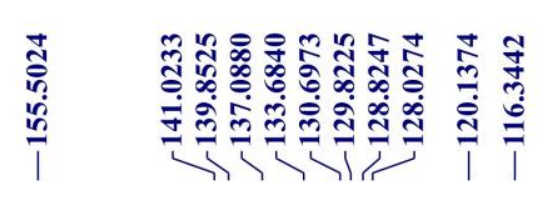

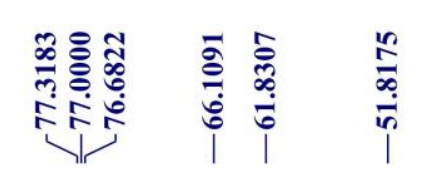

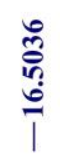

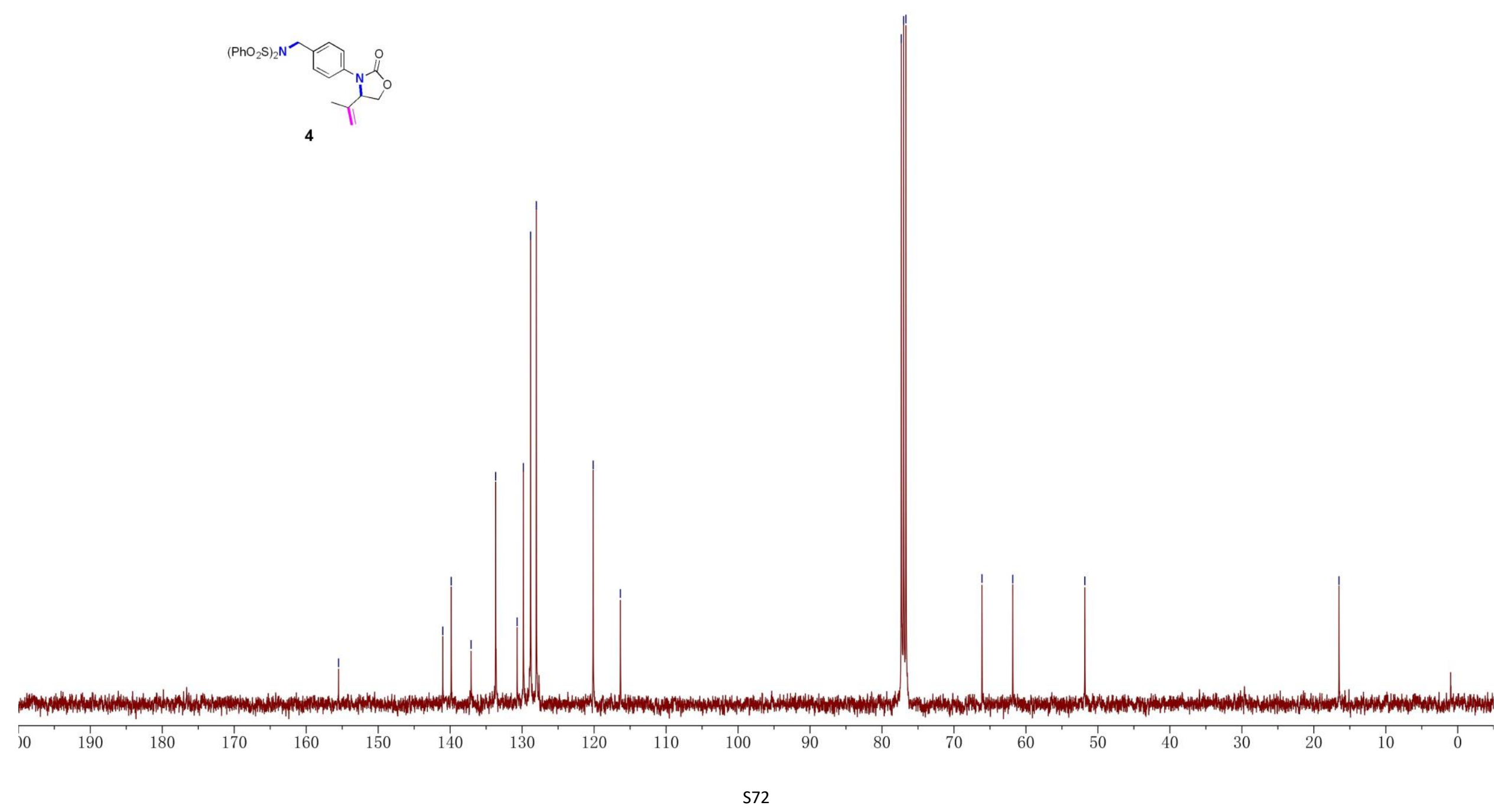



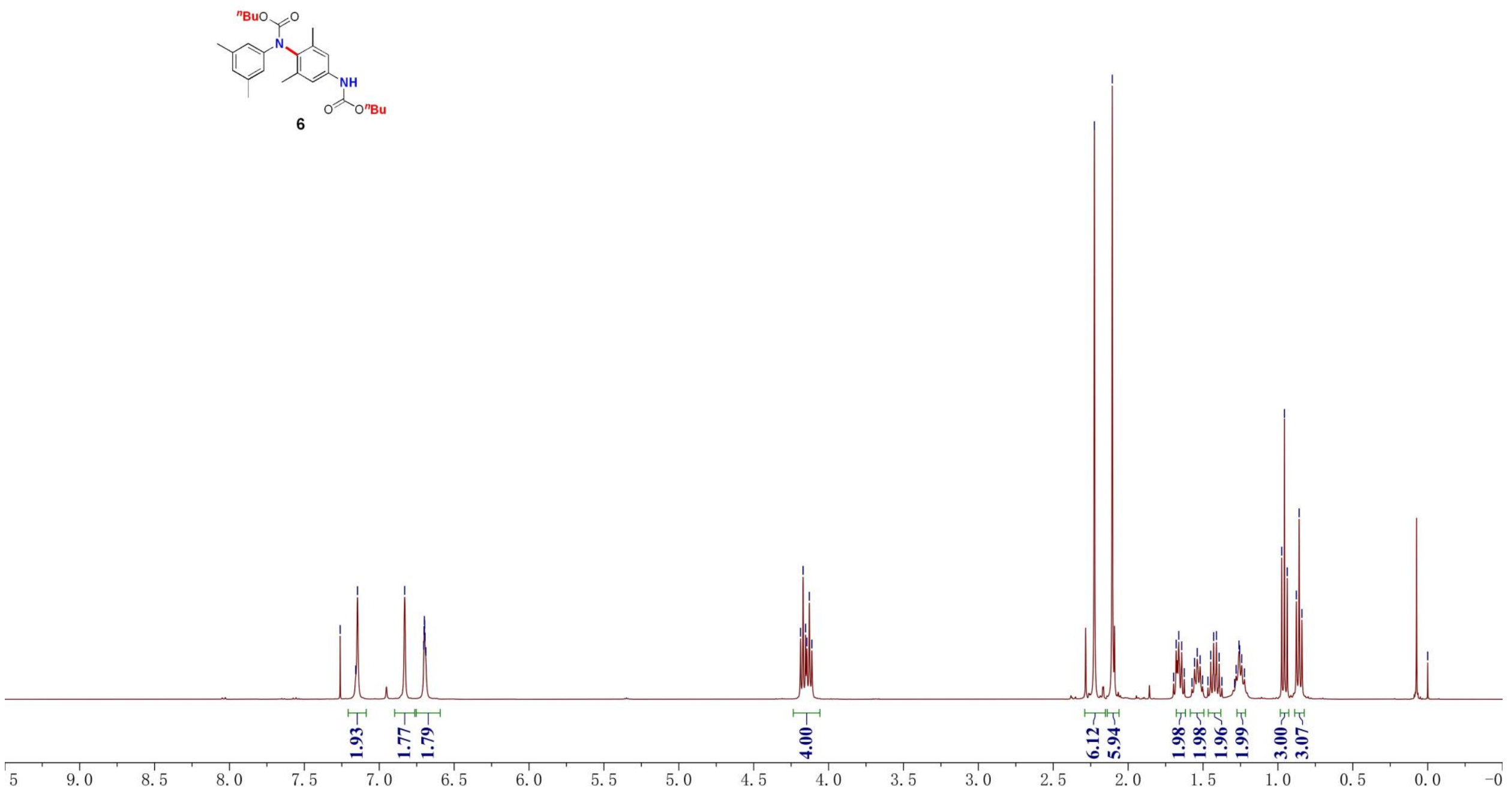

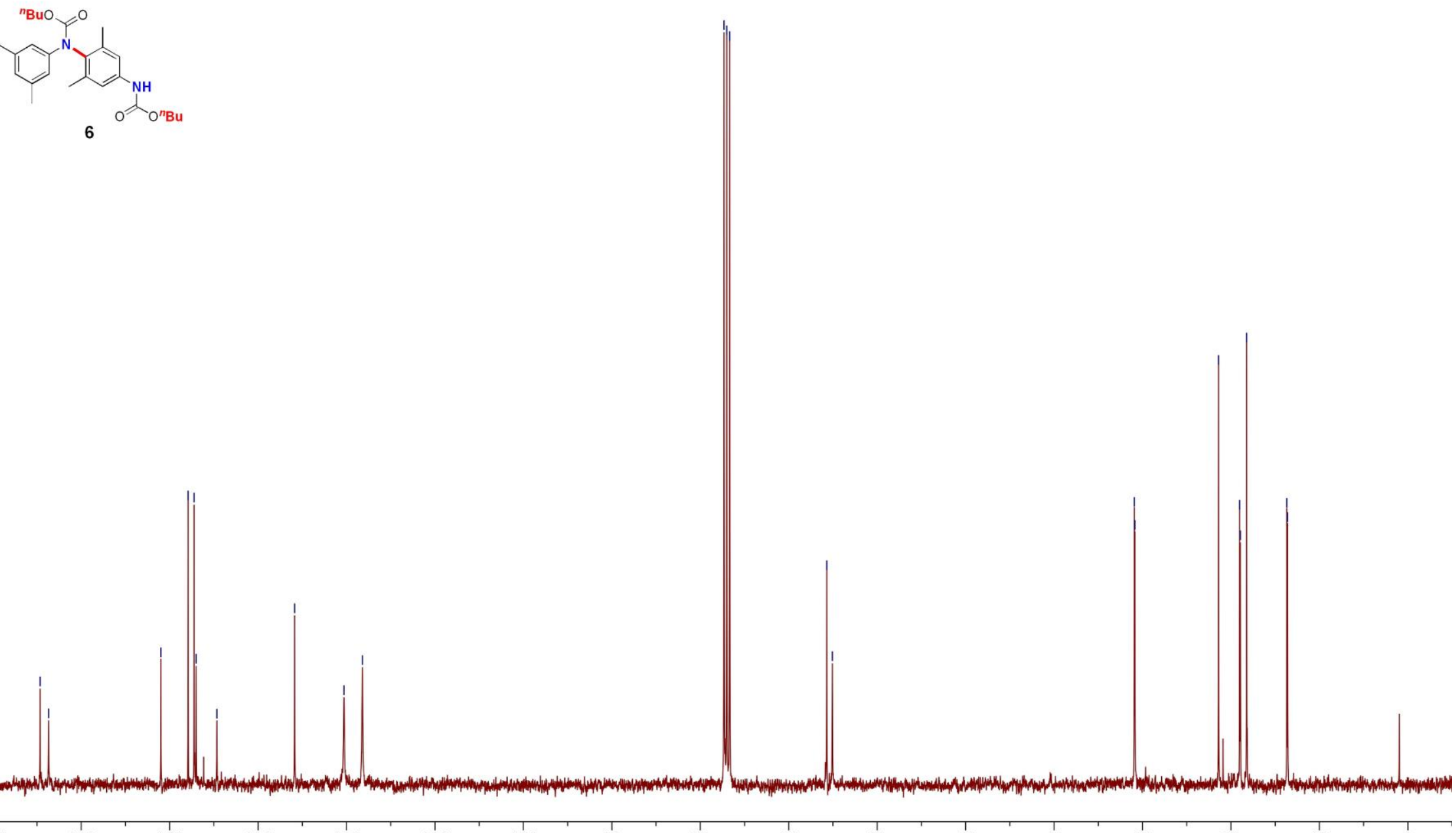

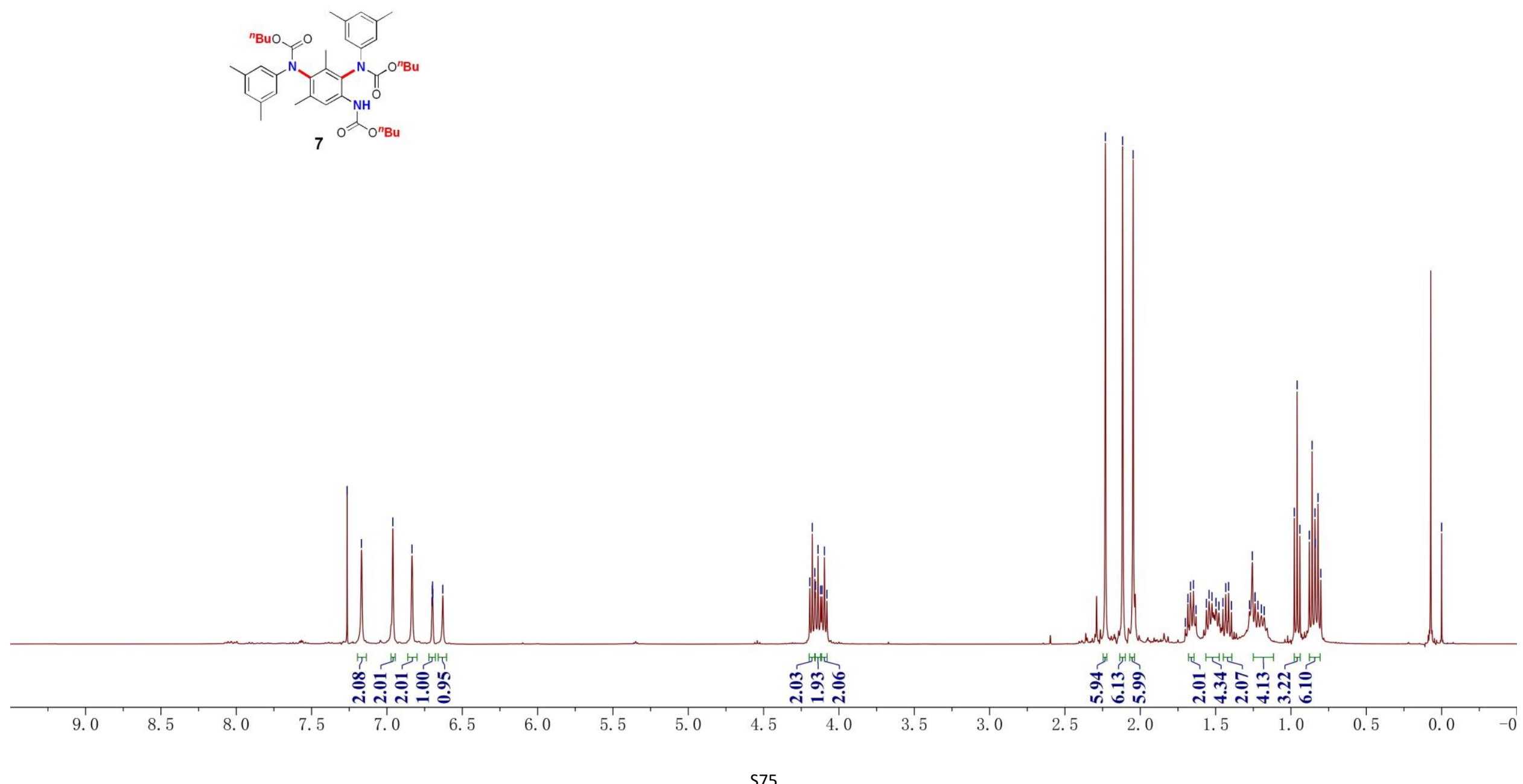

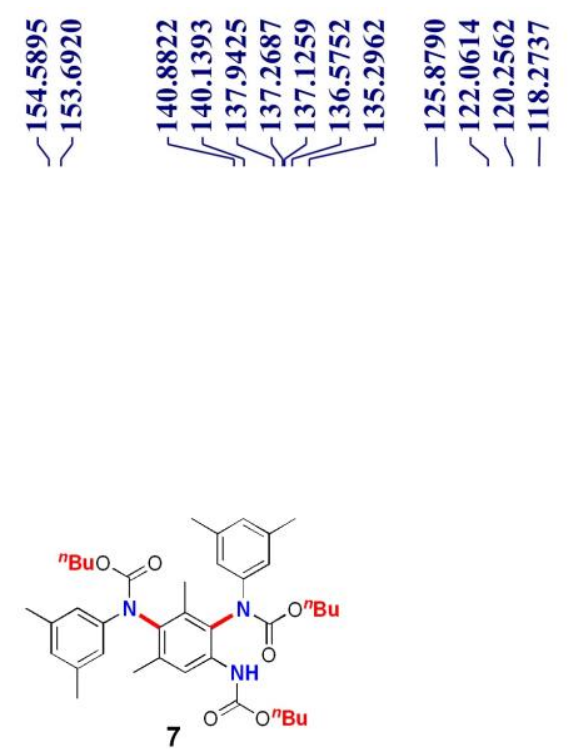


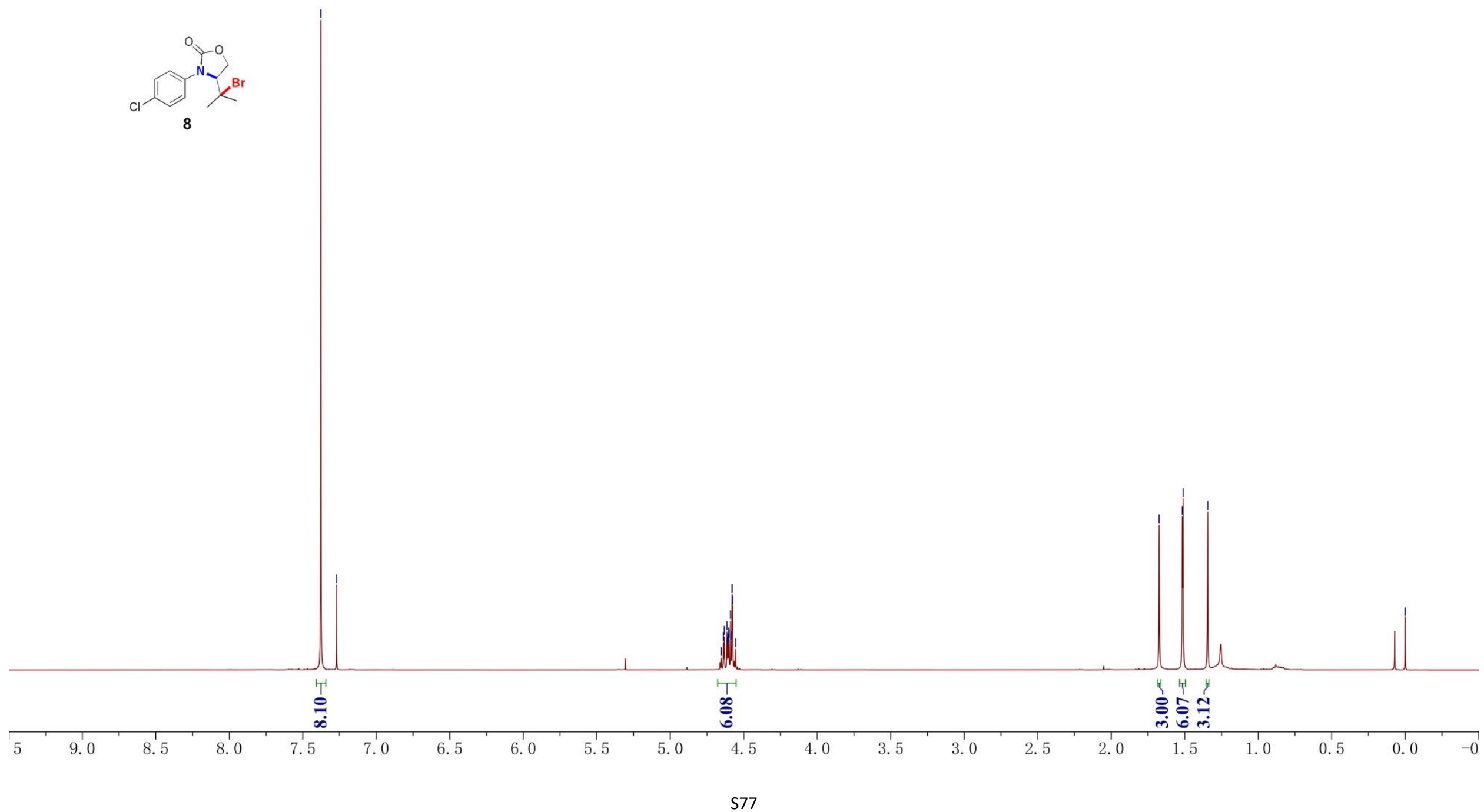



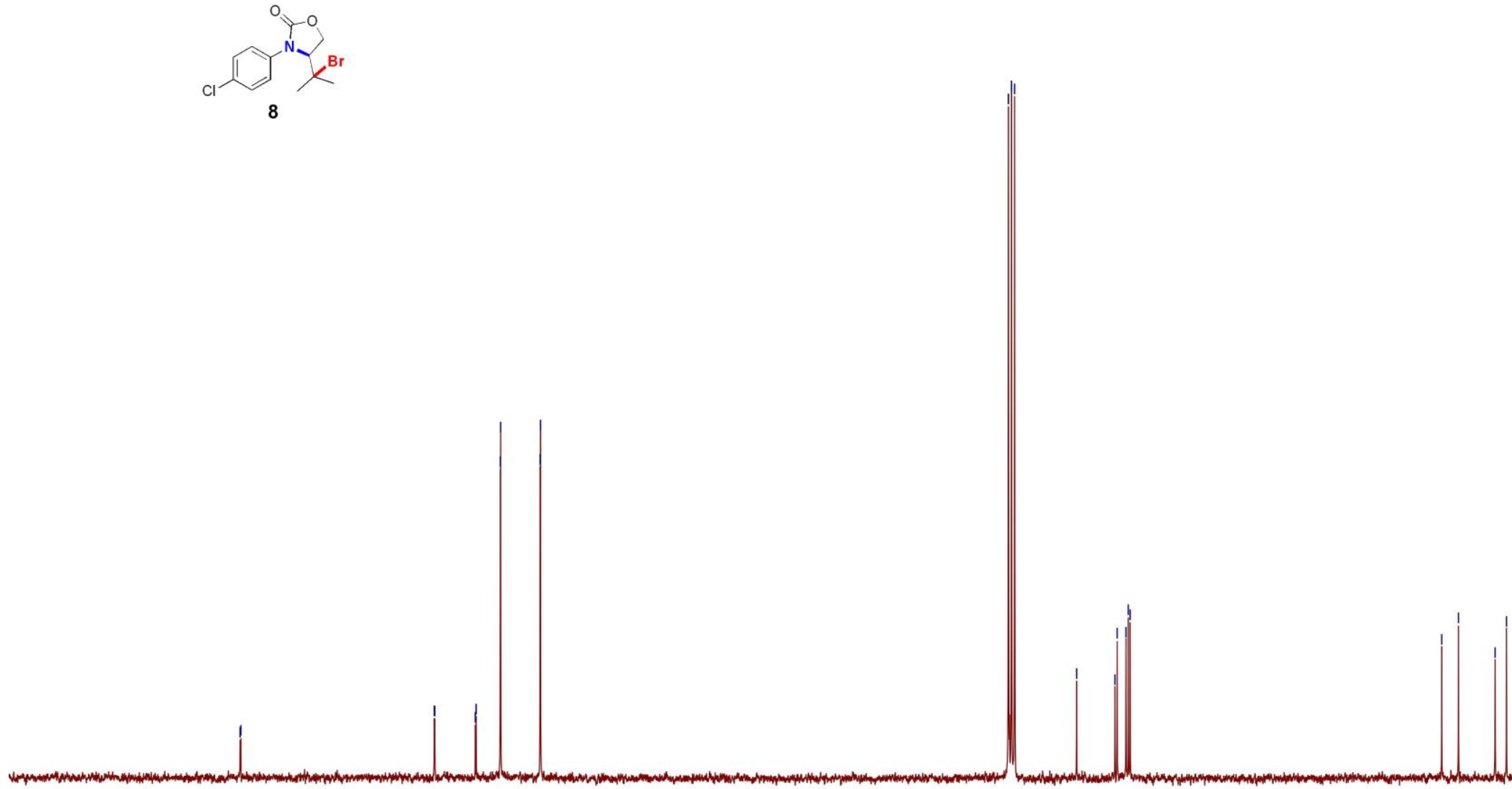